\title{
Positive Jantzen sum formulas for cyclotomic Hecke algebras
}

\author{
Andrew Mathas ${ }^{1}$
}

Received: 2 July 2021 / Accepted: 30 November 2021 / Published online: 2 March 2022

(c) Crown 2022, corrected publication 2022

\begin{abstract}
This paper proves a "positive" Jantzen sum formula for the Specht modules of the cyclotomic Hecke algebras of type $A$ and uses it to obtain new bounds on decomposition numbers. Quite remarkably, our results are proved entirely inside the cyclotomic Hecke algebras. Our positive sum formula shows that, in the Grothendieck group, the Jantzen sum formula can be written as an explicit non-negative linear combination of modules $\left[E_{f, e}^{\boldsymbol{v}}\right]$, which are the modular reductions of simple modules of related Hecke algebras in characteristic zero. The coefficient of $\left[E_{f, e}^{v}\right]$ in the sum formula is determined by the graded decomposition numbers in characteristic zero, which are known, and by the characteristic of the field. As a consequence we give an explicit upper bound for the decomposition numbers in characteristic $p>0$ in terms of linear combinations of decomposition numbers of a cyclotomic Hecke algebra at $e p^{r}$ th roots of unity in characteristic zero, for $r \geq 0$. Finally, we prove a new and more elegant "classical" Jantzen sum formula for these algebras.
\end{abstract}

Keywords Cyclotomic Hecke algebras · Graded cellular algebras · Khovanov Lauda Rouquier algebras

Mathematics Subject Classification 20G43 $\cdot 20 \mathrm{C} 08 \cdot 20 \mathrm{C} 30 \cdot 05 \mathrm{E} 10$

\section{Introduction}

Jantzen filtrations have played a dominant role in representation theory since they were introduced by Jantzen in 1977 [20]. In particular, stronger forms of the Lusztig and KazhdanLusztig conjectures [24,32] say that the Jantzen filtrations and weight filtrations of Weyl

Dedicated to Professor Gordon Douglas James, 31/12/1945-5/12/2020. This is the first paper that I have written about Jantzen sum formulas without my close collaborator and mentor Professor Gordon James. Gordon was a powerful and insightful mathematician who was a key player in shaping our current knowledge of the representation theory of the symmetric groups, Hecke algebras and Schur algebras. I feel privileged to have worked with him and I am proud to count him among my friends and colleagues. Even though Gordon did not contribute directly to this paper it is infused with his influence because it answers questions that Gordon and I discussed when we were working together.

\section{Andrew Mathas}

andrew.mathas@sydney.edu.au

1 School of Mathematics and Statistics F07, University of Sydney, Camperdown, NSW 2006, Australia 
modules coincide, results that are equivalent to (questions or) conjectures of Jantzen [21]. For quantum groups, complex semisimple Lie algebras, and Soergel bimodules these conjectures are now known to be true $[3,7,12,25,42,43]$.

Jantzen's observation for defining the Jantzen filtrations is extremely simple and elegant. Let $p$ be a rational prime and $M_{\mathbb{Z}}$ a finitely generated free $\mathbb{Z}$-module that comes equipped with a non-degenerate bilinear form $\langle,\rangle_{\mathbb{Z}}$. Then $M_{\mathbb{Z}}$ has a filtration $M_{\mathbb{Z}}=J_{0}\left(M_{\mathbb{Z}}\right) \supseteq J_{1}\left(M_{\mathbb{Z}}\right) \supseteq$ $J_{2}\left(M_{\mathbb{Z}}\right) \supseteq \cdots$ defined by

$$
J_{k}\left(M_{\mathbb{Z}}\right)=\left\{m \in M_{\mathbb{Z}} \mid\langle m, x\rangle \in p^{k} \mathbb{Z} \text { for all } x \in M_{\mathbb{Z}}\right\} .
$$

Reducing modulo $p$, let $M_{\mathbb{F}_{p}}=M \otimes_{\mathbb{Z}} \mathbb{F}_{p}$. The Jantzen filtration $M_{\mathbb{F}_{p}}=J_{0}\left(M_{\mathbb{F}_{p}}\right) \supseteq$ $J_{1}\left(M_{\mathbb{F}_{p}}\right) \supseteq J_{2}\left(M_{\mathbb{F}_{p}}\right) \supseteq \cdots$ of $M_{\mathbb{F}_{p}}$ is defined by:

$$
J_{k}\left(M_{\mathbb{F}_{p}}\right)=\left(J_{k}\left(M_{\mathbb{Z}}\right)+p M_{\mathbb{Z}}\right) / p M_{\mathbb{Z}}, \quad \text { for } k \geq 0 .
$$

Let $G_{M}$ be the Gram matrix of $\langle,\rangle_{\mathbb{Z}}$ with respect to some basis of $M_{\mathbb{Z}}$. By looking at the Smith normal form of $G_{M}$, Jantzen observed the trivial but important fact that

$$
v_{p}\left(\operatorname{deg} G_{M}\right)=\sum_{k>0} \operatorname{dim}_{\mathbb{F}_{p}} J_{k}\left(M_{\mathbb{F}_{p}}\right),
$$

where $v_{p}$ is the usual $p$-adic valuation. In category $\mathscr{O}$, a Weyl module $\Delta^{\lambda}$ is uniquely determined by the dimensions of its weight spaces. Applying this machinery to the weight spaces $\Delta_{\mu}^{\Lambda}$ of $\Delta^{\lambda}$ determines the dimensions of the $\mu$-weight spaces in the Jantzen filtration of a Weyl module $\Delta^{\lambda}$. This leads to the Jantzen sum formula of $\Delta^{\lambda}$, which describes the $\operatorname{sum} \sum_{k>0}\left[J_{k}\left(\Delta^{\lambda}\right)\right]$ in the Grothendieck group as an explicit $\mathbb{Z}$-linear combination of more dominant Weyl module [22, II.8.19].

One limitation of all of the Jantzen sum formulas in the literature is that they express $\sum_{k>0}\left[J_{k}(M)\right]$ in the Grothendieck group as a $\mathbb{Z}$-linear combination of more dominant Weyl modules or Specht modules, where many of the coefficients are negative. This is "wrong" in the sense that $\sum_{k>0}\left[J_{k}(M)\right]$ can definitely be written as a non-negative linear combination of the images of the simple modules in the Grothendieck group. On the other hand, these negative coefficients in the sum formulas have to appear because, in general, the simple modules cannot be written as non-negative linear combinations of the Weyl modules in the Grothendieck group. It is natural to ask for a new version of the Jantzen sum formula that expresses $\sum_{k>0}\left[J_{k}(M)\right]$ as a non-negative linear combination of simple modules.

Soon after Jantzen proved his sum formula, his student Schaper used Jantzen's sum formula for the general linear groups to prove a sum formula for the Specht modules of the symmetric groups [40, Satz 4]. Analogues of this result have since been proved for the Specht modules of the Ariki-Koike algebras $[18,19]$, which are cyclotomic Hecke algebras of type $A$ and which include the symmetric group and their Iwahori-Hecke algebras as special cases. In the literature, rather than working in the module category of the Hecke algebras, this result is always deduced by first proving a sum formula the Weyl modules of a related Schur algebra and then applying a Schur functor to deduce the sum formula for the Specht modules. In particular, the literature does not contain the proof of a sum formula for these algebras that takes place entirely inside the module category of the Ariki-Koike algebras or the symmetric groups.

This paper proves a new positive Jantzen sum formula for the Specht modules of the cyclotomic Hecke algebras of type $A$ that, in the Grothendieck group, writes $\sum_{k>0}\left[J_{k}\left(S^{\lambda}\right)\right]$ as an explicit non-negative linear combination of the images of certain modules $E_{f, e}^{\mu}$. This sum formula is proved entirely within the module categories of the Hecke algebras. The 
modules $E_{f, e}^{\mu}$ that appear in our sum formula are not, in general, simple. Instead these modules are modular reductions of the simple modules of related cyclotomic Hecke algebras in characteristic zero. The coefficients that appear in the positive sum formula are described explicitly in terms of (the derivatives of graded) decomposition numbers.

To state our main result we quickly introduce the notation that we need and refer the reader to later sections for the full definitions. Let $F$ be a field of characteristic $p \geq 0$ and let $\mathscr{H}_{n}^{\Lambda}(F)$ be a cyclotomic Hecke algebra over $F$ with a Hecke parameter $\xi$ of quantum characteristic $e$ (Definition 3.2). Let $\mathcal{P}_{n}^{\Lambda}$ be the set of $\ell$-partitions. For $\lambda \in \mathcal{P}_{n}^{\Lambda}$ let $S_{F}^{\lambda}$ be the corresponding Specht module for $\mathscr{H}_{n}^{\Lambda}(F)$. If $M$ is an $\mathscr{H}_{n}^{\Lambda}(F)$-module let $[M]$ be its image in the Grothendieck group $\operatorname{Rep} \mathscr{H}_{n}^{\Lambda}(F)$ of $\mathscr{H}_{n}^{\Lambda}(F)$.

For $f=e p^{r}$, for $r \geq 0$, let $\zeta_{f} \in \mathbb{C}$ be a primitive $f$ th root of unity and let $\left\{E_{\mathbb{C}, f}^{\boldsymbol{v}} \mid \boldsymbol{v} \in \mathcal{K}_{n}^{\Lambda_{f}}\right\}$ be the set of simple modules for a corresponding Hecke algebra $\mathscr{H}_{n}^{\Lambda_{f}}(\mathbb{C})$ over $\mathbb{C}$ that has Hecke parameter $\zeta_{f}$; see (4.17). By Proposition 4.18, there is a map of Grothendieck groups $\mathrm{A}_{f, e}: \operatorname{Rep} \mathscr{H}_{n}^{\Lambda_{f}}(\mathbb{C}) \longrightarrow \operatorname{Rep} \mathscr{H}_{n}^{\Lambda}(F)$ when $p>0$. Let $E_{f, e}^{v}=\mathrm{A}_{f, e}\left(E_{\mathbb{C}, f}^{v}\right)$ and set $E_{f, e}^{v}=0$ if $p=0$. Finally, let $d_{\lambda \mu}^{\mathbb{C}, f}(q)$ be a graded decomposition number for $\mathscr{H}_{n}^{\Lambda_{f}}(\mathbb{C})$, for $\lambda \in \mathcal{P}_{n}^{\Lambda}$ and $\boldsymbol{\mu} \in \mathcal{K}_{n}^{\Lambda_{f}}$; see (2.15). Then $d_{\lambda \mu}^{\mathbb{C}, f}(q)$ is a parabolic Kazhdan-Lusztig polynomial that, in principle, is known. Let $\left(d_{\lambda \mu}^{\mathbb{C}, f}\right)^{\prime}(1)$ be the derivative of $d_{\lambda \mu}^{\mathbb{C}, f}(q)$ evaluated at $q=1$. Our main result is then the following:

Main Theorem Suppose that $F$ is a field of characteristic $p \geq 0$ and let $\lambda \in \mathcal{P}_{n}^{\Lambda}$. In $\operatorname{Rep} \mathscr{H}_{n}^{\Lambda}(F)$,

$$
\sum_{k>0}\left[J_{k}\left(S_{F}^{\lambda}\right)\right]=\sum_{\boldsymbol{\mu} \in \mathcal{K}_{n}^{\Lambda}}\left(d_{\lambda \mu}^{\mathbb{C}, e}\right)^{\prime}(1)\left[E_{F, e}^{\mu}\right]+\sum_{f=e p^{r}>e}(p-1) p^{r-1} \sum_{\boldsymbol{v} \in \mathcal{K}_{n}^{\Lambda_{f}}}\left(d_{\lambda \boldsymbol{v}}^{\mathbb{C}, f}\right)^{\prime}(1)\left[E_{f, e}^{\boldsymbol{v}}\right] .
$$

Strikingly, in characteristic $p$ the right hand side of this sum formula depends on modules for Hecke algebras at $e p^{r}$ th roots of unity. Moreover, by Lemma 4.13 below, all of the coefficients on the right hand side of the sum formula are non-negative integers, so this really is a positive Jantzen sum formula.

When $F$ is a field of characteristic zero the second sum over $r>0$ on the right hand side of our main theorem vanishes. In this case, the new sum formula can be proved by assuming a deep conjecture which says that the Jantzen filtrations of graded Specht modules coincide with their grading filtrations. We cannot prove our main result this way because the Jantzen filtrations are only known to coincide with the grading filtrations for cyclotomic Hecke algebras of level 1, where this follows from work of Shan [41, Theorem 0.1]. (Shan actually proves that the Jantzen filtrations of the Weyl modules of the $q$-Schur algebras coincide with their grading filtrations. As the graded Schur functor maps the Jantzen filtration of a Weyl module to the Jantzen filtration of the corresponding Specht module, and sends grading filtrations to grading filtrations, this implies that if $\Lambda=\Lambda_{0}$ then the Jantzen and grading filtrations of the Specht modules of $\mathscr{H}_{n}^{\Lambda}(\mathbb{C})$ coincide.)

The proof of our main theorem takes place entirely inside the module categories of the cyclotomic Hecke algebras. The main idea is to work with the formal characters of $\mathscr{H}_{n}^{\Lambda}(F)$ modules (Definition 3.15). The key point is that character map is injective so the images of all modules in the Grothendieck group, including the images of the Jantzen filtration, are determined by their characters. This is essentially the same idea sketched above for computing the Jantzen sum formula of Weyl modules

Using seminormal forms it is relatively easy to determine the "Jantzen characters" (Proposition 4.3). The proof of our main theorem follows by applying some combinatorial tricks 
using the formal characters of the graded Specht modules of the cyclotomic KLR algebras type $A$, which are isomorphic to the cyclotomic Hecke algebras. It is quite remarkable that the graded Specht modules enter this story, but their appearance in our arguments is almost accidental because instead of marking a direct connection between the Jantzen filtrations and the graded representation theory we only exploit some combinatorial shadows that are common to both settings.

Here is a brief outline of the paper. Section 2 reviews the results that we need from the graded representation theory of the cyclotomic KLR algebras of type $A$. In particular, we introduce the $q$-character map for these algebras and use them to give a new proof that the graded decomposition matrices in positive characteristic factor through those in characteristic zero. Section 3 introduces the cyclotomic Hecke algebras and their character map. We define seminormal bases and use these to give a new factorisation of the Gram determinants that is compatible with taking characters. Section 4 contains the heart of the paper. We properly introduce the Jantzen filtrations and the Jantzen characters and then prove our main theorem. Finally, Sect. 5 shows how to prove the "classical" Jantzen sum formula using the framework of this paper. As the classical Jantzen sum formula is more explicit it takes more effort to prove than our positive sum formula, but it is worth the effort because the new "classical" sum formula that we obtain is nicer than the existing sum formula in the literature [19, Theorem 4.6].

The definition of cyclotomic Hecke algebras used in this paper includes the degenerate cyclotomic algebras of [4] as the special case when the Hecke algebra parameter is one. Jantzen filtrations for these algebras have not appeared in the literature previously, so even the "classical" Jantzen sum formula that we obtain in the degenerate case is completely new.

\section{Cyclotomic KLR algebras and their modules}

In this section we introduce the cyclotomic KLR algebras of type $A$, which are isomorphic to the cyclotomic Hecke algebras of type $A$. We will use these algebras to define the modules $E_{f, e}^{\mu}$ from the introduction and to prove some combinatorial identities that we use in Sect. 4.4 to compute characters for the Jantzen filtration.

\subsection{Graded modules and algebras}

In this paper all modules are finitely generated modules over a commutative ring with one. A graded module $M$ is a module with a $\mathbb{Z}$-grading $M=\bigoplus_{d \in \mathbb{Z}} M_{d}$. Similarly, graded algebra $A$ is a $\mathbb{Z}$-graded algebra $A=\bigoplus_{d \in \mathbb{Z}} A_{d}$ with $A_{d} A_{e} \subseteq A_{d+e}$, for all $d, e \in \mathbb{Z}$. If $A$ is a graded algebra then a graded $A$-module is a graded (right) $A$-module such that $M_{d} A_{e} \subseteq M_{d+e}$, for $d, e \in \mathbb{Z}$. If $F$ is a field and $M$ is a graded $F$-module then the graded dimension of $M$ is

$$
\operatorname{dim}_{q} M=\sum_{d \in \mathbb{Z}}\left(\operatorname{dim} M_{d}\right) q^{d} \in \mathbb{N}\left[q, q^{-1}\right],
$$

where $q$ is an indeterminate over $\mathbb{Z}$. Given an integer $s$ let $q^{s} M$ be the graded module obtained from $M$ by shifting the grading of $M$ by $s$ so that $\left(q^{s} M\right)_{d}=M_{d-s}$. More generally, given a Laurent polynomial $s(q)=\sum_{d} s_{d} q^{d} \in \mathbb{N}\left[q, q^{-1}\right]$ let

$$
s(q) M=\bigoplus_{d \in \mathbb{Z}}\left(q^{d} M\right)^{\oplus s_{d}} .
$$


Then $\operatorname{dim}_{q} s(q) M=s(q) \operatorname{dim}_{q} M$. Let $A$ - grMod be the category of finitely generated graded (right) $A$-modules with degree preserving homomorphisms.

If $A$ is a graded algebra let $\underline{A}$ be the (ungraded) algebra obtained by forgetting the grading on $A$. Similarly, if $M$ is a graded $A$-module let $\underline{M}$ be the (ungraded) $\underline{A}$-module obtained by forgetting the grading on $M$. This defines an exact functor from the category of finite dimensional graded $A$-modules to the category of finite dimensional $\underline{A}$-modules.

\subsection{Graded cellular algebras}

Cellular algebras, which were introduced by Graham and Lehrer [13], provide a convenient framework for constructing the simple modules of an algebra. The natural extension of their definitions and results to the graded setting was given in [14]. The proofs of all of the results in this section can be found in these two papers.

Let $R$ be a commutative ring with one.

Definition 2.1 (Graham and Lehrer [13, Definition 1.1], Hu-Mathas [14, Definition 2.1]) Let $A$ be a $\mathbb{Z}$-graded $R$-algebra that is free and of finite rank. A graded cell datum for $A$ is an ordered quadruple $(\mathcal{P}, T, C$, deg) where $(\mathcal{P}, \geq)$ is a finite poset, $T(\lambda)$ is a finite set for $\lambda \in \mathcal{P}$,

$$
C: \coprod_{\lambda \in \mathcal{P}} T(\lambda) \times T(\lambda) \longrightarrow A ;(\mathfrak{s}, \mathfrak{t}) \mapsto c_{\mathfrak{s t}} \quad \text { and } \quad \operatorname{deg}: \coprod_{\lambda \in \mathcal{P}} T(\lambda) \longrightarrow \mathbb{Z} ; \mathfrak{t} \mapsto \operatorname{deg} \mathfrak{t}
$$

are maps such that $C$ is injective and the following hold:

$\left.C_{0}\right)$ For $\mathfrak{s}, \mathfrak{t} \in T(\lambda)$ and $\lambda \in \mathcal{P}$, the element $c_{\mathfrak{s t}} \in A$ is homogeneous of degree $\operatorname{deg} \mathfrak{s}+\operatorname{deg} \mathfrak{t}$.

$C_{1}$ ) The set $\left\{c_{\mathfrak{s t}} \mid \mathfrak{s}, \mathfrak{t} \in T(\lambda), \lambda \in \mathcal{P}\right\}$ is a basis of $A$.

$C_{2}$ ) If $a \in A, \mathfrak{s}, \mathfrak{t} \in T(\lambda)$ and $\lambda \in \mathcal{P}$ then there exist scalars $r_{\mathfrak{t}}(a) \in R$, that do not depend on $\mathfrak{s}$, such that

$$
c_{\mathfrak{s t}} a \equiv \sum_{\mathfrak{v} \in T(\lambda)} r_{\mathfrak{t v}}(a) c_{\mathfrak{s} \mathfrak{v}} \quad\left(\bmod A^{>\lambda}\right)
$$

where $A^{>\lambda}$ is the $R$-submodule of $A$ spanned by $\left\{c_{\mathfrak{u} \mathfrak{v}} \mid \mathfrak{u}, \mathfrak{v} \in T(\mu)\right.$ for $\left.\mu>\lambda\right\}$.

$\left.C_{3}\right)$ There is an unique algebra anti-isomorphism $*: A \longrightarrow A$ such that $c_{\mathfrak{s t}}^{*}=c_{\mathfrak{t s}}$, for all $\mathfrak{s}, \mathfrak{t} \in T(\lambda)$ and $\lambda \in \mathcal{P}$.

A graded cellular algebra is an algebra that has a graded cell datum.

A cellular algebra is a graded cellular algebra that is concentrated in degree zero. That is, it has a graded cell datum $(\mathcal{P}, T, C$, deg) with $\operatorname{deg} \mathfrak{s}=0$. for all $\mathfrak{s}$. In this case, $(\mathcal{P}, T, C)$ is a cell datum for $A$.

In particular, note that if $A$ is a graded cellular algebra then, forgetting the grading, $\underline{A}$ is a cellular algebra.

Let $A$ be a (graded) cellular algebra with cell datum $(\mathcal{P}, T, C$, deg). Using the definitions it is easy to see that for each $\lambda \in \mathcal{P}$ there exist a (right) cell module $C^{\lambda}$ with basis $\left\{c_{\mathfrak{t}} \mid \mathfrak{t} \in T(\lambda)\right\}$ and with $A$-action

$$
c_{\mathfrak{t}} a=\sum_{\mathfrak{v} \in T(\lambda)} r_{\mathfrak{t v}}(a) c_{\mathfrak{v}}, \quad \text { for } a \in A
$$


where the scalars $r_{\mathfrak{t v}}(a) \in R$ are from $\left(\mathrm{C}_{2}\right)$. Similarly, let $C^{\lambda *}$ be the left cell module indexed by $\lambda$, which is isomorphic to $C^{\lambda}$ as a vector space and where the $A$-action is given by

$$
a c_{\mathfrak{t}}=\sum_{\mathfrak{v} \in T(\lambda)} r_{\mathfrak{t v}}\left(a^{*}\right) c_{\mathfrak{v}}, \quad \text { for } a \in A,
$$

Extending the notation from $\left(\mathrm{C}_{2}\right)$, let $A^{\geq \lambda}=\left\langle c_{\mathfrak{u} \mathfrak{v}}\right| \mathfrak{u}, \mathfrak{v} \in T(\mu)$ for $\left.\mu \geq \lambda\right\rangle_{R}$ and define the $(A, A)$-bimodule map $c_{\lambda}$ by

$$
c_{\lambda}: C^{\lambda *} \otimes C^{\lambda} \longrightarrow A^{\geq \lambda} / A^{>\lambda} ; c_{\mathfrak{s}} \otimes c_{\mathfrak{t}} \mapsto c_{\mathfrak{s t}}+A^{>\lambda}, \quad \text { for } \mathfrak{s}, \mathfrak{t} \in T(\lambda) .
$$

Using $\left(\mathrm{C}_{2}\right)$ and $\left(\mathrm{C}_{3}\right)$, it follows that $C^{\lambda}$ has a homogeneous symmetric bilinear form $\langle\rangle:, C^{\lambda} \times$ $C^{\lambda} \longrightarrow R$ of degree zero such that

$$
x \cdot c_{\lambda}(y \otimes z)=\langle x, y\rangle z, \quad \text { for } x, y, z \in C^{\lambda} .
$$

In particular, $c_{\mathfrak{t}} c_{\mathfrak{u} \mathfrak{v}}=\left\langle c_{\mathfrak{t}}, c_{\mathfrak{u}}\right\rangle c_{\mathfrak{v}}$, for $\mathfrak{t}, \mathfrak{u}, \mathfrak{v} \in T(\lambda)$. This form is associative in the sense that $\langle x a, y\rangle=\left\langle x, y a^{*}\right\rangle$, for all $x, y \in C^{\lambda}$ and $a \in A$. Hence,

$$
\operatorname{rad} C^{\lambda}=\left\{x \in C^{\lambda} \mid\langle x, y\rangle=0 \text { for all } y \in C^{\lambda}\right\}
$$

is a (graded) $A$-submodule of $C^{\lambda}$. Set $D^{\lambda}=C^{\lambda} / \operatorname{rad} C^{\lambda}$. Then $D^{\lambda}$ is a (graded) $A$-module.

Let $D$ be an irreducible graded $A$-module. Then $q^{k} D$ is a non-isomorphic irreducible graded $A$-module, for $k \in \mathbb{Z} \backslash\{0\}$. If $M$ is a graded $A$-module then the graded decomposition multiplicity of $D$ in $M$ is the Laurent polynomial

$$
[M: D]_{q}=\sum_{k \in \mathbb{Z}}\left[M: q^{k} D\right] q^{k} \in \mathbb{N}\left[q, q^{-1}\right]
$$

where $\left[M: q^{k} D\right]$ is the multiplicity of $q^{k} D$ as a composition factor of $M$.

The main results from the theory of (graded) cellular algebras are:

Theorem 2.4 (Graham and Lehrer [13, Theorem 3.4] and [14, Theorem 2.10]) Let $F$ be a field and suppose that $A$ is a graded cellular algebra. Then:

(a) Let $\mu \in \mathcal{P}$ and suppose that $D^{\mu} \neq 0$. Then $D^{\mu}$ is a self-dual absolutely irreducible graded A-module.

(b) Let $\mathcal{P}_{0}=\left\{\mu \in \mathcal{P} \mid D^{\mu} \neq 0\right\}$. Then $\left\{q^{k} D^{\mu} \mid \mu \in \mathcal{P}_{0}\right.$ and $\left.k \in \mathbb{Z}\right\}$ is a complete set of pairwise non-isomorphic irreducible graded A-modules.

(c) If $\lambda \in \mathcal{P}$ and $\mu \in \mathcal{P}_{0}$. Then $\left[S^{\mu}: D^{\mu}\right]_{q}=1$ and $\left[S^{\lambda}: D^{\mu}\right]_{q} \neq 0$ only if $\lambda \geq \mu$.

We need the theory of graded cellular algebras only to explain the definition of the Specht modules of the cyclotomic Hecke algebras. The bilinear form on the Specht modules is the key to defining their Jantzen filtrations. Our main results also use the graded Specht modules, although we have to approach this indirectly because the bilinear form on the graded Specht modules is degenerate unless the graded Specht module is irreducible.

\subsection{Cyclotomic KLR algebras}

Fix an integer $e \in\{2,3,4, \ldots\} \cup\{\infty\}$ and let $\Gamma=\Gamma_{e}$ be the quiver with vertex set $I=\mathbb{Z} / e \mathbb{Z}$ (we set $e \mathbb{Z}=\{0\}$ when $e=\infty$ ), and with edges $i \rightarrow i+1$, for $i \in I$. To the quiver $\Gamma$ we attach the standard Lie theoretic data of a Cartan matrix $\left(c_{i j}\right)_{i, j \in I}$, fundamental weights $\left\{\Lambda_{i} \mid i \in I\right\}$, 
the positive weight lattice $P_{e}^{+}=\sum_{i \in I} \mathbb{N} \Lambda_{i}$, the positive root lattice $Q_{e}^{+}=\bigoplus_{i \in I} \mathbb{N} \alpha_{i}$ and we let $(\cdot, \cdot)$ be normalised invariant form determined by

$$
\left(\alpha_{i}, \alpha_{j}\right)=c_{i j} \quad \text { and } \quad\left(\Lambda_{i}, \alpha_{j}\right)=\delta_{i j}, \quad \text { for } i, j \in I .
$$

Let $Q_{e, n}^{+}=\left\{\beta \in Q_{e}^{+} \mid \sum_{i \in I}\left(\Lambda_{i}, \beta\right)=n\right\}$ and for $\beta \in Q_{e, n}^{+}$let $I^{\beta}=\left\{\mathbf{i} \in I^{n} \mid \beta=\right.$ $\left.\alpha_{i_{1}}+\cdots+\alpha_{i_{n}}\right\}$.

Let $\mathfrak{S}_{n}$ be the symmetric group of degree $n$, which we consider as a Coxeter group with its standard set of Coxeter generators $\left\{s_{1}, \ldots, s_{n-1}\right\}$, where $s_{r}=(r, r+1)$ for $1 \leq r<n$. Let $\mathfrak{S}_{n}$ act on $I^{n}$ by place permutations so that if $\mathbf{i}=\left(i_{1}, \ldots, i_{n}\right) \in I^{n}$ then $w \cdot \mathbf{i}=$ $\left(i_{w(1)}, \ldots, i_{w(n)}\right) \in I^{n}$.

Following Rouquier [38, Definition 3.2.2], let $u$ and $v$ be indeterminates and for $i, j \in I$ define polynomials

$$
Q_{i j}(u, v)= \begin{cases}(u-v)(v-u) & \text { if } i \leftrightarrows j, \\ u-v & \text { if } i \rightarrow j, \\ v-u & \text { if } i \leftarrow j, \\ 0 & \text { if } i=j, \\ 1 & \text { otherwise }\end{cases}
$$

To define the cyclotomic KLR algebras of type $A$, fix a non-negative integer $n \geq 0$ and a dominant weight $\Lambda \in P_{e}^{+}$and let $\ell=\sum_{i \in I}\left(\Lambda, \alpha_{i}\right)$.

Definition 2.5 (Khovanov and Lauda [26, §2] and Rouquier [38, Definition 3.2.2]) Let $e \in$ $\{2,3, \ldots\} \cup\{\infty\}$ and fix $\beta \in Q_{e, n}^{+}$. The cyclotomic KLR algebra $\mathscr{R}_{\beta}^{\Lambda}$ of type $\Gamma$, determined by the weights $(\Lambda, \beta)$, is the unital associative $\mathbb{Z}$-algebra with generators $\left\{\psi_{1}, \ldots, \psi_{n-1}\right\} \cup$ $\left\{y_{1}, \ldots, y_{n}\right\} \cup\left\{e(\mathbf{i}) \mid \mathbf{i} \in I^{\beta}\right\}$ and relations

$$
\begin{aligned}
& y_{1}^{\left(\Lambda, \alpha_{i_{1}}\right)} e(\mathbf{i})=0 \quad e(\mathbf{i}) e(\mathbf{j})=\delta_{\mathbf{i j}} e(\mathbf{i}), \quad \sum_{\mathbf{i} \in I^{\beta}} e(\mathbf{i})=1, \\
& y_{r} e(\mathbf{i})=e(\mathbf{i}) y_{r}, \quad \psi_{r} e(\mathbf{i})=e\left(s_{r} \cdot \mathbf{i}\right) \psi_{r}, \quad y_{r} y_{s}=y_{s} y_{r},
\end{aligned}
$$

$$
\begin{aligned}
& \psi_{r} \psi_{s}=\psi_{s} \psi_{r} \text { if }|r-s|>1, \quad \psi_{r} y_{s}=y_{s} \psi_{r} \text { if } s \neq r, r+1, \\
& \quad \psi_{r} y_{r+1} e(\mathbf{i})=\left(y_{r} \psi_{r}+\delta_{i_{r} i_{r+1}}\right) e(\mathbf{i}), \quad y_{r+1} \psi_{r} e(\mathbf{i})=\left(\psi_{r} y_{r}+\delta_{i_{r} i_{r+1}}\right) e(\mathbf{i}), \\
& \psi_{r}^{2} e(\mathbf{i})=Q_{i_{r} i_{r+1}}\left(y_{r}, y_{r+1}\right) e(\mathbf{i}) \\
& \left(\psi_{r} \psi_{r+1} \psi_{r}-\psi_{r+1} \psi_{r} \psi_{r+1}\right) e(\mathbf{i}) \\
& =\delta_{i_{r} i_{r+1}} \frac{Q_{i_{r}, i_{r+1}}\left(y_{r+2}, y_{r+1}\right)-Q_{i_{r}, i_{r+1}}\left(y_{r}, y_{r+1}\right)}{y_{r}-y_{r+2}} e(\mathbf{i})
\end{aligned}
$$

for $\mathbf{i}, \mathbf{j} \in I^{\beta}$ and all admissible $r$ and $s$. Set $\mathscr{R}_{n}^{\Lambda}=\bigoplus_{\beta \in Q_{e, n}^{+}} \mathscr{R}_{\beta}^{\Lambda}$.

The reader can check that the right hand side of the last relation is a polynomial in $y_{r}, y_{r+1}$ and $y_{r+2}$. Importantly, the algebras $\mathscr{R}_{\beta}^{\Lambda}$ and $\mathscr{R}_{n}^{\Lambda}$ are $\mathbb{Z}$-graded, where the grading is determined by

$$
\operatorname{deg} e(\mathbf{i})=0, \quad \operatorname{deg} y_{r}=2 \text { and } \operatorname{deg} \psi_{r} e(\mathbf{i})=-\left(\alpha_{i_{r}}, \alpha_{i_{r+1}}\right),
$$

for all admissible $\mathbf{i} \in I^{n}$ and $1 \leq r \leq n$. If $R$ is any ring let $\mathscr{R}_{\beta}^{\Lambda}(R)=\mathscr{R}_{\beta}^{\Lambda} \otimes_{\mathbb{Z}} R$ and $R_{n}^{\Lambda}(R)=R_{n}^{\Lambda} \otimes_{\mathbb{Z}} R$ be the corresponding cyclotomic KLR algebras over $R$.

From the relations, $\mathscr{R}_{n}^{\Lambda}$ has a unique homogeneous anti-automorphism $*$ of degree 0 that fixes all of the generators of $\mathscr{R}_{n}^{\Lambda}$.

For each $w \in \mathfrak{S}_{n}$ fix a reduced expression $w=s_{r_{1}} \ldots s_{r_{k}}$ for $w$ (that is, fix such a word with $k$ minimal), and define $\psi_{w}=\psi_{r_{1}} \ldots \psi_{r_{k}} \in \mathscr{R}_{n}^{\Lambda}$. In general, the element $\psi_{w}$ depends 
on the choice of reduced expression but any fixed choice of reduced expression suffices for the results that follow.

\subsection{Formal characters}

Formal characters are a useful tool in the representation theory of the affine Hecke algebra. We need an analogue of these characters for $\mathscr{R}_{n}^{\Lambda}$-modules. To this end, let $\mathcal{A}=\mathbb{Z}\left[q, q^{-1}\right]$ and let $\mathcal{A}\left[I^{n}\right]=\mathcal{A}\left[\mathbf{i} \mid \mathbf{i} \in I^{n}\right]$ be the free $\mathcal{A}$-module with basis $\left\{\mathbf{i} \mid \mathbf{i} \in I^{n}\right\}$.

If $F$ is a field let $\operatorname{Rep} \mathscr{R}_{n}^{\Lambda}(F)$ be the Grothendieck group of finitely generated graded $\mathscr{R}_{n}^{\Lambda}(F)$-modules and if $M$ is an $\mathscr{R}_{n}^{\Lambda}$-module let $[M]$ be its image in $\operatorname{Rep} \mathscr{R}_{n}^{\Lambda}(F)$. Then $\operatorname{Rep} \mathscr{R}_{n}^{\Lambda}(F)$ is the free $\mathcal{A}$-module with basis $\left\{\left[\mathbb{D}^{\mu}\right] \mid \mu \in \mathcal{K}_{n}^{\Lambda}\right\}$, where $q$ acts on $\operatorname{Rep} \mathscr{R}_{n}^{\Lambda}(F)$ by grading shift, so that $[q M]=q[M]$, for $M \in \operatorname{Rep} \mathscr{R}_{n}^{\Lambda}(F)$. The simple modules $\left\{\left[\mathbb{D}^{\mu}\right] \mid \mu \in\right.$ $\mathcal{K}_{n}^{\Lambda}$ \} are introduced in Definition 2.14.

Let $\mathscr{L}_{n}$ be the positively graded commutative subalgebra of $\mathscr{R}_{n}^{\Lambda}$ that is generated by $y_{1}, \ldots, y_{n}$ and the idempotents $e(\mathbf{i})$, for $\mathbf{i} \in I^{n}$. It is easy to see that the irreducible representations of $\mathscr{L}_{n}$ are indexed by the $n$-tuples $\mathbf{i} \in I^{n}$ such that $e(\mathbf{i}) \neq 0$. Let $\operatorname{Rep} \mathscr{L}_{n}$ be the Grothendieck group of finitely generated graded $\mathscr{L}_{n}$-modules where $q$ acts by grading shift, which we view as a free $\mathcal{A}$-submodule of $\mathcal{A}\left[I^{n}\right]$.

Definition 2.6 The graded (formal) character is the linear map $\operatorname{ch}_{q}: \operatorname{Rep} \mathscr{R}_{n}^{\Lambda} \longrightarrow \operatorname{Rep} \mathscr{L}_{n}$ given by

$$
\operatorname{ch}_{q} M=\sum_{\mathbf{i} \in I^{n}}\left(\operatorname{dim}_{q} M e(\mathbf{i})\right) \mathbf{i}, \quad \text { for } M \in \operatorname{Rep} \mathscr{R}_{n}^{\Lambda}
$$

The $\operatorname{map} \mathrm{ch}_{q}$ can be viewed as an exact functor, namely the restriction functor, from the category of (graded) $\mathscr{R}_{n}^{\Lambda}$-modules to the category of graded $\mathscr{L}_{n}$-modules. For our purposes it is enough to think of $\operatorname{ch}_{q}$ as a linear map $\operatorname{ch}_{q}: \operatorname{Rep} \mathscr{R}_{n}^{\Lambda} \longrightarrow \mathcal{A}\left[I^{n}\right]$.

Proposition 2.7 Let $F$ be a field. Then the graded character map $\operatorname{ch}_{q}: \operatorname{Rep} \mathscr{R}_{n}^{\Lambda}(F) \longrightarrow \mathcal{A}\left[I^{n}\right]$ is injective.

Proof Khovanov and Lauda [26, Theorem 3.17] state this result for the Grothendieck group of the corresponding affine KLR algebra, however, this immediately implies the result for the quotient cyclotomic KLR Hecke algebra $\mathscr{R}_{n}^{\Lambda}(F)$. A more complete proof, in a different but closely related context, can be found in [27, Theorem 3.3.1].

\subsection{Tableau combinatorics}

Before we can define a homogeneous basis for $\mathscr{R}_{n}^{\Lambda}$, and introduce the graded Specht modules for $\mathscr{R}_{n}^{\Lambda}$, we need to introduce the combinatorics of standard tableaux. This combinatorics plays a key role in proofs of our main results. Recall that we fixed integers $n$ and $\ell$ in the paragraph before Definition 2.5.

A partition of $m$ is a weakly decreasing sequence $\lambda=\left(\lambda_{1}, \lambda_{2}, \ldots\right)$ of non-negative integers such that $|\lambda|=\lambda_{1}+\lambda_{2}+\cdots=m$. An $\ell$-partition of $n$ is an $\ell$-tuple $\lambda=\left(\lambda^{(1)}, \ldots, \lambda^{(\ell)}\right)$ of partitions such that $\left|\lambda^{(1)}\right|+\cdots+\left|\lambda^{(\ell)}\right|=n$. We identify the $\ell$-partition $\lambda$ with its diagram, which is the set of nodes $\left\{(l, r, c) \mid 1 \leq c \leq \lambda_{r}^{(l)}\right.$ for $\left.1 \leq l \leq \ell\right\}$. We think of the diagram of $\lambda$ as an $\ell$-tuple of left-justified arrays of boxes. As shown in (2.8), we use the "English convention" for tableaux. This allows us to talk of the components, rows and columns of $\lambda$. 
The set of $\ell$-partitions of $n$ is a poset under the dominance order $\unrhd$, where $\lambda \unrhd \boldsymbol{\mu}$ if

$$
\sum_{k=1}^{l-1}\left|\lambda^{(k)}\right|+\sum_{j=1}^{i} \lambda_{j}^{(l)} \geq \sum_{k=1}^{l-1}\left|\mu^{(k)}\right|+\sum_{j=1}^{i} \mu_{j}^{(l)},
$$

for $1 \leq l \leq \ell$ and $i \geq 1$. If $\lambda \unrhd \mu$ and $\lambda \neq \mu$ then write $\lambda \triangleright \mu$. Let $\mathcal{P}_{n}^{\Lambda}=\mathcal{P}_{\ell, n}^{\Lambda}$ be the set of $\ell$-partitions of $n$, which we consider as a poset under dominance.

Fix $\lambda \in \mathcal{P}_{n}^{\Lambda}$. A $\lambda$-tableau is a bijective map $\mathfrak{t}: \lambda \longrightarrow\{1,2, \ldots, n\}$, which we identify with a labelling of (the diagram of) $\lambda$ by $\{1,2, \ldots, n\}$. Let Shape $(\mathfrak{t})=\lambda$ be the shape of $t$. For example,

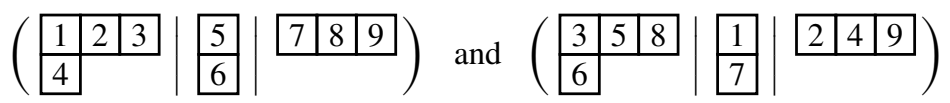

are both $\lambda$-tableaux, where $\lambda=\left(3,1\left|1^{2}\right| 3\right) \in \mathcal{P}_{13}^{\Lambda}$ and $\ell=3$. A $\lambda$-tableau is standard if, in each component, its entries increase along rows and down columns. For example, both of the tableaux above are standard. Let $\operatorname{Std}(\lambda)$ be the set of standard $\lambda$-tableaux. If $\mathcal{P}$ is any set of $\ell$-partitions let $\operatorname{Std}(\mathcal{P})=\bigcup_{\lambda \in \mathcal{P}} \operatorname{Std}(\lambda)$. Similarly $\operatorname{set} \operatorname{Std}^{2}(\mathcal{P})=\{(\mathfrak{s}, \mathfrak{t}) \mid \mathfrak{s}, \mathfrak{t} \in$ $\operatorname{Std}(\lambda)$ for $\lambda \in \mathcal{P}\}$.

If $\mathfrak{t}$ is a $\lambda$-tableau set Shape $(\mathfrak{t})=\lambda$ and let $\mathfrak{t}_{\downarrow m}$ be the subtableau of $\mathfrak{t}$ that contains the numbers $\{1,2, \ldots, m\}$. If $\mathfrak{t}$ is a standard $\lambda$-tableau then Shape $\left(\mathfrak{t}_{\downarrow m}\right)$ is an $\ell$-partition for all $m \geq 0$. Extend the dominance ordering to $\operatorname{Std}\left(\mathcal{P}_{n}^{\Lambda}\right)$ by defining

$$
\mathfrak{s} \unrhd \mathfrak{t} \quad \text { if Shape }\left(\mathfrak{s}_{\downarrow}\right) \unrhd \operatorname{Shape}\left(\mathfrak{t}_{\downarrow}\right) \text { for } 1 \leq m \leq n .
$$

As before, write $\mathfrak{s} \triangleright \mathfrak{t}$ if $\mathfrak{s} \unrhd \mathfrak{t}$ and $\mathfrak{s} \neq \mathfrak{t}$.

Even though we write $\mathcal{P}_{n}^{\Lambda}$ for the set of $\ell$-partitions of $n$, so far none of the definitions in this section depend on $\Lambda \in P_{e}^{+}$. A multicharge for $\Lambda$ is an $\ell$-tuple $\kappa=\left(\kappa_{1}, \ldots, \kappa_{\ell}\right) \in \mathbb{Z}^{\ell}$ such that

$$
\left(\Lambda, \alpha_{i}\right)=\#\left\{1 \leq l \leq \ell \mid \kappa_{l} \equiv i(\bmod e)\right\}, \quad \text { for all } i \in I .
$$

The residue of a node $A=(l, r, c) \in \lambda$ is $\mathrm{r}(A)=\kappa_{l}+c-r+e \mathbb{Z} \in I$. If $\mathfrak{t}$ is a standard $\lambda$-tableau and $\mathfrak{t}(A)=m$, where $1 \leq m \leq n$, then the residue of $m$ in $\mathfrak{t}$ is $\mathrm{r}_{m}(\mathfrak{t})=\mathrm{r}(A)$. The residue sequence of $\mathfrak{t}$ is $r(\mathfrak{t})=\left(r_{1}(\mathfrak{t}), r_{2}(\mathfrak{t}), \ldots, r_{n}(\mathfrak{t})\right) \in I^{n}$. Set

$$
\left.\operatorname{Std}_{\mathbf{i}}(\lambda)=\{\mathfrak{t} \in \operatorname{Std}(\lambda) \mid r(\mathfrak{t})=\mathbf{i}\}\right) \text { and } \operatorname{Std}_{\mathbf{i}}\left(\mathcal{P}_{n}^{\Lambda}\right)=\bigcup_{\lambda \in \mathcal{P}_{n}^{\Lambda}} \operatorname{Std}_{\mathbf{i}}(\lambda) .
$$

A node $A$ is an addable $i$-node of $\lambda$ if $\mathrm{r}(A)=i, A \notin \lambda$ and $\lambda \cup\{A\}$ is the (diagram of) a $\ell$-partition of $n+1$. Similarly, a node $B$ is a removable $i$-node of $\lambda$ if $\operatorname{r}(B)=i$, $B \in \lambda$ and $\lambda \backslash\{B\}$ is a $\ell$-partition of $n-1$. Let $\operatorname{Add}_{i}(\lambda)$ and $\operatorname{Rem}_{i}(\lambda)$ be the sets of addable and removable $i$-nodes of $\lambda$. Let $\leq$ be the lexicographic order on the set of nodes. If $A$ is a removable node of $\lambda$ define

$$
d_{A, e}(\lambda)=\#\left\{B \in \operatorname{Add}_{i}(\lambda) \mid A<B\right\}-\#\left\{B \in \operatorname{Rem}_{i}(\lambda) \mid A<B\right\} .
$$

Following Brundan, Kleshchev and Wang [6, §3.5], define the $e$-degree of a standard $\lambda$ tableau $\mathfrak{t}$ by

$$
\operatorname{deg}_{e}(\mathfrak{t})= \begin{cases}\operatorname{deg}_{e}\left(\mathfrak{t}_{\downarrow}(n-1)\right)+d_{A, e}(\boldsymbol{\mu}), & \text { if } n>0, \\ 0, & \text { if } n=0\end{cases}
$$


where $A=\mathfrak{t}^{-1}(n)$ is the node in $\mathfrak{t}$ that contains $n$, For convenience, set $\operatorname{deg}_{0}(\mathfrak{t})=0$ for all $\mathfrak{t}$.

There is unique standard $\lambda$-tableaux $\mathfrak{t}^{\lambda}$ such that $\mathfrak{t}^{\lambda} \unrhd \mathfrak{t}$, for all $\mathfrak{t} \in \operatorname{Std}(\lambda)$. The tableau $\mathfrak{t}^{\lambda}$ has the numbers $1,2, \ldots, n$ entered in order from left to right along the rows of $\mathfrak{t}^{\lambda^{(1)}}$, and then $\mathfrak{t}^{\lambda^{(2)}}, \ldots, \mathfrak{t}^{\lambda^{(\ell)}}$. For example, the first tableau in 2.5 is $\mathfrak{t}^{\lambda}$ for $\lambda=\left(3,1\left|1^{2}\right| 3\right)$. Given a standard $\lambda$-tableau $\mathfrak{t}$ define a permutation $d(\mathfrak{t}) \in \mathfrak{S}_{n}$ by $\mathfrak{t}=\mathfrak{t}^{\lambda} d(\mathfrak{t})$.

\subsection{Specht modules, simple modules and almost simple modules}

The main results in this paper give explicit, cancellation free, descriptions of the Jantzen sum formula for the (ungraded) Specht modules. This section defines the modules that we use to describe these filtrations.

As we now recall, the algebra $\mathscr{R}_{n}^{\Lambda}$ is a graded cellular algebra in the sense of Sect. 2.2.

For $\lambda \in \mathcal{P}_{n}^{\Lambda}$ set $\mathbf{i}^{\lambda}=\mathrm{r}\left(\mathfrak{t}^{\lambda}\right)=\left(i_{1}^{\lambda}, \ldots, i_{n}^{\lambda}\right)$. For $1 \leq m \leq n$ set $\lambda_{\downarrow m}=\operatorname{Shape}\left(\mathfrak{t}_{\downarrow m}^{\lambda}\right)$ and let $\alpha_{m}^{\lambda} \in \lambda$ be the node such that $\mathfrak{t}^{\lambda}\left(\alpha_{m}^{\lambda}\right)=m$. Define the polynomial

$$
y^{\lambda}=\prod_{k=1}^{n} y_{r}^{a_{k}(\lambda)}, \quad \text { where } a_{k}(\lambda)=\#\left\{\alpha \in \operatorname{Add}_{i_{k}^{\lambda}}\left(\lambda_{\downarrow k}\right) \mid \alpha<\alpha_{k}^{\lambda}\right\} .
$$

Then $y^{\lambda}$ is homogeneous of degree $2 \operatorname{deg} \mathfrak{t}^{\lambda}$. For $(\mathfrak{s}, \mathfrak{t}) \in \operatorname{Std}^{2}(\lambda)$ define

$$
\psi_{\mathfrak{s t}}=\psi_{d(\mathfrak{s})}^{*} e\left(\mathbf{i}^{\lambda}\right) y^{\lambda} \psi_{d(\mathfrak{t})},
$$

By construction, $\psi_{\mathfrak{s t}}$ is homogeneous and one can show that $\operatorname{deg}_{e} \psi_{s \mathfrak{t}}=\operatorname{deg}_{e} \mathfrak{s}+\operatorname{deg} \mathrm{t}$. Hu and the author [14] proved that these elements give a graded cellular basis for $\mathscr{R}_{n}^{\Lambda}(F)$, when $F$ is a field. Building on this result, $\mathrm{Li}[30]$ proved that $\mathscr{R}_{n}^{\Lambda}$ is a $\mathbb{Z}$-free graded cellular algebra.

Theorem 2.11 (Li [30, Theorem 1.1]) The algebra $\mathscr{R}_{n}^{\Lambda}$ is free as an $\mathbb{Z}$-module of rank $\ell^{n} n$ !. Moreover, $\mathscr{R}_{n}^{\Lambda}$ is a graded cellular algebra with homogeneous cellular basis $\left\{\psi_{\mathfrak{s t}} \mid(\mathfrak{s}, \mathfrak{t}) \in\right.$ $\left.\operatorname{Std}^{2}\left(\mathcal{P}_{n}^{\Lambda}\right)\right\}$

Subsequently, Kang and Kashiwara [23, Theorem 4.5] generalised this result and proved that the cyclotomic KLR Hecke algebras indexed by symmetrisable Cartan matrices are $\mathbb{Z}$-free. For this paper, the fact that $\mathscr{R}_{n}^{\Lambda}(\mathbb{Z})$ is a cellular algebra is very important.

Since $\mathscr{R}_{n}^{\Lambda}$ is a cellular algebra, if $a \in \mathscr{R}_{n}^{\Lambda}$ and $(\mathfrak{s}, \mathfrak{t}) \in \operatorname{Std}^{2}(\lambda)$, for $\lambda \in \mathcal{P}_{n}^{\Lambda}$, then we can write

$$
\psi_{\mathfrak{s t}} a \equiv \sum_{\mathfrak{u} \in \operatorname{Std}(\lambda)} r_{\mathfrak{u}} \psi_{\mathfrak{u t}} \quad\left(\bmod \mathscr{R}_{n}^{\triangleright \lambda}\right)
$$

where the scalar $r_{\mathfrak{u}} \in \mathbb{Z}$ depends only on $a, \mathfrak{s}$ and $\mathfrak{u}$ (and not $\mathfrak{t}$ ) and where $\mathscr{R}_{n}^{\triangleright \lambda}$ is the two-sided ideal of $\mathscr{R}_{n}^{\Lambda}$ spanned by $\left\{\psi_{\mathfrak{u v}} \mid(\mathfrak{u}, \mathfrak{v}) \in \operatorname{Std}(\boldsymbol{\mu})\right.$ where $\left.\boldsymbol{\mu} \triangleright \lambda\right\}$. Define the graded Specht module $\mathbb{S}_{\mathbb{Z}}^{\lambda}$ to the free $\mathbb{Z}$-module with homogeneous basis $\left\{\psi_{\mathfrak{s}} \mid \mathfrak{s} \in \operatorname{Std}(\lambda)\right\}$, where $\operatorname{deg} \psi_{\mathfrak{s}}=\operatorname{deg}_{e} \mathfrak{s}$, and with $\mathscr{R}_{n}^{\Lambda}$-action

$$
\psi_{\mathfrak{s}} a=\sum_{\mathfrak{u} \in \operatorname{Std}(\lambda)} r_{\mathfrak{u}} \psi_{\mathfrak{u}}, \quad \text { for } a \in \mathscr{R}_{n}^{\Lambda} \text { and } \mathfrak{s} \in \operatorname{Std}(\lambda) .
$$

In particular, it follows that if $\mathbf{i} \in I^{n}$ then $\psi_{\mathfrak{s}} e(\mathbf{i})=\delta_{\mathbf{i i}^{\mathfrak{s}}} \psi_{\mathfrak{s}}$.

For any ring $F$ let $\mathbb{S}_{F}^{\lambda}=\mathbb{S}_{\mathbb{Z}}^{\lambda} \otimes_{\mathbb{Z}} F$. Then $\mathbb{S}_{F}^{\lambda}$ is a graded $\mathscr{R}_{n}^{\Lambda}(F)$-module. We abuse notation and write $\psi_{\mathfrak{s}}$, instead of $\psi_{s} \otimes_{\mathbb{Z}} 1_{F}$, for the basis elements of $\mathbb{S}_{F}^{\lambda}$. 
The cellular algebra axioms imply that $\mathbb{S}_{F}^{\lambda}$ has a bilinear form $\langle\rangle:, \mathbb{S}_{F}^{\lambda} \times \mathbb{S}_{F}^{\lambda} \longrightarrow F$ determined by

$$
\left\langle\psi_{\mathfrak{s}}, \psi_{\mathfrak{t}}\right\rangle \psi_{\mathfrak{u}}=\psi_{\mathfrak{s t}} \psi_{\mathfrak{u}}, \quad \text { for } \mathfrak{s}, \mathfrak{t}, \mathfrak{u} \in \operatorname{Std}(\lambda) .
$$

This form is associative in the sense that $\langle a x, y\rangle=\left\langle x, a^{*} y\right\rangle$, for all $a \in \mathscr{R}_{n}^{\Lambda}$ and $x, y \in \mathbb{S}_{F}^{\lambda}$. Hence,

$$
\operatorname{rad} \mathbb{S}_{F}^{\lambda}=\left\{x \in \mathbb{S}_{F}^{\lambda} \mid\langle x, y\rangle=0 \text { for all } y \in \mathbb{S}_{F}^{\lambda}\right\}
$$

is a graded $\mathscr{R}_{n}^{\Lambda}(F)$-submodule of $\mathbb{S}_{F}^{\lambda}$. Observe that these definitions make sense for any ring and, in particular, these definitions are valid even over $F=\mathbb{Z}$.

Definition 2.14 Let $\mu \in \mathcal{P}_{n}^{\Lambda}$. Define $\mathbb{E}_{\mathbb{Z}}^{\mu}=\mathbb{S}_{\mathbb{Z}}^{\mu} / \operatorname{rad} \mathbb{S}_{\mathbb{Z}}^{\mu}$. For any ring $F$ define

$$
\mathbb{E}_{F}^{\mu}=\mathbb{E}_{\mathbb{Z}}^{\mu} \otimes_{\mathbb{Z}} F \quad \text { and } \quad \mathbb{D}_{F}^{\mu}=\mathbb{S}_{F}^{\lambda} / \operatorname{rad} \mathbb{S}_{F}^{\lambda} .
$$

By definition, $\mathbb{E}_{\mathbb{Z}}^{\mu}=\mathbb{D}_{\mathbb{Z}}^{\mu}$ but in positive characteristic the $\mathscr{R}_{n}^{\Lambda}(F)$-modules $\mathbb{E}_{F}^{\mu}$ and $\mathbb{D}_{F}^{\mu}$ are not necessarily isomorphic. By the theory of (graded) cellular algebras [14, Corollary 2.11], if $F$ is a field then the module $\mathbb{D}_{F}^{\mu}$ is either zero or absolutely irreducible and self-dual. Moreover, up to grading shift, every irreducible $\mathscr{R}_{n}^{\Lambda}(F)$-module arises uniquely in this way.

Let $F$ be a field and set $\mathcal{K}_{n}^{\Lambda}=\mathcal{K}_{e, n}^{\Lambda}=\left\{\boldsymbol{\mu} \in \mathcal{P}_{n}^{\Lambda} \mid \mathbb{D}_{F}^{\mu} \neq 0\right\}$. Then $\left\{q^{d} \mathbb{D}_{F}^{\mu} \mid \boldsymbol{\mu} \in \mathcal{K}_{n}^{\Lambda}\right.$ and $d \in$ $\mathbb{Z}\}$ is a complete set of pairwise non-isomorphic irreducible graded $\mathscr{R}_{n}^{\Lambda}$-modules by [14, Corollary 5.11]. (The graded irreducible $\mathscr{R}_{n}^{\Lambda}(F)$-modules were first classified in [5, Theorem 5.10].) Moreover, the set $\mathcal{K}_{n}^{\Lambda}$ is independent of the field $F$. For $\lambda \in \mathcal{P}_{n}^{\Lambda}$ and $\mu \in \mathcal{K}_{n}^{\Lambda}$ define the graded decomposition number

$$
d_{\lambda \mu}^{F, e}(q)=\left[\mathbb{S}_{F}^{\lambda}: \mathbb{D}_{F}^{\mu}\right]_{q}=\sum_{d \in \mathbb{Z}}\left[\mathbb{S}_{F}^{\lambda}: q^{d} \mathbb{D}_{F}^{\mu}\right] q^{d} \in \mathbb{N}\left[q, q^{-1}\right] .
$$

In particular, $d_{\lambda \mu}^{\mathbb{C}, e}(q)$ is a graded decomposition number for $\mathscr{R}_{n}^{\Lambda}(\mathbb{C})$ in characteristic zero. The graded decomposition matrix of $\mathscr{R}_{n}^{\Lambda}(F)$ is the unitriangular matrix $\mathrm{D}_{F, e}(q)=\left(d_{\lambda \mu}^{F, e}(q)\right)$, where $\lambda \in \mathcal{P}_{n}^{\Lambda}$ and $\boldsymbol{\mu} \in \mathcal{K}_{n}^{\Lambda}$ and where the rows are columns are ordered lexicographically.

The following result plays a key role in this paper. We give the proof, because we need the underlying ideas below, the proof is quite short, and the original reference [36] not being readily available. Given integers $d$ and $d^{\prime}$ write $d \mid d^{\prime}$ if $d^{\prime}=a d$, for some $a \in \mathbb{Z}$. In particular, note that $d \mid 0$, for all $d \in \mathbb{Z}$.

Theorem 2.16 [36, Theorem 3.7.4 and Theorem 3.7.5] Suppose that $F$ is a field and $\boldsymbol{\mu} \in \mathcal{K}_{n}^{\Lambda}$. Then:

(a) The module $\mathbb{E}_{\mathbb{Z}}^{\mu}$ is a $\mathbb{Z}$-free $\mathbb{Z}$-graded $\mathscr{R}_{n}^{\Lambda}(\mathbb{Z})$-module

(b) If $F=\mathbb{Q}$ then $\mathbb{E}_{\mathbb{Q}}^{\mu} \cong \mathbb{D}_{\mathbb{Q}}^{\mu}$ is an absolutely irreducible self-dual graded $\mathscr{R}_{n}^{\Lambda}(\mathbb{Q})$-module

(c) If $\lambda \in \mathcal{P}_{n}^{\Lambda}$ then $\left[\mathbb{S}_{F}^{\lambda}: \mathbb{D}_{F}^{\mu}\right]_{q}=\sum_{\nu} d_{\lambda v}^{\mathbb{C}, e}(q)\left[\mathbb{E}_{F}^{v}: \mathbb{D}_{F}^{\mu}\right]_{q}$.

Proof Let $G^{\lambda}=\left(\left\langle\psi_{\mathfrak{s}}, \psi_{\mathfrak{t}}\right\rangle\right)_{\mathfrak{s}, \mathfrak{t} \in \operatorname{Std}(\mu)}$ be the Gram matrix of the integral graded Specht module $\mathbb{S}^{\lambda}$. Let $N=\# \operatorname{Std}(\boldsymbol{\mu})$ so that $\mathbb{S}_{\mathbb{Z}}^{\lambda}$ is a free $\mathbb{Z}$-module of rank $N$. Since $\mathbb{Z}$ is a principal ideal domain there exist positive integers $d_{1}, \ldots, d_{N}$ such that $d_{1}\left|d_{2}\right| \cdots \mid d_{N}$ and two homogeneous bases $\left\{u_{i}\right\}$ and $\left\{v_{i}\right\}$ of the graded Specht module $\mathbb{S}_{\mathbb{Z}}^{\lambda}$ such that $\left\langle u_{i}, v_{j}\right\rangle=\delta_{i j} d_{i}$, for $1 \leq i, j \leq N$. That is, the diagonal matrix $\operatorname{diag}\left(d_{1}, \ldots, d_{N}\right)$ is the Smith normal form of $G^{\lambda}$. Therefore, $\operatorname{rad} \mathbb{S}_{\mathbb{Z}}^{\lambda}$ is free as an $\mathbb{Z}$-module with basis $\left\{u_{i} \mid d_{i}=0\right\}$ and $\mathbb{E}_{\mathbb{Z}}^{\mu}$ is free as 
a $\mathbb{Z}$-module with basis $\left\{u_{i}+\operatorname{rad} \mathbb{S}_{\mathbb{Z}}^{\lambda} \mid d_{i} \neq 0\right\}$, proving (a). (In the same way, observe that $\left\{v_{i} \mid d_{i}=0\right\}$ is a basis of $\operatorname{rad} \mathbb{S}_{Z}^{\lambda}$ and $\left\{v_{i}+\operatorname{rad} \mathbb{S}_{\mathbb{Z}}^{\lambda} \mid d_{i} \neq 0\right\}$ is a basis of $\mathbb{E}_{\mathbb{Z}}^{\mu}$.)

By definition, $\mathbb{E}_{\mathbb{Q}}^{\mu}=\mathbb{D}_{\mathbb{Q}}^{\mu}$, so part (b) follows from the general theory of (graded) cellular algebras; specifically, see [14, Theorem 2.10]. The same result says that if $F$ is a field then $\mathbb{D}_{F}^{\mu}$ is a self-dual absolutely irreducible graded $\mathscr{R}_{n}^{\Lambda}$-module.

Consider (c). Taking graded characters is exact and commutes with base change, so

$$
\begin{aligned}
& \operatorname{ch}_{q} \mathbb{S}_{F}^{\lambda}=\operatorname{ch}_{q} \mathbb{S}_{\mathbb{Q}}^{\lambda}=\sum_{\nu \in \mathcal{K}_{n}^{\Lambda}} d_{\lambda \nu}^{\mathbb{C}, e}(q) \operatorname{ch}_{q} \mathbb{D}_{\mathbb{Q}}^{\nu}=\sum_{\nu \in \mathcal{K}_{n}^{\Lambda}} d_{\lambda \nu}^{\mathbb{C}, e}(q) \operatorname{ch}_{q} \mathbb{E}_{\mathbb{Q}}^{v} \\
& =\sum_{\boldsymbol{v} \in \mathcal{K}_{n}^{\Lambda}} d_{\lambda \boldsymbol{v}}^{\mathbb{C}, e}(q) \operatorname{ch}_{q} \mathbb{E}_{F}^{v}=\sum_{\boldsymbol{v} \in \mathcal{K}_{n}^{\Lambda}} d_{\lambda \nu}^{\mathbb{C}, e}(q) \sum_{\mu \in \mathcal{K}_{n}^{\Lambda}}\left[\mathbb{E}_{F}^{v}: \mathbb{D}_{F}^{\mu}\right]_{q} \operatorname{ch}_{q} \mathbb{D}_{F}^{\mu} \\
& =\sum_{\mu \in \mathcal{K}_{n}^{\Lambda}}\left(\sum_{\nu \in \mathcal{K}_{n}^{\Lambda}} d_{\lambda \nu}^{\mathbb{C}, e}(q)\left[\mathbb{E}_{F}^{v}: \mathbb{D}_{F}^{\mu}\right]_{q}\right) \operatorname{ch}_{q} \mathbb{D}_{F}^{\mu} .
\end{aligned}
$$

On the other hand, $\operatorname{ch}_{q} \mathbb{S}_{F}^{\lambda}=\sum_{\mu} d_{\lambda, \mu}^{F, e}(q) \mathrm{ch}_{q} \mathbb{D}_{F}^{\mu}$, so part (c) follows by Proposition 2.7.

Let $a_{\boldsymbol{v} \mu}^{F, e}(q)=\left[\mathbb{E}_{F}^{\boldsymbol{v}}: \mathbb{D}_{F}^{\boldsymbol{\mu}}\right]_{q}$, for $\boldsymbol{\mu}, \boldsymbol{v} \in \mathcal{K}_{n}^{\Lambda}$. The matrix $\mathrm{A}_{F, e}(q)=\left(a_{\boldsymbol{v} \boldsymbol{\mu}}^{F, e}(q)\right)_{\boldsymbol{\mu}, \boldsymbol{v} \in \mathcal{K}_{n}^{\Lambda}}$ is the (graded) adjustment matrix of $\mathscr{R}_{n}^{\Lambda}(F)$. Notice that Theorem 2.16(c) is equivalent to the following factorisation of the graded decomposition matrix:

$$
\mathrm{D}_{F, e}(q)=\mathrm{D}_{\mathbb{C}, e}(q) \mathrm{A}_{F, e}(q) .
$$

The adjustment matrix $\mathrm{A}_{F, e}(q)$ coincides with the matrix defined by Brundan and Kleshchev [5, Theorem 5.17] using different arguments. The arguments of [5] amount to carefully choosing a $\mathbb{Z}$-lattice for the simple module $\mathbb{D}_{\mathbb{Q}}^{\mu}$. The proof of Theorem 2.16 shows that the $\psi$-basis, by virtue of Theorem 2.11 , automatically chooses a $\mathbb{Z}$-lattice $\mathbb{E}_{\mathbb{Z}}^{\mu}$ for $\mathbb{D}_{\mathbb{Q}}^{\mu}$.

Corollary 2.18 Suppose that $\lambda \in \mathcal{P}_{n}^{\Lambda}$. Then $\operatorname{ch}_{q} \mathbb{S}_{F}^{\lambda}=\sum_{\mu \in \mathcal{K}_{n}^{\Lambda}} d_{\lambda \mu}^{\mathbb{C}, e}(q) \operatorname{ch}_{q} \mathbb{E}_{F}^{\mu}$.

Let $: \mathbb{Z}\left[q, q^{-1}\right] \longrightarrow \mathbb{Z}\left[q, q^{-1}\right]$ be the unique linear involution such that $\bar{q}=q^{-1}$. Abusing notation, extend this to a map $: \operatorname{Rep} \mathscr{L}_{n} \longrightarrow \operatorname{Rep} \mathscr{L}_{n}$.

Corollary 2.19 Suppose that $\boldsymbol{\mu}, \boldsymbol{v} \in \mathcal{K}_{n}^{\Lambda}$. Then $\overline{\operatorname{ch}_{q} \mathbb{E}_{F}^{v}}=\operatorname{ch}_{q} \mathbb{E}_{F}^{v}$ and $\overline{a_{\boldsymbol{v} \mu}^{F, e}(q)}=a_{\boldsymbol{v} \mu}^{F, e}(q)$.

Proof By construction and Theorem 2.16(b), $\operatorname{ch}_{q} \mathbb{E}_{F}^{v}=\operatorname{ch}_{q} \mathbb{E}_{\mathbb{Q}}^{v}=\operatorname{ch}_{q} \mathbb{D}_{\mathbb{Q}}^{v}$. Therefore, $\overline{\operatorname{ch}_{q} \mathbb{E}_{F}^{v}}=\operatorname{ch}_{q} \mathbb{E}_{F}^{v}$ since $\mathbb{D}_{\mathbb{Q}}^{v}$ is a self-dual graded $\mathscr{R}_{n}^{\Lambda}(\mathbb{Q})$-module. This implies the second claim because

$$
\operatorname{ch}_{q} \mathbb{E}_{F}^{v}=\sum_{\nu \in \mathcal{K}_{n}^{\Lambda}} a_{v \mu}^{F, e}(q) \operatorname{ch}_{q} \mathbb{D}_{F}^{\mu}
$$

and $\mathbb{D}_{F}^{\mu}$ is self-dual.

Let $\boldsymbol{v} \in \mathcal{K}_{n}^{\Lambda}$. Since $\left[\mathbb{E}_{F}^{v}: \mathbb{D}_{F}^{v}\right]_{q}=1$ it follows that $\mathbb{E}_{F}^{v}$ is self-dual if and only if $\mathbb{E}_{F}^{v}=\mathbb{D}_{F}^{v}$. 


\section{Cyclotomic Hecke algebras}

We are now ready to introduce the cyclotomic Hecke algebras of type $A$, and their Specht modules, which are the main focus of this paper. We define several different bases for these algebras and use them to compute the characters of the sum of modules in the Jantzen filtration of the Specht modules.

\subsection{Cyclotomic Hecke algebras of type A}

Let $R$ be a commutative ring with 1 and fix non-negative integers $n, \ell \geq 0$. If $v \in R$ and $k \in \mathbb{Z}$ define the $v$-quantum integer

$$
[k]_{v}= \begin{cases}1+v+\cdots+v^{k-1} & \text { if } k \geq 0 \\ -v^{-1}+v^{-2}-\cdots-v^{-k} & \text { otherwise. }\end{cases}
$$

Observe that if $v \neq 1$ then $[k]_{v}=\left(v^{k}-1\right) /(v-1)$ and if $v=1$ then $[k]_{v}=k$.

We use the following definition of the cyclotomic Hecke algebras of type A, following $[16, \S 2]$.

Definition 3.2 The cyclotomic Hecke algebra of type $A$ with Hecke parameter $v \in R^{\times}$ and $\ell$--charge $\kappa=\left(\kappa_{1}, \ldots, \kappa_{\ell}\right) \in \mathbb{Z}^{\ell}$ is the unital associative $R$-algebra $\mathscr{H}_{n, v}^{\kappa}=\mathscr{H}_{n, v}^{\kappa}(A)$ with generators $T_{1}, \ldots, T_{n-1}, L_{1}, \ldots, L_{n}$ and relations

$$
\begin{aligned}
& \prod_{l=1}^{\ell}\left(L_{1}-\left[\kappa_{l}\right]_{v}\right)=0, \\
& \left(T_{r}-v\right)\left(T_{r}+1\right)=0, \\
& L_{r} L_{t}=L_{t} L_{r}, \\
& T_{r} T_{s}=T_{s} T_{r} \quad \text { if }|r-s|>1, \\
& T_{s} T_{s+1} T_{s}=T_{s+1} T_{s} T_{s+1}, \\
& T_{r} L_{t}=L_{t} T_{r} \quad \text { if } t \neq r, r+1 \text {, } \\
& L_{r+1}=T_{r} L_{r} T_{r}+T_{r},
\end{aligned}
$$

where $1 \leq r<n, 1 \leq s<n-1$ and $1 \leq t \leq n$.

As is explained in $[16, \S 2]$, if $v \neq 1$ these algebras are isomorphic to the Ariki-Koike algebras, which were introduced in [1, §3], and when $v=1$ they are isomorphic to the degenerate Ariki-Koike algebras.

By definition, $\mathscr{H}_{n, v}^{\kappa}$ has a unique anti-isomorphism $*$ that fixes every generator.

Recall from Sect. 2.3 that $\mathfrak{S}_{n}$ is the symmetric group of degree $n$ with distinguished Coxeter generators $s_{1}, \ldots, s_{n-1}$. If $w \in \mathfrak{S}_{n}$ let $L(w)$ be the minimal length of $w$ as a product of the Coxeter generators. As the braid relations hold in $\mathscr{H}_{n}^{\Lambda}$, if $w=s_{i_{1}} \ldots s_{i_{k}}$ is reduced then $T_{w}=T_{i_{1}} \ldots T_{i_{k}} \in \mathscr{H}_{n}^{\Lambda}$ depends only on $w$ and not on the choice of reduced expression. These results are well-known and their proofs can be found, for example, in [33, Chapter 1].

Let $F$ be a field. A non-zero element $\xi \in F$ has quantum characteristic $e$ if $e$ is the smallest positive integer such that $[e] \xi=0$ - set $e=\infty$ if $[k] \xi \neq 0$, for $k>0$. Given $\kappa \in \mathbb{Z}^{\ell}$ define a dominant weight $\Lambda=\Lambda_{\kappa} \in P_{e}^{+}$by using (2.9). Define $\mathscr{H}_{n}^{\Lambda}(F)=\mathscr{H}_{n, \xi}^{\kappa}(F)$. Unravelling the notation, the cyclotomic relation in $\mathscr{H}_{n}^{\Lambda}(F)$ can be written as

$$
0=\prod_{l=1}^{\ell}\left(L_{1}-\left[\kappa_{l}\right]_{\xi}\right)=\prod_{i \in I}\left(L_{1}-[i]_{\xi}\right)^{\left(\Lambda, \alpha_{i}\right)},
$$

where $[i]_{\xi}$ has the obvious meaning, for $i \in I$.

We can now give the relationship between the cyclotomic KLR and Hecke algebras of type $A$. 
Theorem 3.4 (Brundan and Kleshchev [5], Rouquier [38, Theorem 3.12]) Let $F$ be a field. Suppose that $\xi \in F$ has quantum characteristic e and that $\Lambda \in P_{e}^{+}$. Then $\mathscr{H}_{n}^{\Lambda}(F) \cong \mathscr{R}_{n}^{\Lambda}(F)$ as ungraded algebras.

Notice that Theorem 3.4 implies that, up to isomorphism, $\mathscr{H}_{n}^{\Lambda}(F)$ depends only on $e, \Lambda$ and $F$, and not on the particular choice of $\xi \in F$ with quantum characteristic $e$. As explained in Sect. 3.3 below, we will always assume that $e$ is finite.

\subsection{The Murphy basis}

Following [10, §3], this section defines a cellular basis of $\mathscr{H}_{n}^{\Lambda}$ that we will use to define Specht modules for $\mathscr{H}_{n}^{\Lambda}$.

Let $\lambda \in \mathcal{P}_{n}^{\Lambda}$ and define elements

$$
m_{\lambda}=u_{\lambda} \sum_{w \in \mathfrak{S}_{\lambda}} T_{w}, \quad \text { where } \quad u_{\lambda}=\prod_{l=2}^{\ell} \prod_{m=1}^{\left|\lambda^{(1)}\right|+\cdots+\left|\lambda^{(l-1)}\right|}\left(L_{m}-\left[\kappa_{l}\right]\right) .
$$

For $\mathfrak{s}, \mathfrak{t} \in \operatorname{Std}(\lambda)$ define $m_{\mathfrak{s t}}=T_{d(\mathfrak{s})}^{*} m_{\lambda} T_{d(\mathfrak{t})}$. By definition, $m_{\mathfrak{s t}}^{*}=m_{\mathfrak{t} \mathfrak{s}}$.

Theorem 3.5 ( [10, Theorem 3.26] and [2, Theorem 6.3]) For any ring $R, \mathscr{H}_{n}^{\Lambda}$ is an $R$-free cellular algebra with cellular basis $\left\{m_{\mathfrak{s t}} \mid \mathfrak{s}, \mathfrak{t} \in \operatorname{Std}(\lambda)\right.$ for $\left.\lambda \in \mathcal{P}_{n}^{\Lambda}\right\}$.

The two papers [10] and [2] prove this result in cases where $\xi \neq 1$ and $\xi=1$, respectively. Hence, exactly as before (except that we no longer have a grading), for each $\ell$-partition $\lambda \in \mathcal{P}_{n}^{\Lambda}$ we have a Specht module $S^{\lambda}$, which is a left $\mathscr{H}_{n, v^{\kappa}}$-module.

As in Sect. 2.2, for each $\lambda \in \mathcal{P}_{n}^{\Lambda}$ let $S^{\lambda}$ be the Specht module determined by the cellular basis of Theorem 3.5. Then $S^{\lambda}$ is free as an $R$-module with basis $\left\{m_{\mathfrak{t}} \mid \mathfrak{t} \in \operatorname{Std}(\lambda)\right\}$ and it comes equipped with an inner product $\langle$,$\rangle that is uniquely determined by$

$$
m_{\mathfrak{s}} m_{\mathfrak{t} \mathfrak{v}}=\left\langle m_{\mathfrak{s}}, m_{\mathfrak{t}}\right\rangle m_{\mathfrak{v}}, \quad \text { for } \mathfrak{s}, \mathfrak{t}, \mathfrak{v} \in \operatorname{Std}(\lambda) .
$$

Recall from (2.15) that $d_{\lambda \mu}^{F, e}(q)=\left[\mathbb{S}_{F}^{\lambda}: \mathbb{D}_{F}^{\mu}\right]_{q}$ is a graded decomposition number of $\mathscr{R}_{n}^{\Lambda}(F)$, for $\lambda \in \mathcal{P}_{n}^{\Lambda}$ and $\mu \in \mathcal{K}_{n}^{\Lambda}$. By [6, Theorem 4.10], or [14, §5.2], under the isomorphism of Theorem 3.4 the modules $S_{F}^{\lambda}$ and $\mathbb{S}_{F}^{\lambda}$ coincide, once we forget the grading. In more detail, we have:

Corollary 3.6 Let $\lambda \in \mathcal{P}_{n}^{\Lambda}$ and $\boldsymbol{\mu} \in \mathcal{K}_{n}^{\Lambda}$. Then $S_{F}^{\lambda} \cong \underline{\mathbb{S}}_{F}^{\lambda}$ and $D_{F}^{\mu} \cong \underline{\mathbb{D}}_{F}^{\mu}$. Consequently,

$$
\left[S_{F}^{\lambda}: D_{F}^{\mu}\right]=d_{\lambda \mu}^{F, e}(1) .
$$

Let $\mathrm{D}_{F, e}=\left(d_{\lambda \mu}^{F, e}\right)$ be the decomposition matrix of $\mathscr{H}_{n}^{\Lambda}(F)$. Then $\mathrm{D}_{F, e}=\mathrm{D}_{F, e}(1)$ by Corollary 3.6, where $\mathrm{D}_{F, e}(1)=\left.\mathrm{D}_{F, e}(q)\right|_{q=1}$ is the graded decomposition matrix of Sect. 2.3 evaluated at $q=1$.

\subsection{Seminormal forms}

This section recalls the construction of the seminormal basis of $\mathscr{H}_{n}^{\Lambda}(\mathcal{K})$ in the semisimple case, so all of these results in this section, ultimately, go back to the seminal work of Young [45]. We follow the general framework from [35] but see also [1,16,34]. 
For the rest of this paper we assume that $e<\infty$ in Definition 2.5 or, equivalently, that $\xi$ is not a root of unity in Definition 3.2. By [16, Corollary 2.10], up to isomorphism, we can always assume that $e$ is finite, so there is no loss of generality in making this assumption. The advantage of assuming that $e$ is finite is that this is allows us to adjust the multicharge of (2.9) modulo $e$ in (3.7) below.

Let $F$ be a field. As in (2.9) we fix an $\ell$-charge $\kappa=\left(\kappa_{1}, \ldots, \kappa_{\ell}\right) \in \mathbb{Z}^{\ell}$. For the rest of this paper, we impose the additional assumption that

$$
\kappa_{1}-2(\ell-1) n \geq \kappa_{2}-2(\ell-2) n \geq \cdots \geq \kappa_{\ell-1}-2 n \geq \kappa_{\ell} \geq n .
$$

Implicitly, this assumption fixes a choice of lattice in a modular system for $\mathscr{H}_{n}^{\Lambda}(F)$ and, in principle, the Jantzen filtrations that we construct below depend on the choices in (3.7). In practice, we need (3.7) for the results on seminormal forms that we use below because (3.7) ensures that standard tableaux are uniquely determined by their content sequences, which are defined below. This said, assumption (3.7) is only a technical convenience because, by (2.9), the dominant weight $\Lambda=\Lambda_{\kappa}$, and hence the algebra $\mathscr{H}_{n}^{\Lambda}(F)$, depend only on the multiset $\left\{\bar{\kappa}_{1}, \ldots, \bar{\kappa}_{\ell}\right\}$. In particular, $\mathscr{H}_{n}^{\Lambda}(F)$ does not depend on (3.7).

As above, consider the cyclotomic Hecke algebra $\mathscr{H}_{n}^{\Lambda}=\mathscr{H}_{n}^{\Lambda}(F)$ with Hecke parameter $\xi \in F$ of quantum characteristic $e<\infty$ and cyclotomic parameters $\left[\kappa_{m}\right]_{\xi}$, for $1 \leq m \leq \ell$, where $\kappa=\left(\kappa_{1}, \ldots, \kappa_{\ell}\right) \in \mathbb{Z}^{\ell}$ is a fixed choice of $\ell$-charge.

Let $x$ be an indeterminate over $F$ and consider the localisation $\mathcal{O}=F[x]_{(x)}$ of the polynomial ring $F[x]$ at the prime ideal $(x)=x F[x]$. Let $\mathcal{K}=F(x)$ be the field of fractions of $F[x]$. Let $\mathscr{H}_{n}^{\Lambda}(\mathcal{O})$ be the cyclotomic Hecke algebra over $\mathcal{O}$ with Hecke parameter $z=x+\xi$ and with cyclotomic parameters $\left(\left[\kappa_{1}\right]_{z}, \ldots,\left[\kappa_{\ell}\right]_{z}\right)$. Let $\mathscr{H}_{n}^{\Lambda}(\mathcal{K})=\mathscr{H}_{n}^{\Lambda}(\mathcal{O}) \otimes_{\mathcal{O}} \mathcal{K}$. Then $\mathscr{H}_{n}^{\Lambda}(\mathcal{K})$ is a split semisimple algebra. Consider the field $F$ as an $\mathcal{O}$-algebra where $x$ acts as 0 . Then $\mathscr{H}_{n}^{\Lambda}(F) \cong \mathscr{H}_{n}^{\Lambda}(\mathcal{O}) \otimes_{\mathcal{O}} F$.

For $\lambda \in \mathcal{P}_{n}^{\Lambda}$ let $S_{\mathcal{O}}^{\lambda}$ be the (ungraded) Specht module for $\mathscr{H}_{n}^{\Lambda}(\mathcal{O})$ determined by the $\left\{m_{\mathfrak{s t}}\right\}$ cellular basis and $\lambda$. Then $S_{\mathcal{K}}^{\lambda}=S_{\mathcal{O}}^{\lambda} \otimes_{\mathcal{O}} \mathcal{K}$ is the Specht module for $\mathscr{H}_{n}^{\Lambda}(\mathcal{K})$.

Let $\mathfrak{t}=\left(\mathfrak{t}^{(1)}|\ldots| \mathfrak{t}^{(\ell)}\right) \in \operatorname{Std}(\lambda)$, for $\lambda \in \mathcal{P}_{n}^{\Lambda}$. Suppose that the integer $m$, with $1 \leq m \leq n$, appears in row $r$ and column $c$ of $\mathfrak{t}^{(k)}$. The content of $m$ in $\mathfrak{t}$ is $c_{m}(\mathfrak{t})=\kappa_{m}+c-r$. Observe that the residue of $m$ is $r_{m}(\mathfrak{t})=c_{m}(\mathfrak{t})+e \mathbb{Z} \in I$. The content sequence of $\mathfrak{t}$ is $c(\mathfrak{t})=$ $\left(c_{1}(\mathfrak{t}), \ldots, \mathrm{c}_{n}(\mathfrak{t})\right)$. More generally, the content of the node $(m, r, c)$ is $\mathrm{c}(m, r, c)=\kappa_{m}+c-r$. The key point of these definitions is that $\mathfrak{s}=\mathfrak{t}$ if and only if $\mathrm{c}(\mathfrak{s})=\mathrm{c}(\mathfrak{t})$, for $\mathfrak{s}, \mathfrak{t} \in \operatorname{Std}\left(\mathcal{P}_{n}^{\Lambda}\right)$. This is easily proved by induction on $n$ using (3.7). ${ }^{1}$

James and the author [19, Proposition 3.7] proved the following fundamental property of this basis:

$$
m_{\mathfrak{s}} L_{k}=\left[c_{k}(\mathfrak{s})\right] m_{\mathfrak{s}}+\sum_{\mathfrak{u} \triangleright \mathfrak{s}} r_{\mathfrak{u}} m_{\mathfrak{u}}, \quad \text { for } \mathfrak{s} \in \operatorname{Std}(\lambda), 1 \leq k \leq n \text { and some } r_{\mathfrak{u}} \in R . \text { (3.8) }
$$

Let $\mathfrak{s}, \mathfrak{t} \in \operatorname{Std}(\lambda)$, for $\lambda \in \mathcal{P}_{n}^{\Lambda}$. Define

$$
f_{\mathfrak{s t}}=F_{\mathfrak{s}} m_{\mathfrak{s t}} F_{\mathfrak{t}}, \quad \text { where } \quad F_{\mathfrak{t}}=\prod_{k=1}^{n} \prod_{\substack{\mathfrak{s} \in \operatorname{Std}\left(\mathcal{P}_{n}^{\Lambda}\right) \\ c_{k}(\mathfrak{s}) \neq c_{k}(\mathfrak{t})}} \frac{L_{k}-\left[\mathbf{c}_{k}(\mathfrak{s})\right]_{z}}{\left[\mathbf{c}_{k}(\mathfrak{t})\right]_{z}-\left[\mathbf{c}_{k}(\mathfrak{s})\right]_{z}}
$$

\footnotetext{
1 This is the only place where assumption (3.7) is needed in the construction of the seminormal basis. That is, in this Sect. (3.7) is only used to ensure that the content sequences separate the standard tableaux. The stronger form of (3.7) is used to define sequences of charged beta numbers in Sect. 5.
} 
Equation (3.8) implies that

$$
f_{\mathfrak{s t}} \equiv m_{\mathfrak{s t}}+\sum_{\mathfrak{u} \triangleright \mathfrak{s}, \mathfrak{v} \triangleright \mathfrak{t}} r_{\mathfrak{u v}} m_{\mathfrak{u v}}\left(\bmod \mathscr{H}_{n}^{\triangleright \lambda}\right), \quad \text { for some } r_{\mathfrak{u v}} \in \mathcal{K}
$$

In particular, $\left\{f_{\mathfrak{s t}}\right\}$ is a basis of $\mathscr{H}_{n}^{\Lambda}(\mathcal{K})$. Since the transition matrix between the two bases $\left\{m_{\mathfrak{s t}}\right\}$ and $\left\{f_{\mathfrak{s t}}\right\}$ is unitriangular it is not hard to see that $\left\{f_{\mathfrak{s t}}\right\}$ is a cellular basis of $\mathscr{H}_{n}^{\Lambda}(\mathcal{K})$. Moreover, if $\lambda \in \mathcal{P}_{n}^{\Lambda}$ and $\mathfrak{s}, \mathfrak{t} \in \operatorname{Std}(\lambda)$ then $S_{\mathcal{K}}^{\lambda} \cong f_{\mathfrak{s t}} \mathscr{H}_{n}^{\Lambda}(\mathcal{K})$.

In fact, $\left\{f_{\mathfrak{s t}}\right\}$ is a seminormal basis of $\mathscr{H}_{n}^{\Lambda}(\mathcal{K})$ in the sense of [16, Definition 3.4], which means that $f_{\mathfrak{s t}}$ are simultaneous eigenvectors for $L_{1}, \ldots, L_{n}$. Explicitly, in view of [19, Proposition 3.7],

$$
L_{k} f_{\mathfrak{s t}}=\left[\mathrm{c}_{k}(\mathfrak{s})\right] f_{\mathfrak{s t}} \quad \text { and } \quad f_{\mathfrak{s t}} L_{k}=\left[\mathrm{c}_{k}(\mathfrak{t})\right] f_{\mathfrak{s t}}, \quad \text { for } 1 \leq k \leq n .
$$

These two formulas are equivalent since $f_{\mathfrak{s t}}^{*}=f_{\mathfrak{t s}}$. Using (3.10) and the definitions it follows that $F_{\mathfrak{u}} f_{\mathfrak{s t}} F_{\mathfrak{v}}=\delta_{\mathfrak{s u}} \delta_{\mathfrak{t v}} f_{\mathfrak{s t}}$ and hence that there exist scalars $\gamma_{\mathfrak{t}} \in \mathcal{K}$ such that

$$
f_{\mathfrak{s t}} f_{\mathfrak{u v}}=\delta_{\mathfrak{t u}} \gamma_{\mathfrak{t}} f_{\mathfrak{s v}}, \quad \text { for all } \mathfrak{s}, \mathfrak{t} \in \operatorname{Std}(\boldsymbol{\lambda}) \text { and } \mathfrak{u}, \mathfrak{v} \in \operatorname{Std}(\boldsymbol{\mu}) .
$$

In particular, it follows that $F_{\mathfrak{t}}=\frac{1}{\gamma_{\mathfrak{t}}} f_{\mathfrak{t t}}$, for all $\mathfrak{t} \in \operatorname{Std}\left(\mathcal{P}_{n}^{\Lambda}\right)$.

Suppose that $\mathfrak{s}, \mathfrak{t v} \in \operatorname{Std}(\lambda)$ and that $\mathfrak{v}=\mathfrak{t}(r, r+1)$. Then by [16, Theorem 3.9 and Proposition 3.11],

$$
f_{\mathfrak{s t}} T_{r}= \begin{cases}f_{\mathfrak{s} \mathfrak{v}}-\frac{1}{\left[\rho_{r}(\mathfrak{t})\right]} f_{\mathfrak{s t}}, & \text { if } \mathfrak{t} \triangleright \mathfrak{v}, \\ \frac{\left[1+\rho_{r}(\mathfrak{t})\right]\left[1-\rho_{r}(\mathfrak{t})\right]}{\left[\rho_{r}(\mathfrak{t})\right]^{2}} f_{\mathfrak{s v}}-\frac{1}{\left[\rho_{r}(\mathfrak{t})\right]} f_{\mathfrak{s t}}, & \text { if } \mathfrak{v} \triangleright \mathfrak{t},\end{cases}
$$

where $\rho_{r}(\mathfrak{t})=\mathrm{c}_{r}(\mathfrak{t})-\mathrm{c}_{r+1}(\mathfrak{t})$. Using this to compute $\gamma_{\mathfrak{v}} f_{\mathfrak{v} \mathfrak{v}}=f_{\mathfrak{v} \mathfrak{v}} f_{\mathfrak{v} \mathfrak{v}}$, when $\mathfrak{s}=\mathfrak{v}$, shows that:

Corollary 3.12 [16, Corollary 3.10] Suppose that $\mathfrak{t}, \mathfrak{v} \in \operatorname{Std}(\lambda), \mathfrak{t} \triangleright \mathfrak{v}$ and that $\mathfrak{v}=\mathfrak{t}(r, r+1)$, for some $r$ with $1 \leq r<n$. Then

$$
\gamma_{\mathfrak{v}}=\frac{\left[1+\rho_{r}(\mathfrak{t})\right]\left[1-\rho_{r}(\mathfrak{t})\right]}{\left[\rho_{r}(\mathfrak{t})\right]^{2}} \gamma_{\mathfrak{t}} .
$$

\subsection{Idempotents and characters}

In view of Theorem 3.4, over a field the cyclotomic Hecke algebra $\mathscr{H}_{n}^{\Lambda}$ has analogues of the KLR idempotents $e(\mathbf{i}) \in \mathscr{R}_{n}^{\Lambda}$, for $\mathbf{i} \in I^{n}$. Rather than working over a field, we need these idempotents over $\mathcal{O}$. Fortunately, these idempotents are easy to describe using the seminormal basis. For $\mathbf{i} \in I^{n}$ define

$$
f_{\mathbf{i}}=\sum_{\mathfrak{t} \in \operatorname{Std}_{\mathbf{i}}\left(\mathcal{P}_{n}^{\Lambda}\right)} F_{\mathfrak{t}}=\sum_{\mathfrak{t} \in \operatorname{Std}_{\mathbf{i}}\left(\mathcal{P}_{n}^{\Lambda}\right)} \frac{1}{\gamma_{\mathfrak{t}}} f_{\mathfrak{t t}} .
$$

By definition, $f_{\mathbf{i}} \in \mathscr{H}_{n}^{\Lambda}(\mathcal{K})$. In fact, inspired by results of Murphy, we have:

Lemma 3.13 Suppose that $\mathbf{i} \in I^{n}$. Then $f_{\mathbf{i}}=f_{\mathbf{i}}^{*}$ is an idempotent in $\mathscr{H}_{n}^{\Lambda}(\mathcal{O})$. Moreover, if $\mathbf{j} \in I^{n}$ then $f_{\mathbf{i}} f_{\mathbf{j}}=\delta_{\mathbf{i j}} f_{\mathbf{i}}$. 
Proof First observe that if $\mathfrak{t}$ is a standard tableau then $F_{\mathfrak{t}}^{*}=F_{\mathfrak{t}}$ since, by definition, $L_{k}^{*}=L_{k}$ for $1 \leq k \leq n$. Therefore, $f_{\mathbf{i}}^{*}=f_{\mathbf{i}}$, for all $\mathbf{i} \in I^{n}$.

By [16, Lemma 4.3], $f_{\mathbf{i}} \in \mathscr{H}_{n}^{\Lambda}(\mathcal{O})$. In order to apply this lemma from [16] we need to first show that $(\mathcal{O}, t)$ is an "idempotent subring of $\mathcal{K}$ " in the sense of [16, Definition 4.1]. In fact, there is nothing to do here because this is already established in [16, Example 4.2(b)].

Finally, $f_{\mathbf{i}} f_{\mathbf{j}}=\delta_{\mathbf{i j}} f_{\mathbf{i}}$ because $\left\{F_{\mathfrak{t}} \mid \mathfrak{t} \in \operatorname{Std}\left(\mathcal{P}_{n}^{\Lambda}\right)\right\}$ is a set of pairwise orthogonal idempotents.

Remark 3.14 By definition, $f_{\mathbf{i}} \neq 0$ if and only if $\mathbf{i}=r(\mathfrak{t})$ for some standard tableau $\mathfrak{t} \in$ $\operatorname{Std}\left(\mathcal{P}_{n}^{\Lambda}\right)$. In contrast, it is not clear from Definition 2.5 when the KLR idempotent $e(\mathbf{i})$ is non-zero. In fact, in view of Theorem $2.11, e(\mathbf{i}) \neq 0$ if and only if $f_{\mathbf{i}} \neq 0$.

Since $\mathscr{H}_{n}^{\Lambda} \cong \mathscr{H}_{n}^{\Lambda}(\mathcal{O}) \otimes_{\mathcal{O}} F$ we obtain pairwise orthogonal idempotents $f_{\mathbf{i}} \otimes 1$ in $\mathscr{H}_{n}^{\Lambda}$. We abuse notation and write $f_{\mathbf{i}}$ for these idempotents in both $\mathscr{H}_{n}^{\Lambda}(\mathcal{O})$ and $\mathscr{H}_{n}^{\Lambda}$. The meaning should always be clear from context.

As a first consequence of the existence of the idempotents $f_{\mathbf{i}}$ in $\mathscr{H}_{n}^{\Lambda}(\mathcal{O})$ and $\mathscr{H}_{n}{ }^{\Lambda}$, we can define an analogue of the graded characters of Definition 2.6 for these algebras. If $M$ is an (ungraded) $\mathscr{H}_{n}^{\Lambda}$-module then $M=\bigoplus_{\mathbf{i}} M_{\mathbf{i}}$ as an $\mathcal{O}$-module, when $M_{\mathbf{i}}=M f_{\mathbf{i}}$.

Definition 3.15 Let $M$ be an $\mathscr{H}_{n}^{\Lambda}(F)$-module. the character of $M$ is

$$
\operatorname{ch} M=\sum_{\mathbf{i} \in I^{n}}\left(\operatorname{dim} M_{\mathbf{i}}\right) \mathbf{i} \in \mathbb{Z}\left[I^{n}\right] .
$$

Just as with the graded character $\mathrm{ch}_{q}$, we can view ch as an exact functor from the category of $\mathscr{H}_{n}^{\Lambda}$-modules to the category of $\mathscr{L}_{n}^{\prime}$-modules, where $\mathscr{L}_{n}^{\prime}=\left\langle L_{1}, \ldots, L_{n}\right\rangle$. Let $\operatorname{Rep} \mathscr{H}_{n} \Lambda$ be the Grothendieck group of finitely generated $\mathscr{H}_{n}^{\Lambda}$-modules. Then we can view ch as the induced map

$$
\text { ch }: \operatorname{Rep} \mathscr{H}_{n}^{\Lambda} \longrightarrow \operatorname{Rep} \mathscr{L}_{n}^{\prime} \hookrightarrow \mathbb{Z}\left[I^{n}\right] .
$$

Like the graded characters and Proposition 2.7, we have:

Proposition 3.16 Let $F$ be a field. Then the character map ch $: \operatorname{Rep} \mathscr{R}_{n}^{\Lambda}(F) \longrightarrow \operatorname{Rep} \mathbb{Z}\left[I^{n}\right]$ is injective.

Proof This is part of the folklore for $\mathscr{H}_{n}^{\Lambda}$. It is proved in exactly the same way as Proposition 2.7 using the corresponding results for the affine Hecke algebra; see, for example, [27, Theorem 3.3.1].

Finally, note that if $M$ is a graded $\mathscr{R}_{n}^{\Lambda}(F)$-module then, in view of Theorem $3.4, \underline{M}$ is naturally an $\mathscr{H}_{n}^{\Lambda}(F)$-module. The proof of Theorem 3.4 (or, more concretely, [16, Theorem A]), identifies the KLR idempotent $e(\mathbf{i}) \in \mathscr{R}_{n}^{\Lambda}(F)$ with $f_{\mathbf{i}} \in \mathscr{H}_{n}^{\Lambda}(F)$, for all $\mathbf{i} \in I^{n}$. Therefore, $\operatorname{ch} \underline{M}=\left.\operatorname{ch}_{q} M\right|_{q=1}$. Hence, Proposition 3.16 implies Proposition 2.7.

\subsection{Gram determinants of Specht modules}

In this section we compute the Gram determinant of the Specht module with respect to the Murphy basis. Gram determinants are only well-defined up to a sign that depends on the ordering of the rows and columns. In this and later sections we fix a total ordering of the tableaux in $\operatorname{Std}(\lambda)$ that refines the dominance ordering and we use this ordering for both the 
rows and the columns of the Gram matrices. In fact, Corollary 3.18 below implies that if the same ordering is used for the rows and columns of the Gram matrix then the (sign of the) Gram determinant is independent of this choice of ordering.

Throughout this section fix an $\ell$-partition $\lambda \in \mathcal{P}_{n}^{\Lambda}$. The Gram matrix of the Specht module $S_{\mathcal{K}}^{\lambda}$ is

$$
G^{\lambda}=\left(\left\langle m_{\mathfrak{s}}, m_{\mathfrak{t}}\right\rangle\right)_{\mathfrak{s}, \mathfrak{t} \in \operatorname{Std}(\lambda)} .
$$

For $\mathfrak{t} \in \operatorname{Std}(\lambda)$ define $f_{\mathfrak{t}}=m_{\mathfrak{t}} F_{\mathfrak{t}}$. Then $f_{\mathfrak{t}}=m_{\mathfrak{t}}+\sum_{\mathfrak{u} \triangleright \mathfrak{t}} s_{\mathfrak{u}} m_{\mathfrak{u}}$ by (3.9), for some $s_{\mathfrak{u}} \in \mathcal{K}$. Therefore, $\left\{f_{\mathfrak{t}} \mid \mathfrak{t} \in \operatorname{Std}(\lambda)\right\}$ is a "seminormal" basis of $S_{\mathcal{K}}^{\lambda}$.

The following result is essentially a restatement of [19, Proposition 3.19], although the proof below is considerably easier because it exploits the defining property of the inner product on a cell module.

Lemma 3.17 Suppose that $\mathfrak{s}, \mathfrak{t} \in \operatorname{Std}(\lambda)$, for $\lambda \in \mathcal{P}_{n}^{\Lambda}$. Then $\left\langle f_{\mathfrak{s}}, f_{\mathfrak{t}}\right\rangle=\delta_{\mathfrak{s t}} \gamma_{\mathfrak{t}}$.

Proof In view of (2.2) and (3.11), $\left\langle f_{\mathfrak{s}}, f_{\mathfrak{t}}\right\rangle f_{\mathfrak{t}}=f_{\mathfrak{s}} f_{\mathfrak{t t}}=\delta_{\mathfrak{s t}} \gamma_{\mathfrak{s}} f_{\mathfrak{s}}$. Hence, $\left\langle f_{\mathfrak{s}}, f_{\mathfrak{t}}\right\rangle=\delta_{\mathfrak{s t}} \gamma_{\mathfrak{t}}$ as claimed.

The transition matrix between the Murphy and the seminormal bases of $S_{\mathcal{K}}^{\lambda}$ is unitriangular, so:

Corollary 3.18 Suppose that $\lambda \in \mathcal{P}_{n}^{\Lambda}$. Then $\operatorname{det} G^{\lambda}=\operatorname{det}\left(\left\langle f_{\mathfrak{s}}, f_{\mathfrak{t}}\right\rangle\right)=\prod_{\mathfrak{t} \in \operatorname{Std}(\lambda)} \gamma_{\mathfrak{t}}$.

The Gram determinant is quite a crude statistic. To approach the Jantzen sum formula we need to embellish det $G^{\lambda}$ by adding enough data so that it determines a formal character. To do this we need a new cellular basis of $\mathscr{H}_{n}^{\Lambda}$.

Let $\mathfrak{s}, \mathfrak{t} \in \operatorname{Std}(\boldsymbol{\lambda})$ and set $\mathbf{i}=\mathrm{r}(\mathfrak{s})$ an $\mathbf{j}=\mathrm{r}(\mathfrak{t})$. Define $b_{\mathfrak{s t}}=f_{\mathbf{i}} m_{\mathfrak{s t}} f_{\mathbf{j}}$. We can view $b_{\mathfrak{s t}}$ as an element of $\mathscr{H}_{n}^{\Lambda}(\mathcal{O})$ or of $\mathscr{H}_{n}^{\Lambda}$. By Lemma $3.13, b_{\mathfrak{s t}}=f_{\mathbf{i}} b_{\mathfrak{s t}} f_{\mathbf{j}}$, where $\mathbf{i}=\mathrm{r}(\mathfrak{s})$ and $\mathbf{j}=r(\mathfrak{t})$.

Proposition 3.19 The $\operatorname{set}\left\{b_{\mathfrak{s t}} \mid \mathfrak{s}, \mathfrak{t} \in \operatorname{Std}(\lambda), \lambda \in \mathcal{P}_{n}^{\Lambda}\right\}$ is a cellular basis of $\mathscr{H}_{n}^{\Lambda}(\mathcal{O})$.

Proof By (3.8), if $\mathfrak{u} \in \operatorname{Std}_{\mathbf{i}}\left(\mathcal{P}_{n}^{\Lambda}\right)$ then there exist scalars $r_{\mathfrak{t u v}} \in \mathcal{O}$ such that

$$
m_{\mathfrak{s t}} F_{\mathfrak{u}} \equiv \delta_{\mathfrak{t u}} m_{\mathfrak{s t}}+\sum_{\mathfrak{v} \triangleright \mathfrak{t}} r_{\mathfrak{t u v}} m_{\mathfrak{s v}}\left(\bmod \mathscr{H}_{n}^{\triangleright \lambda}\right)
$$

Hence, $m_{\mathfrak{s t}} f_{\mathbf{i}}$ is equal to $m_{\mathfrak{s t}}$ plus a linear combination of more dominant terms, so the transition matrix from the basis $\left\{m_{\mathfrak{s t}}\right\}$ to the set $\left\{b_{\mathfrak{s t}}\right\}$ is unitriangular. In particular, $\left\{b_{\mathfrak{s t}}\right\}$ is a basis of $\mathscr{H}_{n}^{\Lambda}(\mathcal{O})$. It is now straightforward to check that $\left\{b_{\mathfrak{s t}}\right\}$ is a cellular basis of $\mathscr{H}_{n}^{\Lambda}(\mathcal{O})$. As the cellularity of this basis is not used in what follows we leave these details to the reader.

Remark 3.20 The $b$-basis is compatible with the block decomposition of $\mathscr{H}_{n}^{\Lambda}$. This basis first appeared in a more general context in [35, Theorem 4.5]. Instead of the $b$-basis we could use an analogue of the $\psi$-basis from Theorem 2.11 for $\mathscr{H}_{n}^{\Lambda}(\mathcal{O})$, which is constructed in $[16, \S 4]$. There is no real gain in doing this, however, because the $\psi$-basis of $\mathscr{H}_{n}^{\Lambda}(\mathcal{O})$ takes considerably more effort to define. 
The $b$-basis $\left\{b_{\mathfrak{s t}}\right\}$ of $\mathscr{H}_{n}^{\Lambda}$ gives a $b$-basis $\left\{b_{\mathfrak{t}}\right\}$ of the Specht module $S_{\mathcal{O}}^{\lambda}$ and, by extension of scalars, a $b$-basis of $S_{\mathcal{K}}^{\lambda}$. The easiest way to see this is to notice that $b_{\mathfrak{t}^{\lambda}}=m_{\mathfrak{t}^{\lambda}}$, so that $b_{\mathfrak{t}^{\lambda} \mathfrak{t}}=\sum_{\mathfrak{v} \unrhd \mathfrak{t}} a_{\mathfrak{t v}} m_{\mathfrak{t}^{\lambda} \mathfrak{v}}$ for some $a_{\mathfrak{t v}} \in \mathcal{O}$ by the proof of Proposition 3.19. Hence, we can set $b_{\mathfrak{t}}=\sum_{\mathfrak{v}} a_{\mathfrak{u v}} m_{\mathfrak{v}} \in S^{\lambda}$, for $\mathfrak{t} \in \operatorname{Std}(\lambda)$. For each $\mathbf{i} \in I^{n}$ define Gram matrices:

$$
G_{\mathbf{i}}^{\lambda}=\left(\left\langle b_{\mathfrak{s}}, b_{\mathfrak{t}}\right\rangle\right)_{\mathfrak{s}, \mathfrak{t} \in \operatorname{Std}_{\mathbf{i}}(\lambda)} .
$$

We adopt the convention that $\operatorname{det} G_{\mathbf{i}}^{\lambda}=1$ if $\operatorname{Std}_{\mathbf{i}}(\lambda)=\emptyset$. Then we have:

Lemma 3.21 Let $\lambda \in \mathcal{P}_{n}^{\Lambda}$. Then $\operatorname{det} G^{\lambda}=\prod_{\mathbf{i} \in I^{n}} \operatorname{det} G_{\mathbf{i}}^{\lambda}$. Moreover, if $\mathbf{i} \in I^{n}$ then

$$
\operatorname{det} G_{\mathbf{i}}^{\lambda}=\prod_{\mathfrak{t} \in \operatorname{Std}_{\mathbf{i}}(\lambda)} \gamma_{\mathfrak{t}} .
$$

Proof The transition matrix from the Murphy basis to the $b$-basis is unitriangular, so det $G^{\lambda}=$ $\operatorname{det}\left(\left\langle b_{\mathfrak{s}}, b_{\mathfrak{t}}\right\rangle\right)$. Next, observe that if $\mathfrak{s} \in \operatorname{Std}_{\mathbf{i}}(\lambda)$ and $\mathfrak{t} \in \operatorname{Std}_{\mathbf{j}}(\lambda)$ then

$$
\left\langle b_{\mathfrak{s}}, b_{\mathfrak{t}}\right\rangle=\left\langle b_{\mathfrak{s}} f_{\mathbf{i}}, b_{\mathfrak{t}} f_{\mathbf{j}}\right\rangle=\left\langle b_{\mathfrak{s}}, b_{\mathfrak{t}} f_{\mathbf{j}} f_{\mathbf{i}}^{*}\right\rangle=\delta_{\mathbf{i j}}\left\langle b_{\mathfrak{s}}, b_{\mathfrak{t}}\right\rangle,
$$

where the last equality follows by Lemma 3.13 . Hence, $\operatorname{det} G^{\lambda}=\prod_{\mathbf{i} \in I^{n}} \operatorname{det} G_{\mathbf{i}}^{\lambda}$. Finally,

$$
\operatorname{det} G_{\mathbf{i}}^{\lambda}=\left(\left\langle f_{\mathfrak{s}}, f_{\mathfrak{t}}\right\rangle\right)=\prod_{\mathfrak{t} \in \operatorname{Std}_{\mathbf{i}}(\lambda)} \gamma_{\mathfrak{t}}
$$

because, by going through the Murphy basis, the transition matrix from the $b$-basis to the seminormal basis of $S_{\mathcal{K}}^{\lambda}$ is unitriangular.

\section{Jantzen characters}

Jantzen filtrations of Specht modules underpin all of the results of this paper. Extending what we said in the introduction, Jantzen filtrations can be defined whenever we are given a module over a principal ideal domain that has a non-degenerate bilinear form. Therefore, to define the Jantzen filtrations of the Specht modules we need to work in the ungraded setting because the form on the graded Specht module $\mathbb{S}_{\mathbb{Q}}^{\mu}$ is degenerate unless $\mathbb{S}_{\mathbb{Q}}^{\mu}=\mathbb{D}_{\mathbb{Q}}^{\mu}$, as is evident from Theorem 2.16. On the other hand, we need to incorporate the idempotents $e(\mathbf{i})$ from Sect. 2.3 into the Jantzen filtrations, which requires a careful choice of ground-ring. This section sets up the necessary machinery and then proves our main results.

\subsection{Jantzen filtrations}

We recall Jantzen's construction of filtrations of modules that come equipped with a nondegenerate bilinear form in the special case when the ground ring is $F[x]_{(x)}$. We then apply Jantzen's construction to the Specht modules of $\mathscr{H}_{n}^{\Lambda}(F)$.

As above, let $\mathcal{O}=F[x]_{(x)}$ and let $\mathcal{K}=F(x)$. Let $M_{\mathcal{O}}$ be a free $\mathcal{O}$-module of finite rank and let $M_{\mathcal{K}}=M_{\mathcal{O}} \otimes_{\mathcal{O}} \mathcal{K}$. Suppose that there exists a non-degenerate bilinear form

$$
\langle,\rangle: M_{\mathcal{O}} \times M_{\mathcal{O}} \longrightarrow \mathcal{O} .
$$

By definition, $\mathcal{O}$ is a discrete valuation ring with maximal ideal $x \mathcal{O}$. Let $v_{x}$ be the associated valuation. Explicitly, if $0 \neq a \in \mathcal{O}$ then $v_{x}(a)=\max \left\{k \geq 0 \mid a \in x^{k} \mathcal{O}\right\}$. In particular, $v_{x}(x+\xi)=0$. 
The Jantzen filtration of $M_{\mathcal{O}}$ is the filtration

$$
M_{\mathcal{O}}=J_{0}\left(M_{\mathcal{O}}\right) \supseteq J_{1}\left(M_{\mathcal{O}}\right) \supseteq J_{2}\left(M_{\mathcal{O}}\right) \supseteq \cdots
$$

where $J_{k}\left(M_{\mathcal{O}}\right)=\left\{m \in M_{\mathcal{O}} \mid v_{x}(m) \geq k\right\}$.

Let $M_{F}=M_{\mathcal{O}} / x M_{\mathcal{O}}$. Then the Jantzen filtration of $M_{F}$ is the filtration

$$
M_{F}=J_{0}\left(M_{F}\right) \supseteq J_{1}\left(M_{F}\right) \supseteq J_{2}\left(M_{F}\right) \supseteq \cdots
$$

where $J_{k}\left(M_{F}\right)=\left(J_{k}\left(M_{\mathcal{O}}\right)+x M_{\mathcal{O}}\right) / x M_{\mathcal{O}} \cong J_{k}\left(M_{\mathcal{O}}\right) /\left(J_{k}\left(M_{\mathcal{O}}\right) \cap x M_{\mathcal{O}}\right)$, for $k \geq 0$. Since $M_{F}$ is finite dimensional, $J_{k}\left(M_{F}\right)=0$ for $k \gg 0$. The following easy observation of Jantzen's is the key to computing the Jantzen sum formula.

Lemma 4.1 (Jantzen) Suppose that $M_{\mathcal{O}}$ has a non-degenerate bilinear form. Then

$$
\sum_{k>0} \operatorname{dim} J_{k}\left(M_{F}\right)=v_{x}\left(G_{M_{\mathcal{O}}}\right)
$$

The proof is a straightforward calculation using the Smith normal form of $G_{M_{\mathcal{O}}}$, which exists because $\mathcal{O}$ is a principal ideal domain by assumption. For a proof that uses very similar language and notation to what we are using see [33, Lemma 5.30].

The cellular basis of Theorem 3.5 gives us a non-degenerate bilinear form on $S_{\mathcal{O}}^{\lambda}$, so we can apply Jantzen's constructions to the Specht modules $S_{\mathcal{O}}^{\lambda}$, for $\lambda \in \mathcal{P}_{n}^{\Lambda}$. Rather than looking at the Jantzen filtration directly we consider their direct sum.

Definition 4.2 Suppose that $\lambda \in \mathcal{P}_{n}^{\Lambda}$ and let $J_{F}^{\lambda}=\oplus_{k>0} J_{k}\left(S_{F}^{\lambda}\right)$. The Jantzen character of $S_{F}^{\lambda}$ is

$$
\operatorname{ch} J_{F}^{\lambda}=\sum_{k>0} \operatorname{ch} J_{k}\left(S_{F}^{\lambda}\right) .
$$

The main results of this paper follow from an explicit description of the Jantzen character $\operatorname{ch} J_{F}^{\lambda}$. The next result is our first step towards a useful description of this character.

Proposition 4.3 Let $\lambda \in \mathcal{P}_{n}^{\Lambda}$. Then $\operatorname{ch} J_{F}^{\lambda}=\sum_{\mathbf{i} \in I^{n}} v_{x}\left(\operatorname{det} G_{\mathbf{i}}^{\lambda}\right) \mathbf{i}$.

Proof As an $\mathcal{O}$-module, $S_{\mathcal{O}}^{\lambda}=\bigoplus_{\mathbf{i} \in I^{n}} S_{\mathcal{O}}^{\lambda} f_{\mathbf{i}}$. By construction, $\left\{b_{\mathfrak{s}} \mid \mathfrak{s} \in \operatorname{Std}_{\mathbf{i}}(\lambda)\right\}$ is a basis of $S_{\mathcal{O}}^{\lambda} f_{\mathbf{i}}$ and $G_{\mathbf{i}}^{\lambda}$ is the Gram matrix of the bilinear form on $S_{\mathcal{O}}^{\lambda}$ restricted to $S_{\mathcal{O}}^{\lambda} f_{\mathbf{i}}$. Moreover, in view of Lemma 3.21, if $\mathbf{i} \neq \mathbf{j} \in I^{n}$ then the summands $S_{\mathcal{O}}^{\lambda} f_{\mathbf{i}}$ and $S_{\mathcal{O}}^{\lambda} f_{\mathbf{j}}$ are orthogonal with respect to the inner product on $S_{\mathcal{O}}^{\lambda}$. Therefore, $J_{k}\left(S_{\mathcal{O}}^{\lambda}\right) f_{\mathbf{i}}=J_{k}\left(S_{\mathcal{O}}^{\lambda} f_{\mathbf{i}}\right)$ and $J_{k}\left(S_{F}^{\lambda}\right) f_{\mathbf{i}}=$ $J_{k}\left(S_{F}^{\lambda} f_{\mathbf{i}}\right)$, for all $\mathbf{i} \in I^{n}$. (As in Sect. 3.4, we are abusing notation and identifying $f_{\mathbf{i}} \in \mathscr{H}_{n}^{\Lambda}(\mathcal{O})$ and $f_{\mathbf{i}} \otimes 1_{F} \in \mathscr{H}_{n}^{\Lambda}$.) Hence,

$$
\begin{aligned}
\operatorname{ch} J_{F}^{\lambda} & =\sum_{k>0} \operatorname{ch} J_{k}\left(S_{F}^{\lambda}\right)=\sum_{\mathbf{i} \in I^{n}} \sum_{k>0} \operatorname{dim} J_{k}\left(S_{F}^{\lambda}\right) f_{\mathbf{i}} \cdot \mathbf{i}=\sum_{\mathbf{i} \in I^{n}} \sum_{k>0} \operatorname{dim} J_{k}\left(S_{F}^{\lambda} f_{\mathbf{i}}\right) \cdot \mathbf{i} \\
& =\sum_{\mathbf{i} \in I^{n}} v_{x}\left(\operatorname{det} G_{\mathbf{i}}^{\lambda}\right) \mathbf{i},
\end{aligned}
$$

where the last equality follows by applying Lemma 4.1 to $S_{\mathcal{O}}^{\lambda} f_{\mathbf{i}}$ and $S_{F}^{\lambda} f_{\mathbf{i}}$. Note that Lemma 4.1 applies because the bilinear form on $S_{\mathcal{K}}^{\lambda} f_{\mathbf{i}}$ is non-degenerate by Lemma 3.21. This completes the proof.

Lemma 3.21 gives an explicit formula for $\operatorname{det} G_{\mathbf{i}}^{\lambda}$ in terms of the $\gamma$-coefficients. The next step is to calculate $v_{x}\left(\gamma_{\mathfrak{t}}\right)$, for $\mathfrak{t} \in \operatorname{Std}(\lambda)$. First, a small interlude on cyclotomic polynomials. 


\subsection{Cyclotomic polynomials}

This section contains some brief reminders on cyclotomic polynomials that we need to the compute the Jantzen characters. All of the facts that we quote are standard and can be found, for example, in [28, VI §3].

Let $f \geq 1$. The $f$ th cyclotomic polynomial in $\mathbb{C}[x]$ is

$$
\Phi_{f}(x)=\prod_{\substack{1 \leq d \leq f \\ \operatorname{gcd}(d, f)=1}}\left(x-\exp \left(\frac{2 \pi i d}{f}\right)\right)
$$

Since $\Phi_{f}(x)$ is fixed by complex conjugation it follows that $\Phi_{f}(x) \in \mathbb{R}[x]$. In fact, $\Phi_{f}(x) \in$ $\mathbb{Z}[x]$. Hence, by base change, we consider $\Phi_{f}(x)$ as a polynomial in $F[x]$.

For this paper, a trivial but important observation is that $x^{f}-1=\prod_{d \mid f} \Phi_{d}(x)$. Therefore,

$$
[f]_{x}=\frac{x^{f}-1}{x-1}=\prod_{\substack{1<d \leq f \\ d \mid f}} \Phi_{d}(x) .
$$

In particular, if $p$ is a prime integer then $\Phi_{p}(x)=[p]_{x}=1+x+\cdots+x^{p-1}$. Moreover, if $f>1$ then

$$
\Phi_{f p}(x)= \begin{cases}\frac{\Phi_{f}\left(x^{p}\right)}{\Phi_{f}(x)}, & \text { if } p \nmid f, \\ \Phi_{f}\left(x^{p}\right), & \text { if } p \mid f .\end{cases}
$$

This recurrence relation uniquely determines $\Phi_{d}(x)$ as a polynomial in $F[x]$.

Recall that $z=x+\xi$, that $e>0$ is minimal such that $[e]_{\xi}=0$, and that $F$ is a field of characteristic $p \geq 0$. We can now state and prove the main fact that we need about cyclotomic polynomials.

Lemma 4.6 Suppose that $F$ is an algebraically closed field of characteristic $p \geq 0$ and that $f \geq 1$. Then

$$
v_{x}\left(\Phi_{f}(z)\right)= \begin{cases}1, & \text { if } f=e, \\ (p-1) p^{r-1}, & \text { if } p>0 \text { and } f=e p^{r} \text { for some } k>0, \\ 0, & \text { otherwise. }\end{cases}
$$

Proof First suppose that $p=0$. Since $F$ is algebraically closed, it contains a complete set $\left\{\omega_{1}, \ldots, \omega_{\phi_{f}}\right\}$ of primitive $f$ th roots of unity and $\Phi_{f}(x)=\prod_{k=1}^{\phi_{f}}\left(x-\omega_{k}\right)$. The constant term of $\Phi_{f}(z)$ is $\Phi_{f}(\xi)$, which is non-zero if and only if $e \neq f$ since $\xi$ is a primitive $e$ th root of unity. In particular, $v_{x}\left(\Phi_{f}(z)\right)=0$ if $f \neq e$. On the other hand, if $f=e$ then $\xi=\omega_{s}$, for some $s$, so the coefficient of $x$ in $\Phi_{e}(z)$ is $\prod_{k \neq s}\left(\xi-\omega_{k}\right) \neq 0$, so $v_{x}\left(\Phi_{e}(z)\right)=1$. Therefore, if $p=0$ then $v_{x}\left(\Phi_{f}(z)\right)=\delta_{e f}$.

Now suppose that $p>0$. Write $f=f^{\prime} p^{r}$, for integers $f^{\prime}$ and $r \geq 0$ such that $\operatorname{gcd}\left(f^{\prime}, p\right)=1$. If $r=0$, so that $f=f^{\prime}$, then the constant term of $\Phi_{f}(z)$ is $\Phi_{f}(\xi)$ which is non-zero if and only if $e=f=f^{\prime}$. Hence, by the argument of the last paragraph, $v_{x}\left(\Phi_{f}(z)\right)=\delta_{e f}$ when $r=0$. Finally, suppose that $r>0$. By (4.5) and the fact that we are in characteristic $p>0$,

$$
\Phi_{f}(z)=\left(\Phi_{f^{\prime}}(z)\right)^{(p-1) p^{r-1}}
$$


Therefore, $v_{x}\left(\Phi_{f}(z)\right)=(p-1) p^{r-1} v_{x}\left(\Phi_{f^{\prime}}(z)\right)=(p-1) p^{r-1} \delta_{e f^{\prime}}$, completing the proof.

\subsection{Computing Jantzen characters}

In [16, Theorem 3.13], Hu and the author gave a closed formula for $\operatorname{det} G^{\lambda}$ using the KLR $f$-degree functions (2.5) on standard tableaux

$$
\operatorname{deg}_{f}: \operatorname{Std}(\boldsymbol{\lambda}) \longrightarrow \mathbb{Z} ; \mathfrak{t} \mapsto \operatorname{deg}_{f}(\mathfrak{t}),
$$

for $f \geq 2$. We need to generalise this result to give a formula for $\operatorname{det} G_{\mathbf{i}}^{\lambda}$, for $\mathbf{i} \in I^{n}$.

First, recall the definition of the quantum integer $[k]_{z}$ from (3.1). For $\lambda \in \mathcal{P}_{n}^{\Lambda}$ define

$$
[\lambda]_{z}^{!}=\prod_{l=1}^{\ell} \prod_{r \geq 0}\left[\lambda_{r}^{(l)}\right]_{z}^{!}
$$

where for $k>0$ the quantum factorial of $k$ is $[k]_{z}^{!}=[k]_{z}[k-1]_{z} \ldots[1]_{z}$. For convenience, set $[0]_{z}^{!}=1$.

An analogue of the next result is implicit in the proof of [16, Theorem 3.13].

Lemma 4.7 Suppose that $\mathfrak{t} \in \operatorname{Std}(\lambda)$, for $\lambda \in \mathcal{P}_{n}^{\Lambda}$. Then there exists an integer $g_{\mathfrak{t}} \in \mathbb{N}$ such that

$$
\gamma_{\mathfrak{t}}=z^{g_{\mathfrak{t}}} \prod_{f>1} \Phi_{f}(x)^{\operatorname{deg}_{f}(\mathfrak{t})}
$$

Proof The proof is by induction on the dominance order on $\operatorname{Std}(\lambda)$. By [16, Proposition 3.11], which is a straightforward calculation using (3.8),

$$
\begin{aligned}
\gamma_{t^{\lambda}} & =[\lambda]_{z}^{!} \prod_{1 \leq l<m \leq \ell} \prod_{(l, r, c) \in[\lambda]}\left(\left[\kappa_{l}+c-r\right]_{z}-\left[\kappa_{m}\right]_{z}\right) \\
& =\prod_{(l, r, c) \in[\lambda]}[c]_{z} \prod_{l<m \leq \ell} z^{\kappa_{m}}\left[\kappa_{l}+c-r-\kappa_{m}\right]_{z},
\end{aligned}
$$

Let $A=(l, r, c) \in[\lambda]$ be a node in $\lambda$ and set $r=\mathfrak{t}^{\lambda}(A)$ and $\boldsymbol{\mu}=\operatorname{Shape}\left(\mathfrak{t}_{\downarrow}^{\lambda}\right)$. Recalling (4.4), and the definition of the integer $d_{A, f}(\boldsymbol{\mu})$ from (2.10), the contribution of the node $A$ to $\gamma_{t^{\lambda}}$ is

$$
\begin{gathered}
{[c]_{z} \prod_{l<m \leq \ell} z^{\kappa_{m}}\left[\kappa_{l}+c-r-\kappa_{m}\right]_{z}} \\
=z^{\kappa_{l+1}+\cdots+\kappa_{\ell}} \prod_{f>1} \Phi_{f}(z)^{d_{A, f}(\boldsymbol{\mu})} .
\end{gathered}
$$

Hence, the lemma holds when $\mathfrak{t}=\mathfrak{t}^{\lambda}$.

Now suppose that $\mathfrak{t} \neq \mathfrak{t}^{\lambda}$. Then there exists a tableau $\mathfrak{s} \in \operatorname{Std}(\lambda)$ such that $\mathfrak{s} \triangleright \mathfrak{t}=$ $\mathfrak{s}(r, r+1)$, where $1 \leq r<n$. By Corollary 3.12, and induction,

$$
\gamma_{\mathfrak{t}}=\frac{\left[1+\rho_{r}(\mathfrak{t})\right]_{z}\left[1-\rho_{r}(\mathfrak{t})\right]_{z}}{\left[\rho_{r}(\mathfrak{t})\right]_{z}^{2}} \gamma_{\mathfrak{s}}=\frac{\left[1+\rho_{r}(\mathfrak{t})\right]_{z}\left[1-\rho_{r}(\mathfrak{t})\right]_{z}}{\left[\rho_{r}(\mathfrak{t})\right]_{z}^{2}} z^{g_{\mathfrak{s}}} \prod_{f>1} \Phi_{f}(x)^{\operatorname{deg}_{f}(\mathfrak{s})} .
$$


Let $\mathbf{i}=r(\mathfrak{t})$. In the graded Specht module $\mathbb{S}^{\lambda}, \psi_{\mathfrak{t}}=\psi_{\mathfrak{s}} \psi_{r}$ with the degrees adding. Therefore,

$$
\operatorname{deg}_{f}(\mathfrak{t})=\operatorname{deg}_{f}(\mathfrak{s})-\left(\alpha_{i_{r}}, \alpha_{i_{r+1}}\right)=\operatorname{deg}_{f}(\mathfrak{s})+\left\{\begin{array}{l}
1, \quad \text { ifx } 1 \pm \rho_{r}(\mathfrak{t}) \equiv 0(\bmod f), \\
-2, \text { if } \rho_{r}(\mathfrak{t}) \equiv 0(\bmod f), \\
0, \quad \text { otherwise }
\end{array}\right.
$$

Hence, by induction, $\gamma_{t}$ can be written in the required form. This completes the proof.

We are one definition away from our first description of the Jantzen characters. First, recall that $e$ is the quantum characteristic of $\xi \in F$, so that $e$ is the minimal positive integer such that $[e]_{\xi}=0$. That is, either $\xi=1$ and $e=\operatorname{char} F$, or $\xi$ is a primitive $e$ th root of unity in $F$. Consequently, either $e=p$ or $\operatorname{gcd}(e, p)=1$.

Definition 4.9 Suppose that $\lambda \in \mathcal{P}_{n}^{\Lambda}$ and $\mathbf{i} \in I^{n}$. Define the $(e, \mathbf{i})$-degree of $\lambda$ to be the integer

$$
\operatorname{deg}_{e, \mathbf{i}}(\lambda)=\sum_{\mathfrak{t} \in \operatorname{Std}_{\mathbf{i}}(\lambda)} \operatorname{deg}_{e}(\mathfrak{t})
$$

Let $p$ be the characteristic of the field $F$. Define the $(e, p, \mathbf{i})$-degree of $\lambda$ to be

$$
\operatorname{deg}_{e, p, \mathbf{i}}(\boldsymbol{\lambda})=\operatorname{deg}_{e, \mathbf{i}}(\lambda)+\sum_{r \geq 1}(p-1) p^{r-1} \operatorname{deg}_{e p^{r}, \mathbf{i}}(\boldsymbol{\lambda})
$$

If $p=0$ then $\operatorname{deg}_{0}(\mathfrak{t})=0$ by (2.10), for all $\mathfrak{t} \in \operatorname{Std}\left(\mathcal{P}_{n}^{\Lambda}\right)$ Therefore, $\operatorname{deg}_{e, 0, \mathbf{i}}(\lambda)=$ $\operatorname{deg}_{e, \mathbf{i}}(\lambda)$.

The next result gives a complete description of the Jantzen characters. This formula has the advantage of being easy to compute in examples but it is not particularly useful in practice!

Proposition 4.10 Suppose that $\lambda \in \mathcal{P}_{n}^{\Lambda}$. Then $\operatorname{ch} J_{F}^{\lambda}=\sum_{\mathbf{i} \in I^{n}} \operatorname{deg}_{e, p, \mathbf{i}}(\lambda) \mathbf{i}$.

Proof By Theorem 3.5, $\mathscr{H}_{n}^{\Lambda}(F)$ is a cellular algebra so every field is splitting field for $\mathscr{H}_{n}^{\Lambda}(F)$ by Theorem 2.4. So, without loss of generality, we can assume that $F$ is algebraically closed. By Proposition 4.3 and Lemma 4.7,

$$
\begin{array}{rlr}
\operatorname{ch} J_{F}^{\lambda} & =\sum_{\mathbf{i} \in I^{n}} v_{x}\left(\operatorname{det} G_{\mathbf{i}}^{\lambda}\right) \mathbf{i} & \text { by Proposition 4.3, } \\
& =\sum_{\mathbf{i} \in I^{n}} v_{x}\left(\prod_{f>1} \Phi_{f}(z)^{\operatorname{deg}_{f, \mathbf{i}}(\lambda)}\right) \mathbf{i} & \text { by Lemma 4.7 } \\
& =\sum_{f>1}\left(\sum_{\mathbf{i} \in I^{n}} \operatorname{deg}_{f, \mathbf{i}}(\lambda) v_{x}\left(\Phi_{f}(z)\right)\right) \mathbf{i} & \\
& =\sum_{\mathbf{i} \in I^{n}} \operatorname{deg}_{e, p, \mathbf{i}}(\lambda) \mathbf{i},
\end{array}
$$

where the last equality follows by Lemma 4.6 and Definition 4.9. In particular, for the last equality observe that $v_{x}\left(\Phi_{f}(z)\right) \neq 0$ if and only if $f=e p^{r}$ for some $r \geq 0$ by Lemma 4.6.

As the coefficient of $\mathbf{i}$ in the Jantzen character is a non-negative integer we obtain the following combinatorial statement, refining results from [16, §3.3].

Corollary 4.11 Suppose that $\lambda \in \mathcal{P}_{n}^{\Lambda}$ and $\mathbf{i} \in I^{n}$. Then $\operatorname{deg}_{e, \mathbf{i}}(\lambda) \geq 0$ and $\operatorname{deg}_{e, p, \mathbf{i}}(\lambda) \geq 0$. 


\subsection{The positive Jantzen sum formula}

Building on Proposition 4.10, we are now ready to prove our main results, which describe the Jantzen characters as explicit linear combinations of the characters of $\mathscr{H}_{n}^{\Lambda}(F)$-modules.

The next definition extends Corollary 3.6.

Definition 4.12 Suppose that $F$ is a field. Set $E_{F}^{\mu}=\underline{\mathbb{E}}_{F}^{\mu}$, for $\mu \in \mathcal{K}_{n}^{\Lambda}$.

Using Theorem 3.4, we view $E_{F}^{\mu}$ as an $\mathscr{H}_{n}^{\Lambda}(F)$-module. The "traditional" way to define a module like $E_{F}^{\mu}$ is to construct a "decomposition map" by first choosing a modular system. We have sidestepped these additional complications by using the KLR algebra $\mathscr{R}_{n}^{\Lambda}(\mathbb{Z})$ to define $\mathbb{E}_{\mathbb{Z}}^{\mu}$ over $\mathbb{Z}$. In effect, we used the triple $(\mathbb{Q}, \mathbb{Z}, F)$ as a "modular system". We return to this theme in Proposition 4.18 below.

If $f(q) \in \mathcal{A}=\mathbb{Z}\left[q, q^{-1}\right]$ is a Laurent polynomial let $f^{\prime}(1)$ be the derivative of $f(q)$ evaluated at $q=1$. Define a linear map $\partial: \mathcal{A}\left[I^{n}\right] \longrightarrow \mathbb{Z}\left[I^{n}\right]$ by

$$
\partial\left(\sum_{\mathbf{i} \in I^{n}} f_{\mathbf{i}}(q) \mathbf{i}\right)=\sum_{\mathbf{i} \in I^{n}} f_{\mathbf{i}}^{\prime}(1) \mathbf{i} .
$$

By Remark 3.14, we can restrict $\partial$ to a linear map of Grothendieck groups $\partial: \operatorname{Rep} \mathscr{L}_{n} \longrightarrow$ $\operatorname{Rep} \mathscr{L}_{n}^{\prime}$.

For $\lambda \in \mathcal{P}_{n}^{\Lambda}$ and $\boldsymbol{\mu} \in \mathcal{K}_{n}^{\Lambda}$, recall from (2.15) that $d_{\lambda \mu}^{\mathbb{C}, e}(q)=\left[\mathbb{S}_{\mathbb{C}}^{\lambda}: \mathbb{D}_{\mathbb{C}}^{\mu}\right]_{q} \in \mathbb{N}\left[q, q^{-1}\right]$ is a characteristic zero graded decomposition number of $\mathscr{R}_{n}^{\Lambda}(\mathbb{C})$.

Lemma 4.13 Suppose that $f=e p^{r}$, for $r \geq 0$, and let $\lambda \in \mathcal{P}_{n}^{\Lambda}$ and $\boldsymbol{\mu} \in \mathcal{K}_{n}^{\Lambda_{f}}$. Then $\left(d_{\lambda \mu}^{\mathbb{C}, f}\right)^{\prime}(1) \geq 0$. Moreover, $\left(d_{\lambda \mu}^{\mathbb{C}, f}\right)^{\prime}(1) \neq 0$ only if $\lambda \triangleright \mu$.

Proof That $\left(d_{\lambda \mu}^{\mathbb{C}, f}\right)^{\prime}(1) \geq 0$ follows immediately from the deep fact that $d_{\lambda \mu}^{\mathbb{C}, e}(q) \in \delta_{\lambda \mu}+$ $q \mathbb{N}[q]$, which is proved in [5, Corollary 5.15]. The remaining claim that $\left(d_{\lambda \mu}^{\mathbb{C}, f}\right)^{\prime}(1) \neq 0$ only if $\lambda \triangleright \boldsymbol{\mu}$ also follows from the triangularity of the decomposition matrix with respect to dominance, which is a consequence of Theorem 3.5 and the general theory of cellular algebras.

According to the lemma, the coefficients in the character formula below are non-negative integers.

Theorem 4.14 Suppose that $F$ is a field of characteristic zero and let $\lambda \in \mathcal{P}_{n}^{\Lambda}$. Then

$$
\operatorname{ch} J_{F}^{\lambda}=\sum_{\mu \in \mathcal{K}_{n}^{\Lambda}}\left(d_{\lambda \mu}^{\mathbb{C}, e}\right)^{\prime}(1) \operatorname{ch} E_{F}^{\mu} .
$$

Proof Let $\mu \in \mathcal{K}_{n}^{\Lambda}$ and observe that $\partial \operatorname{ch}_{q} \mathbb{E}_{F}^{\mu}=0$ because $\overline{\operatorname{ch}_{q} \mathbb{E}_{F}^{\mu}}=\operatorname{ch}_{q} \mathbb{E}_{F}^{\mu}$ by Corollary 2.19. Therefore,

$$
\partial\left(d_{\lambda \mu}^{\mathbb{C}, e}(q) \operatorname{ch}_{q} \mathbb{E}_{F}^{\mu}\right)=\left(d_{\lambda \mu}^{\mathbb{C}, e}\right)^{\prime}(1) \operatorname{ch} E_{F}^{\mu}
$$

by the chain rule. Next, notice that Corollary 2.18 implies that

$$
\sum_{\mathbf{i} \in I^{n}} \sum_{\mathfrak{t} \in \operatorname{Std}_{\mathbf{i}}(\lambda)} q^{\operatorname{deg}_{e}(\mathfrak{t})} \mathbf{i}=\operatorname{ch}_{q} \mathbb{S}_{F}^{\lambda}=\sum_{\mu \in \mathcal{K}_{n}^{\Lambda}} d_{\lambda \mu}^{\mathbb{C}, e}(q) \operatorname{ch}_{q} \mathbb{E}_{F}^{\mu}
$$


Applying $\partial$ to both sides of this equation, using the previous remark, shows that

$$
\sum_{\mathbf{i} \in I^{n}} \sum_{\mathfrak{t} \in \operatorname{Std}_{\mathbf{i}}(\lambda)} \operatorname{deg}_{e}(\mathfrak{t}) \mathbf{i}=\sum_{\mu \in \mathcal{K}_{n}^{\Lambda}} d_{\lambda \mu}^{\prime}(1) \operatorname{ch} E_{F}^{\mu} .
$$

Since $\operatorname{deg}_{e, 0, \mathbf{i}}(\lambda)=\operatorname{deg}_{e, \mathbf{i}}(\mathbf{i})=\sum_{\mathfrak{t} \in \operatorname{Std}_{\mathbf{i}}(\lambda)} \operatorname{deg}_{e}(\mathfrak{t})$, for $\mathbf{i} \in I^{n}$, comparing the left hand side of the last displayed equation with Proposition 4.3 completes the proof.

The characters of the graded Specht modules are crucial to the proof of Theorem 4.14, however, the proof is purely combinatorial and it does not use a representation theoretic connection between the Jantzen filtration of $S_{F}^{\lambda}$ and the graded $\mathscr{R}_{n}^{\Lambda}(F)$-modules $\mathbb{E}_{F}^{\mu}$. It would be interesting to find a direct connection between the modules $J_{k}\left(S_{F}^{\lambda}\right)$ and $\mathbb{E}_{F}^{\mu}$.

The Jantzen sum formula is usually stated in the Grothendieck group Rep $\mathscr{H}_{n}^{\Lambda}$. In view of Proposition 3.16, Theorem 4.14 is equivalent to the following more "traditional" statement:

Corollary 4.15 Suppose that $p=0$ and let $\lambda \in \mathcal{P}_{n}^{\Lambda}$. Then

$$
\left[J_{F}^{\lambda}\right]=\sum_{k>1}\left[J_{k}\left(S_{F}^{\lambda}\right)\right]=\sum_{\mu \in \mathcal{K}_{n}^{\Lambda}}\left(d_{\lambda \mu}^{\mathbb{C}, e}\right)^{\prime}(1)\left[E_{F}^{\mu}\right] .
$$

Remark 4.16 In characteristic zero, Ryom-Hansen [39, Theorem 1] proved an analogue of this result for $\Lambda=\Lambda_{0}$ by assuming a conjecture of Rouquier's [29, §9] that, in the post KLR world, says that the Jantzen filtration of $\mathbb{S}_{\mathbb{C}}^{\lambda}$ coincides with its grading filtration. Yvonne [46, Theorem 2.11] extended Ryom-Hanson's work to arbitrary $\Lambda \in P_{e}^{+}$. Theorem 4.14 shows that the description of the Jantzen sum formula in terms of the integers $\left(d_{\lambda \mu}^{\mathbb{C}, e}\right)^{\prime}(1)$ is independent of Rouquier's conjecture.

We next compute the Jantzen characters for fields of positive characteristic $p>0$. As Proposition 4.10 and Definition 4.9 suggest, this involves looking at Hecke algebras at $e p^{r}$ th roots of unity for $r \geq 0$. We first need to set up the combinatorial and representation theoretic machinery to do this. When $r=0$ the tool that we need is provided by Theorem 2.16 but when $r>0$ we need to work harder.

If $f=e p^{r}$, for $r \geq 0$, let $\zeta_{f}=\exp \left(\frac{2 \pi \sqrt{-1}}{f}\right) \in \mathbb{C}$. Then $\zeta_{f}$ is a primitive $f$ th root of unity in $\mathbb{C}$. Let $I_{f}=\mathbb{Z} / f \mathbb{Z}$. (In particular, $I=I_{e}$.) Since $e$ divides $f$, there is a well-defined surjective map $I_{f} \longrightarrow I=I_{e} ; i \mapsto i_{f}$ given by "reducing modulo $e$ ". More explicitly, this map sends $a+f \mathbb{Z}$ to $a+e \mathbb{Z}$, for $a \in \mathbb{Z}$. Extending this notation, if $\mathbf{i} \in I_{f}^{n}$ let $\mathbf{i}_{f}$ be the corresponding sequence in $I^{n}=I_{e}^{n}$. This map induces an abelian group homomorphism

$$
\mathrm{L}_{f, e}: \mathbb{Z}\left[I_{f}^{n}\right] \longrightarrow \mathbb{Z}\left[I^{n}\right] ; \sum_{\mathbf{i} \in I_{f}^{n}} c_{\mathbf{i}} \mathbf{i} \mapsto \sum_{\mathbf{i} \in I_{f}^{n}} c_{\mathbf{i}} \mathbf{i}_{f}, \quad \text { for } c_{\mathbf{i}} \in \mathbb{Z}
$$

Recall from Sect. 2.3 that $P_{f}^{+}$and $Q_{f}^{+}$are the positive weight and positive root lattices attached to the quiver $\Gamma_{f}$, for $f \geq 2$. Abusing notation, let $\Lambda_{i}$ and $\alpha_{i}$ be fundamental weights and simple roots in the corresponding weight lattices for any $f \geq 2$, with the meaning being clear from the choice of index set $I_{f}$. In Sect. 2.3 we fixed a dominant weight $\Lambda=\Lambda_{e} \in P^{+}=P_{e}^{+}$. For $f=e p^{r}$ fix a dominant weight $\Lambda_{f} \in P_{f}^{+}$such that

$$
\left(\Lambda, \alpha_{i}\right)=\sum_{\substack{j \in I_{f} \\ j_{f}=i}}\left(\Lambda_{f}, \alpha_{j}\right), \quad \text { for all } i \in I .
$$

These conditions determine the dominant weight $\Lambda_{f}$ uniquely if and only if $r=0$. If $r>0$ then the results below are independent of the choice of $\Lambda_{f}$. 
Let $\mathscr{H}_{n}^{\Lambda_{f}}(\mathbb{C})$ be the cyclotomic Hecke algebra over $\mathbb{C}$ with Hecke parameter $\zeta_{f}$ and dominant weight $\Lambda_{f}$. For $\lambda \in \mathcal{P}_{n}^{\Lambda}$ let $\mathbb{S}_{\mathbb{C}, f}^{\lambda}$ be the graded Specht module for $\mathscr{R}_{n}^{\Lambda_{f}}(\mathbb{C})$ indexed by $\lambda$. Similarly, given an $\ell$-partition $\mu \in \mathcal{K}_{n}^{\Lambda_{f}}=\mathcal{K}_{f, n}^{\Lambda_{f}}$ let $\mathbb{E}_{\mathbb{C}, f}^{\mu}=\mathbb{D}_{\mathbb{C}, f}^{\mu}$ be the (almost) simple $\mathscr{H}_{n}^{\Lambda_{f}}(\mathbb{C})$-module defined by Definition 2.14 and let $S_{\mathbb{C}, f}^{\lambda}$ and $D_{\mathbb{C}, f}^{\lambda}$ be the corresponding Specht and simple modules for $\mathscr{H}_{n}^{\Lambda_{f}}(\mathbb{C})$. By (2.15), we have polynomials

$$
d_{\lambda \mu}^{\mathbb{C}, f}(q)=\left[\mathbb{S}_{\mathbb{C}, f}^{\lambda}: \mathbb{E}_{\mathbb{C}, f}^{\mu}\right]_{q}, \quad \text { for } \lambda \in \mathcal{P}_{n}^{\Lambda} \text { and } \boldsymbol{\mu} \in \mathcal{K}_{n}^{\Lambda_{f}} .
$$

Armed with this notation we can state the result that we need. This result is partly motivated by the discussion after [33, Conjecture 6.37].

Proposition 4.18 Suppose that $f=e p^{r}$, where $r \geq 0$, and let $K$ be a field of characteristic zero that contains $\zeta_{f}$. Then there is a unique homomorphism $A_{f, e}: \operatorname{Rep} \mathscr{H}_{n}^{\Lambda_{f}}(K) \longrightarrow$ $\operatorname{Rep} \mathscr{H}_{n}^{\Lambda}(F)$ such that the following diagram commutes:

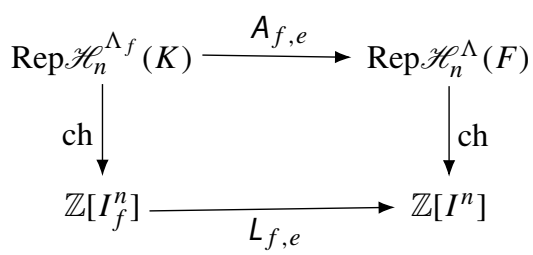

Moreover, $A_{f, e}\left[S_{K, f}^{\lambda}\right]=\left[S_{F}^{\lambda}\right]$, for all $\lambda \in \mathcal{P}_{n}^{\Lambda}$.

Proof The two character maps are injective by Proposition 3.16, so once we show that $A_{f, e}$ exists it is automatically unique. The cyclotomic Hecke algebras are cellular, so every field is a splitting field by Theorem 2.4. Hence, without loss of generality, we can assume that $K=\mathbb{Q}\left[\zeta_{f}\right]$ and $F=\mathbb{F}_{p}[\xi]$. Let $\mathcal{Z}_{f}=\mathbb{Z}\left[\zeta_{f}\right]$. Then $\mathscr{H}_{n}^{\Lambda_{f}}\left(\mathcal{Z}_{f}\right)$ is a $\mathcal{Z}_{f}$-subalgebra of $\mathscr{H}_{n}^{\Lambda_{f}}(K)$ and $\mathscr{H}_{n}^{\Lambda_{f}}(K) \cong \mathscr{H}_{n}^{\Lambda_{f}}\left(\mathcal{Z}_{f}\right) \otimes_{\mathcal{Z}_{f}} K$. Since $e$ divides $f$ there is a unique surjective ring homomorphism $\pi_{f}: \mathcal{Z}_{f} \rightarrow F$ such that $\pi_{f}\left(\zeta_{f}\right)=\xi$. Hence, we can consider $F$ as a $\mathcal{Z}_{f}$-algebra. We claim that, as $F$-algebras,

$$
\mathscr{H}_{n}^{\Lambda}(F) \cong \mathscr{H}_{n}^{\Lambda_{f}}\left(\mathcal{Z}_{f}\right) \otimes_{\mathcal{Z}_{f}} F .
$$

Since the cyclotomic Hecke algebras are free over any ring by Theorem 3.5, to prove this it is enough to check that tensoring with $\mathbb{F}_{p}[\xi]$ respects the relations. The only relation that is not obviously preserved is the cyclotomic relation, which can be written in the form $\prod_{i \in I_{f}}\left(L_{1}-[i]_{\zeta_{f}}\right)^{\left(\Lambda_{f}, \alpha_{i}\right)}=0$ by (3.3). This relation is preserved by tensoring with $\mathcal{Z}_{f}$ by (4.17) because $\left[i_{f}\right]_{\xi}=\pi_{f}\left([i]_{\zeta_{f}}\right)$, for all $i \in I_{f}$.

Set $\mathfrak{p}_{f}=\operatorname{ker} \pi_{f}$, a prime ideal in $\mathcal{Z}_{f}$, and let $\mathcal{O}_{f}=\left(\mathcal{Z}_{f}\right)_{\mathfrak{p}_{f}}$ be the localisation of $\mathcal{Z}_{f}$ at $\mathfrak{p}_{f}$. Then $\mathcal{O}_{f}$ is a discrete valuation ring with maximal ideal $\mathfrak{p} \mathcal{O}_{f}$, quotient field $K$ and residue field $F \cong \mathcal{O}_{f} / \mathfrak{p} \mathcal{O}_{f}$. Hence, the triple $\left(K, \mathcal{O}_{f}, F\right)$ is a $p$-modular system in the sense of $[8, \S 16 \mathrm{~A}]$. Therefore, there is a well-defined decomposition map $\mathrm{A}_{f, e}: \operatorname{Rep} \mathscr{H}_{n}^{\Lambda_{f}}(K) \longrightarrow$ $\operatorname{Rep} \mathscr{H}_{n}^{\Lambda}(F)$ that is independent of the choice of $\mathcal{O}_{f}$ by [8, Proposition 16.17]. To describe $\mathrm{A}_{f, e}$ explicitly, if $V$ is an $\mathscr{H}_{n}^{\Lambda_{f}}(K)$-module then $\mathrm{A}_{f, e}(V)$ is defined by choosing a full $\mathcal{O}_{f}$-lattice $V_{\mathcal{O}_{f}}$ in $V$ and then setting $A_{f, e}(V)=V_{\mathcal{O}_{f}} \otimes_{\mathcal{O}_{f}} F$. Consequently, because the 
cellular basis $\left\{b_{\mathfrak{s t}}\right\}$ of $\mathscr{H}_{n}^{\Lambda_{f}}(K)$ from Proposition 3.19 is defined over any ring, if $\lambda \in \mathcal{P}_{n}^{\Lambda}$ then $\mathrm{A}_{f, e}\left(S_{K}^{\lambda}\right)=S_{F}^{\lambda}$.

It remains to show that the diagram in the proposition commutes. Since $\mathrm{L}_{f, e}\left(\operatorname{ch} S_{K}^{\lambda}\right)=$ $\operatorname{ch}\left(S_{F}^{\lambda}\right)$, we have commutativity on the Specht modules. The decomposition matrix $\mathrm{D}_{K, f}$ is unitriangular, so $\left\{S_{F}^{\lambda} \mid \lambda \in \mathcal{K}_{n}^{\Lambda_{f}}\right\}$ is a basis for $\operatorname{Rep} \mathscr{H}_{n}^{\Lambda_{f}}(K)$. Hence, this completes the proof.

Definition 4.19 Suppose that $f=e p^{r}$, for $r \geq 0$, and let $\boldsymbol{v} \in \mathcal{K}_{n}^{\Lambda_{f}}$. Set $E_{f, e}^{\boldsymbol{v}}=\mathrm{A}_{f, e}\left(E_{\mathbb{C}, f}^{\boldsymbol{v}}\right)$.

Strictly speaking, $E_{f, e}^{v}$ is the image of a module in the Grothendieck group of $\mathscr{H}_{n}^{\Lambda}(F)$ but we abuse notation refer to it as the modular reduction of the almost simple module $E_{\mathbb{C}}^{\mu}$ for $\mathscr{H}_{n}^{\Lambda_{f}}(\mathbb{C})$. (By the proof of Proposition 4.18, the module $E_{f, e}^{v}$ is well-defined only up to a choice of lattice whereas $\left[E_{f, e}^{v}\right]$ is independent of this choice.) In the special case when $e=f$ or, equivalently, $r=0$, comparing characters and applying Proposition 4.18 and Definition 2.14 yields the following:

Corollary 4.20 Let $\lambda \in \mathcal{P}_{n}^{\Lambda}$ and $\boldsymbol{\mu} \in \mathcal{K}_{n}^{\Lambda}$. Then $S_{F}^{\lambda}=A_{e, e}\left(S_{\mathbb{C}, e}^{\lambda}\right)$ and $E_{F}^{\mu}=A_{e, e}\left(E_{\mathbb{C}, e}^{\mu}\right)$.

This gives the following generalisation of the (ungraded analogue of) Theorem 2.16. Define the $(f, e)$-adjustment matrix $\mathrm{A}_{f, e}=\left(a_{\boldsymbol{v} \mu}^{f, e}\right)$, for $\boldsymbol{v} \in \mathcal{K}_{n}^{\Lambda_{f}}$ and $\boldsymbol{\mu} \in \mathcal{K}_{n}^{\Lambda}$, by

$$
a_{\boldsymbol{v} \mu}^{f, e}=\left[E_{f, e}^{v}: D_{F}^{\mu}\right] .
$$

The next result is a generalisation of the ungraded, or $q=1$, analogue of (2.17).

Corollary 4.21 Suppose that $f=e p^{r}$, for $r \geq 0$. Then $D_{F, e}=D_{\mathbb{C}, f} A_{f, e}$.

Proof If $\lambda \in \mathcal{P}_{n}^{\Lambda}$ then $\left[S_{\mathbb{C}, f}^{\lambda}\right]=\sum_{\nu} d_{\lambda \nu}^{\mathbb{C}, f}\left[E_{\mathbb{C}, f}^{v}\right]$ in $\operatorname{Rep} \mathscr{H}_{n}^{\Lambda_{f}}(\mathbb{C})$, where the sum is over $\boldsymbol{v} \in \mathcal{K}_{n}^{\Lambda_{f}}$. Applying the linear map $\mathrm{A}_{f, e}$ from Proposition 4.18 for the second equality,

$$
\left[S_{F}^{\lambda}\right]=\sum_{\boldsymbol{v} \in \mathcal{K}_{n}^{\Lambda_{f}}} d_{\lambda \boldsymbol{v}}^{\mathbb{C}, f}\left[E_{f, e}^{\boldsymbol{v}}\right]=\sum_{\boldsymbol{v} \in \mathcal{K}_{n}^{\Lambda_{f}}} d_{\lambda \boldsymbol{v}}^{\mathbb{C}, f}\left(\sum_{\boldsymbol{\mu} \in \mathcal{K}_{n}^{\Lambda}} a_{\boldsymbol{v} \mu}^{f, e}\left[D_{F}^{\mu}\right]\right)=\sum_{\boldsymbol{\mu} \in \mathcal{K}_{n}^{\Lambda}}\left(\sum_{\boldsymbol{v} \in \mathcal{K}_{n}^{\Lambda_{f}}} d_{\lambda \boldsymbol{v}}^{\mathbb{C}, f} a_{\boldsymbol{v} \mu}^{f, e}\right)\left[D_{F}^{\mu}\right] .
$$

Since $\left[S_{F}^{\lambda}\right]=\sum_{\mu} d_{\lambda \mu}^{F, e}\left[D_{F}^{\mu}\right]$ the result follows.

In the special case when $e=f$, Corollary 4.20 implies that $\mathrm{A}_{e, e}=\mathrm{A}_{F, e}(1)$, where $\mathrm{A}_{F, e}(1)$ is the adjustment matrix obtained by setting $q=1$ in the graded adjustment matrix $\mathrm{A}_{F, e}(q)$ of (2.17).

Remark 4.22 By Theorem 2.16, Corollary 4.21 extends to give graded adjustment matrices in the special case when $e=f$ or, equivalently, $r=0$. If $r>0$ then $\mathscr{R}_{n}^{\Lambda_{f}}(\mathbb{C}) \cong \mathscr{H}_{n}^{\Lambda_{f}}(\mathbb{C})$ by Theorem 3.4. By Definition 2.5 we have a cyclotomic KLR algebra $\mathscr{R}_{n}^{\Lambda_{f}}(F)$ defined over $F$, however, Definition 3.2 does not give a definition of a cyclotomic Hecke algebra $\mathscr{H}_{n}^{\Lambda_{f}}(F)$ because a field of characteristic $p>0$ cannot contain a primitive $f$ th root of unity when $p$ divides $f$. Consequently, Theorem 3.4 does not apply to $\mathscr{R}_{n}^{\Lambda_{f}}(F)$ and, a priori, the algebra $\mathscr{R}_{n}^{\Lambda_{f}}(F)$ is not isomorphic to a cyclotomic Hecke algebra. Even so, Theorem 2.11 shows that $\mathscr{R}_{n}^{\Lambda_{f}}(F)$ is a graded cellular algebra that comes equipped with Specht modules $\mathbb{S}_{F}^{\lambda}$, simple modules $\mathbb{D}_{F}^{\mu}$ and almost simple modules $\mathbb{E}_{F}^{\mu}$, for $\lambda \in \mathcal{P}_{n}^{\Lambda}$ and $\mu \in \mathcal{K}_{n}^{\Lambda_{f}}$. Let $K$ be the field 
used in the proof of Proposition 4.18. Then $\mathscr{H}_{n}^{\Lambda_{f}}(K) \cong \mathscr{R}_{n}^{\Lambda_{f}}(K)$ and reduction modulo $p$ induces a decomposition map from $\operatorname{Rep} \mathscr{R}_{n}^{\Lambda_{f}}(K)$ to $\operatorname{Rep} \mathscr{R}_{n}^{\Lambda_{f}}(F)$, so the decomposition map of Proposition 4.18 factors through $\operatorname{Rep} \mathscr{R}_{n}^{\Lambda_{f}}(F)$, however, it is not clear how to define a map between the Grothendieck groups of $\operatorname{Rep} \mathscr{R}_{n}^{\Lambda_{f}}(F)$ and $\operatorname{Rep} \mathscr{R}_{n}^{\Lambda}(F)$.

Using formal characters, it is straightforward to compute the following graded decomposition matrices corresponding to the choices $e=p=2, \Lambda=\Lambda_{0}, f=4$ and $\Lambda_{f}=\Lambda_{0}$ (so $F=\mathbb{F}_{2}$ is the field of order 2):

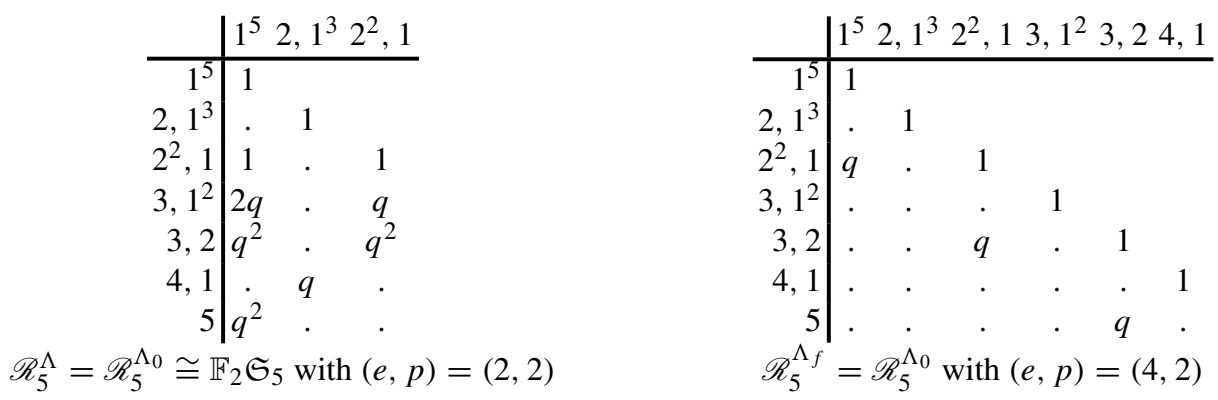

Comparing these two matrices shows that there is no graded adjustment matrix with entries in $\mathbb{N}\left[q, q^{-1}\right]$ in this case. (In contrast, in agreement with Proposition 4.18, there is an adjustment matrix when we set $q=1$, which corresponds to forgetting the grading.) Hence, when $r>0$ we cannot expect the naive generalisations of Proposition 4.18 and Corollary 4.21 to hold in the graded setting. It would be interesting to find a direct connection between $\mathscr{R}_{n}^{\Lambda_{f}}(F)$ and $\mathscr{R}_{n}^{\Lambda}(F)$ as graded algebras.

We are now able to prove what is really the main result of this paper.

Theorem 4.23 Suppose that $F$ is a field of characteristic $p>0$ and let $\lambda \in \mathcal{P}_{n}^{\Lambda}$. Then

$$
\operatorname{ch} J_{F}^{\lambda}=\sum_{\mu \in \mathcal{K}_{n}^{\Lambda}}\left(d_{\lambda \mu}^{\mathbb{C}, e}\right)^{\prime}(1) \operatorname{ch} E_{F}^{\mu}+\sum_{f=e p^{r}>e}(p-1) p^{r-1} \sum_{\boldsymbol{v} \in \mathcal{K}_{n}^{\Lambda_{f}}}\left(d_{\lambda \nu}^{\mathbb{C}, f}\right)^{\prime}(1) \operatorname{ch} E_{f, e}^{v} .
$$

Moreover, all of the coefficients on the right hand side are non-negative integers.

Proof Arguing as in the proof of Theorem 4.14, if $f=e p^{r}$ for $r \geq 0$ then

$$
\sum_{\mathbf{i} \in I_{f}^{n}} \operatorname{deg}_{f}(\lambda) \mathbf{i}=\sum_{\boldsymbol{v} \in \mathcal{K}_{n}^{\Lambda_{f}}}\left(d_{\lambda \boldsymbol{v}}^{\mathbb{C}, f}\right)^{\prime}(1) \operatorname{ch} E_{\mathbb{C}}^{\boldsymbol{v}}
$$

Applying the $\operatorname{mapL}_{f, e}$, and using Proposition4.18 and Definition 4.19, this becomes

$$
\sum_{\mathbf{i} \in I_{f}^{n}} \operatorname{deg}_{f}(\lambda) \mathbf{i}_{f}=\sum_{\boldsymbol{v} \in \mathcal{K}_{n}^{\Lambda_{f}}}\left(d_{\lambda \boldsymbol{v}}^{\mathbb{C}, f}\right)^{\prime}(1) \operatorname{ch} E_{f, e}^{\boldsymbol{v}} .
$$

Multiplying by $(p-1) p^{r-1}$ if $r>0$ and then summing over $r$, as in Definition4.9,

$$
\sum_{\mathbf{i} \in I^{n}} \operatorname{deg}_{e, p, \mathbf{i}}(\lambda) \mathbf{i}=\sum_{\boldsymbol{\mu} \in \mathcal{K}_{n}^{\Lambda}} d_{\lambda \mu}^{\prime}(1) \operatorname{ch} E_{F}^{\mu}+\sum_{r>0}(p-1) p^{r-1} \sum_{\boldsymbol{v} \in \mathcal{K}_{n}^{\Lambda_{f}}}\left(d_{\lambda \boldsymbol{v}}^{\mathbb{C}, f}\right)^{\prime}(1) \operatorname{ch} E_{f, e}^{\boldsymbol{v}} .
$$

By Lemma 4.13, $\left(d_{\lambda v}^{\mathbb{C}, f}\right)^{\prime}(1)=0$ unless $\lambda \triangleright \boldsymbol{v}$. Notice that $f>e$ if and only if $r>0$ since we are assuming that $p>0$ in this result. (In contrast, these two conditions are not quite equivalent in the statement of our Main Theorem because the latter result allows $p=0$.) 
Hence, the character formula for ch $J_{F}^{\lambda}$ follows by Proposition 4.10. Finally, the coefficients on the right hand side of the character formula for $\mathrm{ch} J_{F}^{\lambda}$ are non-negative integers by Lemma 4.13 .

Applying Proposition 3.16, just as we did after Theorem 4.14, we obtain the Main Theorem from the introduction:

Corollary 4.24 Suppose that $F$ is a field of characteristic $p>0$ and let $\lambda \in \mathcal{P}_{n}^{\Lambda}$. Then

$$
\sum_{k>0}\left[J_{k}\left(S_{F}^{\lambda}\right)\right]=\sum_{\boldsymbol{\mu} \in \mathcal{K}_{n}^{\Lambda}}\left(d_{\lambda \mu}^{\mathbb{C}, e}\right)^{\prime}(1)\left[E_{F}^{\mu}\right]+\sum_{f=e p^{r}>e}(p-1) p^{r-1} \sum_{\boldsymbol{v} \in \mathcal{K}_{n}^{\Lambda_{f}}}\left(d_{\lambda \boldsymbol{v}}^{\mathbb{C}, f}\right)^{\prime}(1)\left[E_{f, e}^{\boldsymbol{v}}\right] .
$$

One of the applications of the classical Jantzen sum formula is to determine when $S_{F}^{\lambda}=$ $D_{F}^{\lambda}$, for $\lambda \in \mathcal{P}_{n}^{\Lambda}$. If $F$ is a field of characteristic zero then Theorem 4.14 implies that $S_{F}^{\lambda}$ is irreducible if and only if $d_{\lambda \mu}^{\mathbb{C}, e}=0$ for $\mu \neq \lambda$, which is true but not very insightful. If $p>0$ we obtain new information.

Corollary 4.25 Suppose that $F$ is a field of characteristic $p>0$ and let $\lambda \in \mathcal{P}_{n}^{\Lambda}$. Then $S_{F}^{\lambda}=D_{F}^{\lambda}$ if and only $S_{\mathbb{C}, f}^{\lambda}=D_{\mathbb{C}, f}^{\lambda}$, for all $f=e p^{r}$ and $r \geq 0$.

Although this result does not appear to be the literature it can be deduced from [19, Theorem 4.7(iii)].

In a similar vein, de Boeck et al [9, Theorem 1.2] have shown that in positive characteristic the dimensions of the simple modules indexed by two column partitions for the Hecke algebra of the symmetric with $\xi$ of quantum characteristic $e$ depend upon the dimensions of the simple modules at $e p^{r}$ th roots of unity in characteristic zero. It would be interesting to find stronger connections between the representation theories of the Hecke algebras with quantum characteristic $e$ in characteristic $p>0$ and the Hecke algebras in characteristic zero at $e p^{r}$ th roots of unit, for $r \geq 0$.

A second application of the Jantzen sum formula is to give upper bound for decomposition numbers. For $\lambda \in \mathcal{P}_{n}^{\Lambda}$ and $\boldsymbol{\mu} \in \mathcal{K}_{n}^{\Lambda}$ define

$$
j_{\lambda \mu}^{F, e}=\left(d_{\lambda \mu}^{\mathbb{C}, e}\right)^{\prime}(1) a_{\lambda \mu}+\sum_{r>0}(p-1) p^{r-1} \sum_{\substack{f=e p^{r} \\ \boldsymbol{v} \in \mathcal{K}_{n}^{\Lambda_{f}}}}\left(d_{\lambda \boldsymbol{v}}^{\mathbb{C}, f}\right)^{\prime}(1) a_{\boldsymbol{v} \mu}^{f, e} .
$$

Then comparing the coefficient of $\operatorname{ch} D_{F}^{\mu}$ on both sides of Theorem 4.23 shows that the following holds.

Corollary 4.26 Suppose that $\lambda \in \mathcal{P}_{n}^{\Lambda}$ and $\boldsymbol{\mu} \in \mathcal{K}_{n}^{\Lambda}$, with $\boldsymbol{\mu} \neq \lambda$. Then $d_{\lambda \mu}^{F, e} \leq j_{\lambda \mu}^{F, e}$.

In particular, $d_{\lambda \mu}^{F, e} \neq 0$ only if $j_{\lambda \mu}^{F, e} \neq 0$.

We find it striking that the upper bound on the decomposition numbers of $\mathscr{H}_{n}^{\Lambda}(F)$ increases dramatically as soon as $d_{\lambda v}^{\mathbb{C}, f}(q)$ is non-zero, for some $f=e p^{r}$. In the special case when $\Lambda=\Lambda_{0}$, so that $\mathscr{H}_{n}^{\Lambda}(F)$ is an Iwahori-Hecke algebra of the symmetric group, Theorem 4.23 provides some evidence in favour of (the now disproved) James' conjecture $[17, \S 4]$. Both the statement and proof use the standard terminology of the weight, hooks and cores of partitions; see, for example, [33, Chapter 4].

Corollary 4.27 Suppose that $\Lambda=\Lambda_{0}$ and that $\lambda \in \mathcal{P}_{n}^{\Lambda}$ is partition e-weight $w<p$. Then

$$
\operatorname{ch} J_{F}^{\lambda}=\operatorname{ch} J_{\mathbb{C}}^{\lambda}=\sum_{\mu \in \mathcal{K}_{n}^{\Lambda}}\left(d_{\lambda \mu}^{\mathbb{C}, e}\right)^{\prime}(1) \operatorname{ch} E_{F}^{\mu} .
$$


Proof Let $f=e p^{r}$, where $r>0$. Since $w<p$, the partition $\lambda$ cannot contain a removable $f$ hook. Therefore, $\lambda$ is an $f$-core and the Specht module $S_{\mathbb{C}, f}^{\lambda}=E_{\mathbb{C}, f}^{\lambda}=D_{\mathbb{C}, f}^{\lambda}$ is irreducible. Hence, $d_{\lambda \mu}^{\mathbb{C}, f}(q)=\delta_{\lambda \mu}$ and Theorem 4.23 implies the result.

In general we would not expect the character ch $J_{F}^{\lambda}$ to determine the Jantzen filtration uniquely. Combining Corollary 4.27 with Williamson's counterexample to the James conjecture $[44, \S 8]$, shows that $\operatorname{ch} J_{F}^{\lambda}$ does not determine the Jantzen filtration uniquely even when $w<p$.

\section{The classical sum formula}

For completeness, this section shows how to use formal characters to prove a more "classical" version of the Jantzen sum formula [18,19]. In the cyclotomic case, the sum formula that we obtain is equivalent to, but slightly nicer than, that given by [19, Theorem 4.3]. Mostly this is because Definition 3.2 is implicitly invoking the Morita equivalence of [11, Theorem 1.1] to restrict the "cyclotomic parameters" of $\mathscr{H}_{n}^{\Lambda}(F)$ to a single $\xi$-orbit. Two other reasons why the results in this section are more elegant than the corresponding result [19] are because we have already fixed the quantum characteristic $e$ and because the combinatorial framework that we introduce directly links beta numbers and contents for $\ell$-partitions.

As in Sect. 3.3, throughout this section we fix a field $F$ and a primitive $e$ th root of unity $\xi \in F$, where $e<\infty$, and study the Hecke algebra $\mathscr{H}_{n}^{\Lambda}(F)$. We continue to assume that the charge $\kappa$ satisfies (3.7) and we consider the algebras $\mathscr{H}_{n}^{\Lambda}(\mathcal{O})$ and $\mathscr{H}_{n}^{\Lambda}(\mathcal{K})$ with Hecke parameter $z=x+\xi$, where $\mathcal{O}=F[x]_{(x)}$ and $\mathcal{K}=F(x)$.

\subsection{Charged beta numbers}

We want an analogue of the branching rules for the Gram determinants $G_{\mathbf{i}}^{\lambda}$. This requires a mild dose of new notation that is motivated by $[19, \S 3]$.

The length of a partition $\lambda=\left(\lambda_{1} \geq \lambda_{2} \geq \cdots \geq 0\right)$ is the largest integer $L(\lambda)$ such that $\lambda_{L(\lambda)} \neq 0$. The length of an $\ell$-partition $\lambda \in \mathcal{P}_{m}^{\Lambda}$ is $L(\lambda)=\max \left\{L\left(\lambda^{(l)}\right) \mid 1 \leq l \leq \ell\right\}$. If $\lambda \in \mathcal{P}_{n}^{\Lambda}$ then $L(\lambda) \leq n$ so, in what follows, we will always work with sequences of length $n$.

Following Littlewood [31, §2], the sequence $\beta_{1}^{\lambda}>\cdots>\beta_{n}^{\lambda} \geq 0$, where $\beta_{r}^{\lambda}=\lambda_{r}+n-r$, is a sequence of beta numbers of length $n$ for the partition $\lambda$. (If $\lambda$ is a partition of length $L=$ $L(\lambda)$ then the integers $\beta_{1}^{\lambda}+L-n>\cdots>\beta_{l}^{\lambda}+L-n$ are the first column hook lengths in $\lambda$.) More generally, a sequence of beta numbers is any strictly decreasing sequence $\beta_{1}>\cdots>\beta_{n} \geq 0$ of $n$ non-negative integers. It is straightforward to show that the set of beta numbers of length $n$ is in bijection with the set of partitions of length at most $n$, where a bijection is given by $\lambda \mapsto\left(\beta_{1}^{\lambda}>\cdots>\beta_{n}^{\lambda}\right)$. Notice that if $\left(\beta_{1}, \ldots, \beta_{n}\right)$ is a sequence of beta numbers of length $n$ then $\left(\beta_{1}+1, \ldots, \beta_{n}+1,0\right)$ is a sequence of beta numbers of length $n+1$.

Recall that we are assuming that the charge $\boldsymbol{\kappa}$ satisfies (3.7). A sequence of charged beta numbers (of length $\ell n$ ) is a sequence $\boldsymbol{\beta}=\left(\beta_{1}, \ldots, \beta_{\ell n}\right) \in \mathbb{N}^{\ell n}$ such that

$$
\kappa_{l}+n>\beta_{n(l-1)+1}>\cdots>\beta_{n l} \geq \kappa_{l}-n, \quad \text { for } 1 \leq l \leq \ell .
$$

That is, for $1 \leq l \leq n$ the subsequences $\left(\beta_{n(l-1)+1}, \ldots, \beta_{n l}\right)$ are beta numbers for the partitions $\lambda^{(l)}$ shifted by $\kappa_{l}-n \geq 0$. Assumption (3.7) ensures that $\beta_{1}>\cdots>\beta_{\ell n} \geq 0$ and, in particular, that the charged beta numbers are distinct. 
We frequently need to translate between $\ell$-partitions and charged beta numbers. To facilitate this, given an integer $s$ such that $1 \leq s \leq \ell n$ write $s=\left(l_{s}-1\right) n+r_{s}$, where $1 \leq l_{s} \leq \ell$ and $1 \leq r_{s} \leq n$. Similarly, if $1 \leq t \leq \ell n$ then we write $t=\left(l_{t}-1\right) n+r_{t}$ etc. In this way, we refer to row $s$ of $\lambda$ as row $r_{s}$ of $\lambda^{\left(l_{s}\right)}$. Of course, row $s$ of $\lambda$ is empty if $r_{s}>L\left(\lambda^{\left(l_{s}\right)}\right)$ or, equivalently, $\lambda_{r_{s}}^{\left(l_{s}\right)}=0$.

As with the beta numbers of partitions, if $\lambda \in \mathcal{P}_{m}^{\Lambda}$ and $n \geq L(\lambda)$ then $\lambda$ can be associated with a unique sequence of charged beta numbers of a given length. Explicitly, $\lambda \in \mathcal{P}_{m}^{\Lambda}$ has sequence of charged beta numbers $\boldsymbol{\beta}^{\lambda}=\left(\beta_{1}^{\lambda}, \ldots, \beta_{\ell n}^{\lambda}\right)$, where

$$
\beta_{s}^{\lambda}=\kappa_{l_{s}}+\lambda_{r_{s}}^{\left(l_{s}\right)}-r_{s}, \quad \text { where } s=\left(l_{s}-1\right) n+r_{s} \text { as above. }
$$

Even though our notation does not reflect this, $\boldsymbol{\beta}^{\lambda}=\boldsymbol{\beta}^{\lambda}(\boldsymbol{\kappa}, n)$ depends on $\lambda$ and the choice of $n$ and $\kappa$. When the order is not important, call $\left\{\beta_{1}^{\lambda}, \ldots, \beta_{\ell n}^{\lambda}\right\}$ a set of (charged) beta numbers for $\lambda$.

Via (5.2), the beta number $\beta_{s}^{\lambda}$ in (5.2) is naturally associated with the node $\left(l_{s}, r_{s}, \lambda_{r_{s}}^{\left(l_{s}\right)}\right)$ since, by definition, the content of the node is $\mathrm{c}\left(l_{s}, r_{s}, \lambda_{r_{s}}^{\left(l_{s}\right)}\right)=\beta_{s}^{\lambda}$. Notice that the node $\left(l_{s}, r_{s}, \lambda_{r_{s}}^{\left(l_{s}\right)}\right)$ belongs to $\lambda$ only if $L\left(\lambda^{\left(l_{s}\right)}\right) \geq r_{s}$.

If $\lambda \in \mathcal{P}_{m}^{\Lambda}$ then $\sum_{r=1}^{\ell n} \beta_{r}^{\lambda}=n\left(\kappa_{1}+\cdots+\kappa_{\ell}\right)-\frac{1}{2} \ell n(n+1)+m$. Even though we do not need this (although compare Definition 5.4), it is not difficult to see that there is a bijection between the $\ell$-partitions in $\mathcal{P}_{m}^{\Lambda}$ of length at most $n$ and the sequences of charged beta numbers with sum $n\left(\kappa_{1}+\cdots+\kappa_{\ell}\right)-\frac{1}{2} \ell n(n+1)+m$.

Example 5.3 Let $e=3$ and $\Lambda=\Lambda_{0}+\Lambda_{2}$, with $r(1,1,1)=0$. Let $\lambda=(3,2 \mid 1)$ so that $m=|\lambda|=6$. The following table lists some of the infinitely many choices of sequence of charged beta numbers for $\lambda$, corresponding to different choices of $\kappa$ and $n \geq L(\lambda)=2$.

\begin{tabular}{lll}
\hline $\mathrm{n}$ & $\kappa$ & $\beta^{\lambda}$ \\
\hline 2 & $(6,2)$ & $(8,6,2,0)$ \\
3 & $(12,5)$ & $(14,12,9,5,3,2)$ \\
4 & $(15,5)$ & $(17,15,12,11,5,3,2,1)$ \\
5 & $(15,5)$ & $(17,15,12,11,10,5,3,2,1,0)$ \\
6 & $(21,8)$ & $(23,21,18,17,16,15,8,6,5,4,3,2)$ \\
\hline
\end{tabular}

Notice that $\kappa$ must be chosen in accordance with (3.7), so it depends on $e, n$ and $\Lambda$, and that $\beta^{\lambda}$ depends on $\lambda$ and $\kappa$. Below we will assume that $n \geq m$, as in the last two example beta sequences, because this ensures that all partitions in $\mathcal{P}_{m}^{\Lambda}$ have a charged beta sequence of length $\ell n$. Consequently, these sequences of beta numbers can describe all possible ways of moving hooks (see Sect. 5.3), between the components of $\lambda$.

The main results in this chapter are proved by induction on the size $m$ of the $\ell$-partitions using charged beta sequences of length $\ell n$. Accordingly, we work with an $\ell$-partition $\lambda \in \mathcal{P}_{m}^{\Lambda}$, and use the sequence of charged beta numbers $\beta^{\lambda}$ defined in (5.2), which has length $\ell n$ where $n \geq m$.

If $\mathbf{i}=\left(i_{1}, \ldots, i_{n}\right) \in I^{m}$ and $1 \leq k \leq m$ let $\mathbf{i}_{\downarrow k}=\left(i_{1}, \ldots, i_{k}\right) \in I^{k}$. If $\boldsymbol{\beta} \in \mathbb{N}^{\ell n}$ and $w \in \mathfrak{S}_{\ell n}$ then let $w \cdot \boldsymbol{\beta}=\left(\beta_{w(1)}, \ldots, \beta_{w(\ell n)}\right)$. Since sequence of charged beta numbers are monotonically decreasing, $w \cdot \boldsymbol{\beta}$ is a sequence of charged beta numbers if and only if $w=1$. Abusing notation slightly, let $L(w)$ be the minimal length of $w \in \mathfrak{S}_{\ell n}$ as a product of the standard Coxeter generators of $\mathfrak{S}_{\ell n}$. 
Definition 5.4 Suppose that $\mathbf{i} \in I^{m}$ and $\boldsymbol{\beta}=\left(\beta_{1}, \ldots, \beta_{\ell n}\right) \in \mathbb{Z}^{\ell n}$, where $n \geq m$. Define $d_{\mathbf{i}}(\boldsymbol{\beta})= \begin{cases}(-1)^{L(w)} \operatorname{dim} S_{\mathbf{i}}^{\lambda}, & \text { if } \boldsymbol{\beta}=w \cdot \boldsymbol{\beta}^{\lambda} \text { for some } \lambda \in \mathcal{P}_{m}^{\Lambda} \text { and some } w \in \mathfrak{S}_{\ell n}, \\ 0, & \text { otherwise, }\end{cases}$ where $S_{\mathbf{i}}^{\lambda}=S_{F}^{\lambda} f_{\mathbf{i}}$. Similarly, set $d_{\mathbf{i}}(\lambda)=\operatorname{dim} S_{\mathbf{i}}^{\lambda}$.

In particular, $d_{\mathbf{i}}(\beta)=0$ if $\boldsymbol{\beta}=w \cdot \boldsymbol{\beta}^{\lambda}$ and $S_{\mathbf{i}}^{\lambda}=0$ for some $w \in \mathfrak{S}_{\ell n}$, or if $\beta_{s}=\beta_{t}$ for $s \neq t$, or if $\#\left\{1 \leq r \leq \ell n \mid \kappa_{l}+n>\beta_{r} \geq \kappa_{l}-n\right\} \neq n$, for any $l$ with $1 \leq l \leq \ell$.

\subsection{Beta numbers and branching rules}

The classical branching rules for the Specht modules of the symmetric groups are one of the cornerstones of modern representation theory. Analogues of these branching rules exist for the Specht modules of $\mathscr{H}_{n}^{\Lambda}[15,37]$. For this paper we only need the following simple consequence of these branching rules.

Let $\lambda \in \mathcal{P}_{n}^{\Lambda}$ and $\boldsymbol{v} \in \mathcal{P}_{n-1}^{\Lambda}$. Write $\boldsymbol{v} \rightarrow \lambda$ if $\lambda=\boldsymbol{v} \cup\{\alpha\}$ for some addable node $\alpha$ of $\boldsymbol{v}$. If $\alpha$ is an $i$-node, for $i \in I$ then we write $\boldsymbol{v} \stackrel{i}{\rightarrow} \boldsymbol{\mu}$ to emphasise the residue. If $\mathbf{j} \in I^{n-1}$ and $i \in I$ let $\mathbf{j} \vee i=\left(j_{1}, \ldots, j_{n-1}, i\right) \in I^{n}$. Extend $\_\vee i$ to a linear map $\mathbb{Z}\left[I^{n-1}\right] \hookrightarrow \mathbb{Z}\left[I^{n}\right]$.

Lemma 5.5 Suppose that $\lambda \in \mathcal{P}_{n}^{\Lambda}$. Then $\operatorname{ch} S_{F}^{\lambda}=\sum_{i \in I} \sum_{v \stackrel{i}{\rightarrow} \lambda} \operatorname{ch~} S_{F}^{v} \vee i$.

Proof By the main theorem of [37], the Specht module $S_{F}^{\lambda}$ has a filtration as an $\mathscr{H}_{n-1}^{\Lambda}(F)$ module where the quotients are exactly the Specht modules $S_{F}^{v}$, where $\boldsymbol{v} \rightarrow \lambda$, with each Specht module appearing exactly once. As the character map ch $: \operatorname{Rep} \mathscr{H}_{n-1}^{\Lambda} \longrightarrow \operatorname{Rep} \mathscr{L}_{n-1}$ is exact the result follows.

The advantage of using charged beta numbers, instead of $\ell$-partitions, is that it is much easier to describe induction and restriction. The next corollary uses charged beta numbers to give an equivalent version of Lemma 5.5 that is easier to apply because the beta numbers encode the relation $\boldsymbol{v} \rightarrow \lambda$.

Corollary 5.6 Suppose that $\mathbf{i} \in I^{m}$ and $\boldsymbol{\beta}=\left(\beta_{1}, \ldots, \beta_{\ell n}\right) \in \mathbb{N}^{\ell n}$, where $n \geq m$. Then

$$
d_{\mathbf{i}}(\boldsymbol{\beta})=\sum_{\substack{1 \leq s \leq \ell n \\ \beta_{s} \equiv i_{m}(\bmod e)}} d_{\mathbf{i}^{\prime}}\left(\beta_{1}, \ldots, \beta_{s-1}, \beta_{s}-1, \beta_{s+1}, \ldots, \beta_{\ell n}\right),
$$

where $\mathbf{i}^{\prime}=\mathbf{i}_{\downarrow(m-1)}$.

Proof First suppose that $\boldsymbol{\beta}=\boldsymbol{\beta}^{\lambda}$, for some $\ell$-partition $\lambda \in \mathcal{P}_{m}^{\Lambda}$. It is well-known and easy to see that removing a node from a $\lambda$ corresponds to decreasing one of the charged beta numbers of $\lambda$ by 1 . Now, $\boldsymbol{\beta}^{\prime}=\left(\beta_{1}, \ldots, \beta_{s-1}, \beta_{s}-1, \beta_{s+1}, \ldots, \beta_{\ell n}\right)$ is a sequence of charged beta numbers if and only if either $\beta_{s}>\kappa_{l}$ and $n \mid s$, or $\beta_{s}>\beta_{s+1}+1$ and $n \nmid s$. As above, write $s=\left(l_{s}-1\right) n+r_{s}$, where $1 \leq l_{s} \leq \ell$ and $1 \leq r_{s} \leq n$, then this corresponds to removing the node $\left(l_{s}, r_{s}, \lambda_{r_{s}}^{\left(l_{s}\right)}\right)$ from the $\ell$-partition corresponding to $\boldsymbol{\beta}$. When $\boldsymbol{\beta}=\boldsymbol{\beta}^{\lambda}$ the result now follows in view of Lemma 5.5. Finally, if $\boldsymbol{\beta}=w \cdot \boldsymbol{\beta}^{\lambda}$, for $w \in \mathfrak{S}_{\ell n}$, then it is easy to see that $\boldsymbol{\beta}^{\prime}$ is a sequence charged beta numbers if and only if $\boldsymbol{\beta}^{\prime}=w \cdot \boldsymbol{\beta}^{\boldsymbol{v}}$, for some $\ell$-partition $\boldsymbol{v} \in \mathcal{P}_{m-1}^{\Lambda}$, which implies the result. 


\subsection{Beta numbers and hooks}

This section shows that adding and subtracting numbers in a sequence of charged beta numbers corresponds to adding and removing rim hooks from the corresponding $\ell$-partitions. In order to describe this recall that if $\lambda$ is a partition then $\lambda^{\prime}=\left(\lambda_{1}^{\prime}, \lambda_{2}^{\prime}, \ldots\right)$ is the partition that is conjugate to $\lambda$, where $\lambda_{c}^{\prime}=\#\left\{r \geq 1 \mid \lambda_{r} \geq c\right\}$. Equivalently, $\lambda_{c}^{\prime}$ is the length of column $c$ of $\lambda$.

If $\alpha=(l, a, b) \in \lambda$ then the $\alpha$-rim hook of $\lambda$ is the following set of nodes:

$$
R_{\alpha}^{\lambda}=\left\{(l, c, d) \in \lambda \mid a \leq c \leq \lambda_{b}^{(l) \prime}, b \leq d \leq \lambda_{a}^{(l)}, \text { and }(l, c+1, d+1) \notin \lambda\right\} .
$$

By definition, $\lambda \backslash R_{\alpha}^{\lambda}$ is (the diagram of) an $\ell$-partition. The $\alpha$-hook length of $R_{\alpha}^{\lambda}$ is $h_{\alpha}=\left|R_{\alpha}^{\lambda}\right|$ and

$$
l_{\alpha}=\#\left\{(l, c, d) \in R_{\alpha}^{\lambda} \mid c>a\right\}=\lambda_{b}^{(l) \prime}-a
$$

is the $\alpha$-leg length of $R_{\alpha}^{\lambda}$. Let $r_{\alpha}=n(l-1)+a$ be the row of $\lambda$ that contains $\alpha$. The foot of $R_{\alpha}^{\lambda}$ is the node $f_{\alpha}=\left(l, a+l_{\alpha}, b\right)=\left(l, \lambda_{b}^{(l) \prime}, b\right) \in \lambda$, which is the leftmost node in the last row of $R_{\alpha}^{\lambda}$. Finally, an $h$-hook is any rim hook of length $h$.

Example 5.7 Let $\lambda=(4,2,1|6,5,3,2|, 3,1,1), \alpha=(2,1,3)$ and $n=m=28$. Then $f_{\alpha}=(2,3,3), h_{\alpha}=6, l_{\alpha}=2$ and $r_{\alpha}=n+1=29$. The rim hook $R_{\alpha}^{\lambda}$ can be pictured as follows:

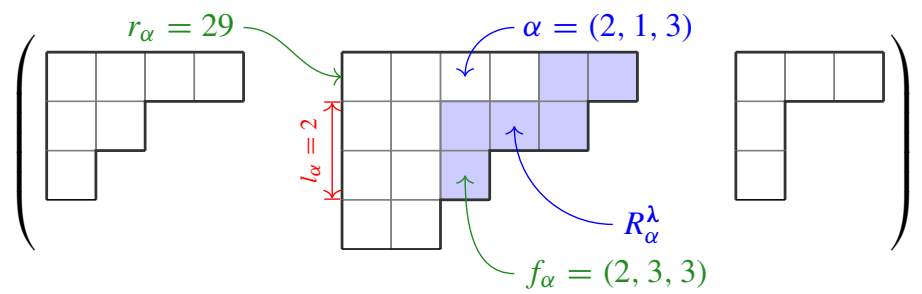

Definition 5.8 Let $\lambda \in \mathcal{P}_{m}^{\Lambda}$ and suppose that $\alpha=\left(l_{s}, r_{s}, c_{s}\right) \in \lambda$ and that $t>r_{\alpha}+l_{\alpha}=$ $(l-1) n+\lambda_{b}^{(l)^{\prime}}$, where $\lambda^{(l)^{\prime}}$ is the partition conjugate to $\lambda^{(l)}$. Define $\lambda_{\alpha, t}$ to be the $\ell$-partition of $m$ obtained by wrapping a rim hook of length $h_{\alpha}$ onto $\lambda \backslash R_{\alpha}^{\lambda}$ so that its foot node is the leftmost addable node in row $t$. If $\lambda_{\alpha, t}$ is not an $\ell$-partition, set $\lambda_{\alpha, t}=\emptyset$. If $\lambda_{\alpha, t} \neq \emptyset$ then set $\varepsilon_{\alpha, t}=(-1)^{l_{\alpha}+l_{\alpha^{\prime}}+1}$, where $\alpha^{\prime} \in \lambda_{\alpha, t}$ is the unique node such that $\lambda_{\alpha, t} \backslash R_{\alpha^{\prime}, t}^{\lambda_{\alpha, t}}=\lambda \backslash R_{\alpha}^{\lambda}$. Finally, set $h_{\alpha}^{\prime}=\beta_{s}^{\lambda}-\beta_{t}^{\lambda}-h_{\alpha}$, where $s=\left(l_{s}-1\right) n+r_{s}=r_{\alpha}$.

By definition, if $\alpha \in \lambda$ then the integer $h_{\alpha}^{\prime}$ depends on $\lambda, \kappa$ and $n \geq m$. Notice also that if $\lambda_{\alpha, t}=\emptyset$ then $\operatorname{ch} S_{F}^{\lambda_{\alpha, t}}=0$.

Example 5.9 Let $\lambda=(3,2 \mid 1) \in \mathcal{P}_{6}^{\Lambda}$. As in Example 5.3, take $n=m=6$ and $\kappa=(20,6)$ so that $\boldsymbol{\beta}^{\lambda}=(23,21,18,17,16,15,8,6,5,4,3,2)$. Using the notation of Definition 5.8, the following table gives the complete list of pairs $(\alpha, t)$ where $t>s$. Here, as in Definition 5.8, if $\alpha=\left(l_{s}, r_{s}, c_{s}\right)$ then $s=\left(l_{s}-1\right) n+r_{s}=r_{\alpha}$ and $h_{\alpha}^{\prime}=\beta_{s}^{\lambda}-\beta_{t}^{\lambda}-h_{\alpha}$. 


\begin{tabular}{|c|c|c|c|c|c|c|c|c|c|}
\hline & $\alpha$ & $\mathrm{t}$ & $\lambda_{\alpha, t}$ & & $\mathrm{~s}$ & $h_{\alpha}$ & $h_{\alpha^{\prime}}$ & $\varepsilon_{\alpha, t}$ & \\
\hline a) & $(1,1,3)$ & 3 & $\left(2^{2}, 1\right.$ & 1) & 1 & 1 & 4 & $(-1)^{0+0+1}=-1$ & $\mathrm{~h}$ \\
\hline b) & $(1,1,3)$ & 7 & $\left(2^{2}\right.$ & 2) & 1 & 1 & 14 & $(-1)^{0+0+0}=1$ & \\
\hline c) & $(1,1,3)$ & 8 & $\left(2^{2}\right.$ & $\left.1^{2}\right)$ & 1 & 1 & 16 & $(-1)^{0+0+0}=1$ & \\
\hline d) & $(1,1,2)$ & 5 & $\left(1^{5}\right.$ & 1) & 1 & 3 & 4 & $(-1)^{1+2+1}=1$ & i \\
\hline e) & $(1,1,2)$ & 7 & $\left(1^{2}\right.$ & 4) & 1 & 3 & 12 & $(-1)^{1+0+0}=-1$ & \\
\hline f) & $(1,1,2)$ & 8 & $\left(1^{2}\right.$ & $\left.2^{2}\right)$ & 1 & 3 & 14 & $(-1)^{1+1+0}=1$ & \\
\hline g) & $(1,1,2)$ & 10 & $\left(1^{2}\right.$ & $\left.1^{4}\right)$ & 1 & 3 & 16 & $(-1)^{1+2+0}=-1$ & \\
\hline h) & $(1,1,1)$ & 3 & $\left(2^{2}, 1\right.$ & 1) & 1 & 4 & 1 & $(-1)^{1+2+1}=1$ & $\mathrm{a}$ \\
\hline i) & $(1,1,1)$ & 5 & $\left(1^{5}\right.$ & 1) & 1 & 4 & 3 & $(-1)^{1+3+1}=-1$ & $\mathrm{~d}$ \\
\hline j) & $(1,1,1)$ & 7 & (1 & 5) & 1 & 4 & 11 & $(-1)^{1+0+0}=-1$ & \\
\hline k) & $(1,1,1)$ & 8 & $(1$ & $3,2)$ & 1 & 4 & 13 & $(-1)^{1+1+0}=1$ & \\
\hline 1) & $(1,1,1)$ & 9 & $(1$ & $\left.2^{2}, 1\right)$ & 1 & 4 & 14 & $(-1)^{1+2+0}=-1$ & \\
\hline $\mathrm{m})$ & $(1,1,1)$ & 11 & $(1$ & $\left.1^{5}\right)$ & 1 & 4 & 16 & $(-1)^{1+3+0}=1$ & \\
\hline n) & $(1,2,2)$ & 3 & $\left(3,1^{2}\right.$ & 1) & 2 & 1 & 2 & $(-1)^{0+0+1}=-1$ & $\mathrm{q}$ \\
\hline o) & $(1,2,2)$ & 7 & $(3,1$ & 2) & 2 & 1 & 12 & $(-1)^{0+0+0}=1$ & \\
\hline p) & $(1,2,2)$ & 8 & $(3,1$ & $\left.1^{2}\right)$ & 2 & 1 & 14 & $(-1)^{0+0+0}=1$ & \\
\hline q) & $(1,2,1)$ & 3 & $\left(3,1^{2}\right.$ & 1) & 2 & 2 & 1 & $(-1)^{0+1+1}=1$ & $\mathrm{n}$ \\
\hline r) & $(1,2,1)$ & 7 & $(3$ & 3) & 2 & 2 & 11 & $(-1)^{0+0+0}=1$ & \\
\hline s) & $(1,2,1)$ & 9 & $(3$ & $\left.1^{3}\right)$ & 2 & 2 & 14 & $(-1)^{0+1+0}=-1$ & \\
\hline
\end{tabular}

The right hand column identifies when a 2-partition appears more than once in the table as $\lambda_{\alpha, t}$, for some $\alpha$ and $t$. Proposition 5.11 below shows that no 2-partition appears more than twice and that if $\lambda_{\alpha, t}=\lambda_{\gamma, u}$, for some $\alpha, \gamma \in \lambda$ and some integers $t, u$, then $\left\{h_{\alpha}, h_{\alpha}^{\prime}\right\}=$ $\left\{h_{\gamma}, h_{\gamma}^{\prime}\right\}$ and $\varepsilon_{\alpha, t}=-\varepsilon_{\gamma, u}$.

Given $\lambda \in \mathcal{P}_{n}^{\Lambda}$ and two integers $1 \leq s, t \leq \ell n$, define the sequence:

$$
\boldsymbol{\beta}_{s t}^{\lambda}(h)=\left(\beta_{1}^{\lambda}, \ldots, \beta_{s-1}^{\lambda}, \beta_{s}^{\lambda}-h, \beta_{s+1}^{\lambda}, \ldots, \beta_{t-1}^{\lambda}, \beta_{t}^{\lambda}+h, \beta_{t+1}^{\lambda}, \ldots, \beta_{\ell n}^{\lambda}\right) \in \mathbb{Z}^{\ell n} .
$$

Such sequences will be used heavily in what follows. The next result records the combinatorial properties of sequences of charged beta numbers that we need.

Proposition 5.11 Let $\lambda \in \mathcal{P}_{m}^{\Lambda}$, where $n \geq m$ and fix $\mathbf{i} \in I^{m}$ and integers $1 \leq s<t \leq \ell$. Suppose that $d_{\mathbf{i}}\left(\boldsymbol{\beta}_{s t}^{\lambda}(h)\right) \neq 0$, where $h>0$, and set $h^{\prime}=\beta_{s}^{\lambda}-\beta_{t}^{\lambda}-h$.

(a) We have $h \neq h^{\prime}$ and $d_{\mathbf{i}}\left(\boldsymbol{\beta}_{s t}^{\lambda}\left(h^{\prime}\right)\right)=-d_{\mathbf{i}}\left(\boldsymbol{\beta}_{s t}^{\lambda}(h)\right)$. In particular, $d_{\mathbf{i}}\left(\boldsymbol{\beta}_{s t}^{\lambda}\left(h^{\prime}\right)\right) \neq 0$.

(b) If $h \leq m$ then $h=h_{\alpha}, h^{\prime}=h_{\alpha}^{\prime}$ and $d_{\mathbf{i}}\left(\boldsymbol{\beta}_{s t}^{\lambda}(h)\right)=-\varepsilon_{\alpha, t} d_{\mathbf{i}}\left(\lambda_{\alpha, t}\right)$, for some $\alpha \in \lambda$.

(c) If $h>m$ then $l_{s}<l_{t}$ and $h^{\prime} \leq m$. Moreover, $h^{\prime}=h_{\alpha^{\prime}}, h=h_{\alpha^{\prime}}^{\prime}$ and $d_{\mathbf{i}}\left(\boldsymbol{\beta}_{s t}^{\lambda}(h)\right)=$ $\varepsilon_{\alpha^{\prime}, t} d_{\mathbf{i}}\left(\lambda_{\alpha^{\prime}, t}\right)$, for some $\alpha^{\prime} \in \lambda$.

(d) If $\boldsymbol{\mu} \in \mathcal{P}_{m}^{\Lambda}$ and $d_{\mathbf{i}}(\boldsymbol{\mu})= \pm d_{\mathbf{i}}\left(\boldsymbol{\beta}_{s t}^{\lambda}(h)\right)$ then $\#\left\{(\alpha, t) \mid \boldsymbol{\mu}=\lambda_{\alpha, t}\right\}=\delta_{l_{s} l_{t}}+1$.

Proof By definition, $\beta_{s}^{\lambda}-h^{\prime}=\beta_{t}^{\lambda}+h$ and $\beta_{t}^{\lambda}+h^{\prime}=\beta_{t}^{\lambda}-h$. That is, $\boldsymbol{\beta}_{s t}^{\lambda}\left(h^{\prime}\right)=(s, t) \cdot \boldsymbol{\beta}_{s t}^{\lambda}(h)$, which implies that $d_{\mathbf{i}}\left(\boldsymbol{\beta}_{s t}^{\lambda}\left(h^{\prime}\right)\right)=-d_{\mathbf{i}}\left(\boldsymbol{\beta}_{s t}^{\lambda}(h)\right) \neq 0$. If $h=h^{\prime}$ this forces $d_{\mathbf{i}}\left(\boldsymbol{\beta}_{s t}^{\lambda}(h)\right)=0$, which is a contradiction. Hence, $h \neq h^{\prime}$ completing the proof of part (a).

Before considering (b) and (c) recall that going back to Littlewood (cf. [33, Lemma 5.26]), it is well-known that removing an $h$-rim hook from a partition corresponds to subtracting $h$ from one of the beta numbers of the partition. This result easily translates into an analogous 
result for $\ell$-partitions. Explicitly, if $\alpha \in \lambda$ then $\mu=\lambda \backslash R_{\alpha}^{\lambda}$ if and only if

$$
\beta_{k}^{\mu}= \begin{cases}\beta_{k+1}^{\lambda}, & \text { if } r_{\alpha}<k<r_{\alpha}+l_{\alpha}, \\ \beta_{r_{\alpha}}^{\lambda}-h_{\alpha}, & \text { if } r=r_{\alpha}+l_{\alpha}, \\ \beta_{k}^{\lambda}, & \text { otherwise. }\end{cases}
$$

Hence, if $\mathbf{j} \in I^{m-h_{\alpha}}$ then $d_{\mathbf{j}}(\boldsymbol{\mu})=(-1)^{l_{\alpha}} d_{\mathbf{j}}\left(\beta_{1}^{\lambda}, \ldots, \beta_{r_{\alpha}}^{\lambda}-h_{\alpha}, \ldots, \beta_{\ell n}^{\lambda}\right)$. In turn, this implies that if $\boldsymbol{v} \backslash R_{\alpha^{\prime}}^{v}=\lambda$ then $d_{\mathbf{k}}(\boldsymbol{v})=(-1)^{l_{\alpha^{\prime}}} d_{\mathbf{k}}\left(\beta_{1}^{\lambda}, \ldots, \beta_{r_{\alpha}}^{\lambda}+h_{\alpha^{\prime}}, \ldots, \beta_{\ell n}^{\lambda}\right)$, for $\mathbf{k} \in I^{m+h_{\alpha^{\prime}}}$. Consequently, if $\alpha \in \lambda$ and $\mathbf{i} \in I^{m}$ then $d_{\mathbf{i}}\left(\boldsymbol{\beta}_{s t}^{\lambda}\left(h_{\alpha}\right)\right)=(-1)^{l_{\alpha}+l_{\alpha^{\prime}}} d_{\mathbf{i}}\left(\boldsymbol{\lambda}_{\alpha, t}\right) .=-\varepsilon_{\alpha, t} d_{\mathbf{i}}\left(\lambda_{\alpha, t}\right)$. In particular, it follows that $h \leq m$ if and only if $h=h_{\alpha}$ for some $\alpha \in \lambda$, so we have proved (b) since $h_{\alpha}^{\prime}=\beta_{s}-\beta_{t}-h_{\alpha}=h^{\prime}$.

Next consider (c) and suppose that $h>m$. Then $\left\{\beta_{l_{s}+1}^{\lambda}, \ldots, \beta_{s}^{\lambda}-h, \ldots, \beta_{l_{s}+n-1}^{\lambda}\right\}$ cannot be a set of (shifted) beta numbers for $\lambda^{\left(l_{s}\right)}$ so $l_{s}<l_{t}$ and, as in the first paragraph, $\left\{\beta_{l_{s}+1}^{\lambda}, \ldots, \beta_{t}^{\lambda}+h^{\prime}, \ldots, \beta_{l_{s}+n-1}^{\lambda}\right\}$ and $\left\{\beta_{l_{t}+1}^{\lambda}, \ldots, \beta_{s}^{\lambda}-h^{\prime}, \ldots, \beta_{l_{t}+n-1}^{\lambda}\right\}$ are (shifted) sets of beta numbers for $\lambda^{\left(l_{s}\right)}$ and $\lambda^{\left(l_{t}\right)}$, respectively. Hence, by the last paragraph, $h^{\prime}=h_{\alpha^{\prime}}$ for some $\alpha^{\prime} \in \lambda$. In particular, $h^{\prime} \leq m$. Moreover, by parts (a) and (b), $d_{\mathbf{i}}\left(\boldsymbol{\beta}_{s t}^{\lambda}(h)\right)=-d_{\mathbf{i}}\left(\boldsymbol{\beta}_{s t}^{\lambda}\left(h^{\prime}\right)\right)=$ $\varepsilon_{\alpha^{\prime}, t} d_{\mathbf{i}}\left(\lambda_{\alpha^{\prime}, t}\right)$. This completes the proof of (c).

Finally, as in (d), suppose that $\boldsymbol{\mu} \in \mathcal{P}_{m}^{\Lambda}$ and that $d_{\mathbf{i}}(\boldsymbol{\mu})= \pm d_{\mathbf{i}}\left(\boldsymbol{\beta}_{s t}^{\lambda}(h)\right)$. Then, there exist $s^{\prime}<t^{\prime}$ and $h>0$ such that $\left\{\beta_{r}^{\lambda} \mid r \neq s, t\right\}=\left\{\beta_{r}^{v} \mid r \neq s^{\prime}, t^{\prime}\right\}$ and

$$
\left\{\beta_{s^{\prime}}^{v}, \beta_{t^{\prime}}^{\nu}\right\}=\left\{\beta_{s}^{\lambda}-h, \beta_{t}^{\lambda}+h\right\}=\left\{\beta_{s}^{\lambda}+h^{\prime}, \beta_{t}^{\lambda}-h^{\prime}\right\},
$$

where $h^{\prime}=\beta_{s}^{\lambda}-\beta_{t}^{\lambda}-h$. In particular, there are exactly two ways to write $\mu$ in the form $\lambda_{\alpha, t}$ if $h \leq m$ and $h^{\prime} \leq m$. If $h>m$ or $h^{\prime}>m$ then, as in the last paragraph, there is only one to write $\boldsymbol{\mu}$ in this form and, moreover, $l_{s}<l_{t}$. Note that part (c) implies that if $h>m$ then $\boldsymbol{\mu}=\lambda_{\alpha^{\prime}, t}$ and $h^{\prime}=h_{\alpha^{\prime}} \leq m$, for some $\alpha^{\prime} \in \lambda$. Similarly, if $h^{\prime}>m$ then $\boldsymbol{\mu}=\lambda_{\alpha, t}$ and $h=h_{\alpha} \leq m$, for $\alpha \in \lambda$. Hence, (d) follows.

\subsection{Beta numbers and Gram determinants}

We can now prove our "classical" Jantzen sum formula. We start by giving a closed formula for the $\gamma$-coefficients from (3.11).

Given two nodes $\alpha=(m, r, c)$ and $\alpha^{\prime}=\left(m^{\prime}, r^{\prime}, c^{\prime}\right)$ define $\alpha$ to be below $\alpha^{\prime}$ if either $m^{\prime}<$ $m$ or $m^{\prime}=m$ and $c^{\prime}<c$. Let $\operatorname{Add}_{k}(\mathfrak{t})$ to be the set of addable nodes of the tableau $\mathfrak{t}_{\downarrow k}$ that are below the node $\mathfrak{t}^{-1}(k)$. Similarly, let $\operatorname{Rem}_{k}(\mathfrak{t})$ be the set of removable nodes of the tableau $\mathfrak{t}_{\downarrow k}$ that are below the node $\mathfrak{t}^{-1}(k)$.

Lemma 5.12 Suppose that $\mathfrak{t} \in \operatorname{Std}(\lambda)$, for $\lambda \in \mathcal{P}_{m}^{\Lambda}$. Then

$$
\gamma_{\mathfrak{t}}=z^{h_{\mathfrak{t}}} \prod_{k=1}^{m} \frac{\prod_{\alpha \in \operatorname{Add}_{k}(\mathfrak{t})}\left[c_{k}(\mathfrak{t})-c(\alpha)\right]_{z}}{\prod_{\beta \in \operatorname{Rem}_{k}(\mathfrak{t})}\left[c_{k}(\mathfrak{t})-c(\beta)\right]_{z}},
$$

for some $h_{\mathfrak{t}} \in \mathbb{Z}$.

Proof This follows by induction on the dominance order. The base case is given by the closed formula for $\gamma_{t^{\lambda}}$ given in (4.8) and the inductive step follows using Corollary 3.12. Compare with [19, Definition 3.15 and Proposition 3.19].

The next result is a "branching rule" for $\operatorname{det} G_{\mathbf{i}}^{\lambda}$ using the charged beta numbers. 
Lemma 5.13 Suppose that $\lambda \in \mathcal{P}_{m}^{\Lambda}$ and $\mathbf{i} \in I^{m}$. Let $\mathbf{i}^{\prime}=\mathbf{i}_{\downarrow}(m-1)$. Then there exists an integer

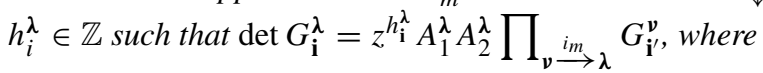

$$
\begin{aligned}
A_{1}^{\lambda} & =\prod_{h \geq 1} \prod_{\substack{1 \leq s<t \leq \ell n \\
\beta_{s}^{\lambda}-\beta_{t}^{\lambda}-1=h \\
\beta_{s}^{\lambda} \equiv i_{m}}}[h]_{z}^{-d_{\mathbf{i}^{\prime}}\left(\beta_{1}^{\lambda}, \ldots, \beta_{s}^{\lambda}-h-1, \ldots, \beta_{t}^{\lambda}+h, \ldots, \beta_{\ell n}^{\lambda}\right)} \\
\text { and } A_{2}^{\lambda} & =\prod_{h \geq 1} \prod_{\substack{1 \leq s<t \leq \ell n \\
\beta_{s}^{\lambda}-\beta_{t}^{\lambda}=h \\
\beta_{s}^{\lambda} \equiv i_{m}}}[h]_{z}^{d_{\mathbf{i}^{\prime}}\left(\beta_{1}^{\lambda}, \ldots, \beta_{s}^{\lambda}-h, \ldots, \beta_{t}^{\lambda}+h-1, \ldots, \beta_{\ell n}^{\lambda}\right)} .
\end{aligned}
$$

Proof If $m=1$ then there is nothing to prove, so assume $m>1$. By Lemma 3.21 and Lemma 5.12 ,

$$
\begin{aligned}
\operatorname{det} G_{\mathbf{i}}^{\lambda} & =\prod_{\mathfrak{t} \in \operatorname{Std}_{\mathbf{i}}(\lambda)} \gamma_{\mathfrak{t}}=\prod_{\mathfrak{t} \in \operatorname{Std}_{\mathbf{i}}(\lambda)} z^{h \mathfrak{t}} \prod_{k=1}^{m} \frac{\prod_{\alpha \in \operatorname{Add}_{k}(\mathfrak{t})}\left[\mathrm{c}_{k}(\mathfrak{t})-\mathrm{c}(\alpha)\right]_{z}}{\prod_{\beta \in \operatorname{Rem}_{k}(\mathfrak{t})}\left[c_{k}(\mathfrak{t})-\mathrm{c}(\beta)\right]_{z}} \\
& =z^{a} \prod_{\substack{i_{m} \\
\rightarrow \lambda}} z^{h_{\mathbf{i}^{\prime}}^{v}} G_{\mathbf{i}^{\prime}}^{v} \cdot \prod_{\mathfrak{t} \in \operatorname{Std}_{\mathbf{i}}(\lambda)} \frac{\prod_{\alpha \in \operatorname{Add}_{m}(\mathfrak{t})}\left[\mathbf{c}_{m}(\mathfrak{t})-\mathrm{c}(\alpha)\right]_{z}}{\prod_{\beta \in \operatorname{Rem}_{m}(\mathfrak{t})}\left[\mathrm{c}_{m}(\mathfrak{t})-\mathrm{c}(\beta)\right]_{z}}
\end{aligned}
$$

for some $a \in \mathbb{Z}$, since $\mathfrak{t}_{\downarrow(m-1)} \in \operatorname{Std}(\boldsymbol{v})$ for some $\boldsymbol{v} \stackrel{i_{m}}{\rightarrow} \lambda$, for all $\mathfrak{t} \in \operatorname{Std}_{\mathbf{i}}(\lambda)$. Set $h_{\mathbf{i}}^{\lambda}=$ $a+\sum_{v} h_{\mathbf{i}^{\prime}}^{v}$.

Fix $\mathfrak{t} \in \operatorname{Std}(\lambda)$ and consider its contribution to $\operatorname{det} G_{\mathbf{i}}^{\lambda}$ in the product above. As we read down the rows in each component, the nodes in $\operatorname{Add}_{m}(\mathfrak{t}) \cup \operatorname{Rem}_{m}(\mathfrak{t})$ alternate between addable and removable nodes, finishing with the addable node $\left(k, L\left(\lambda^{(j)}\right)+1,1\right)$ at the bottom of the $j$ th component, for $1 \leq j \leq \ell$. Set $\gamma=\mathfrak{t}^{-1}(m)=\left(l_{s}, r_{s}, a\right)$ and let $\beta=\left(l_{v}, r_{v}, c\right) \in$ $\operatorname{Rem}_{m}(\mathfrak{t})$ be a removable node and let $\alpha=\left(l_{u}, r_{u}, b\right)$ be the addable node directly above it. Write $s=\left(l_{s}-1\right) n+r_{s}, u=\left(l_{u}-1\right) n+r_{u}$ and $v=\left(l_{v}-1\right) n+r_{v}$. Note that $r_{v}>r_{u}$ since $\beta$ is below $\alpha$, so $v>u$. The consecutive beta numbers between rows $u$ and $v$ all differ by 1 , so he contribution from these three nodes to $G_{\mathbf{i}}^{\mu}$ is

$$
\frac{\left[c_{m}(\mathfrak{t})-c(\alpha)\right]_{z}}{\left[c_{m}(\mathfrak{t})-c(\beta)\right]_{z}}=\frac{\left[\beta_{s}^{\lambda}-\beta_{u}^{\lambda}-1\right]_{z}}{\left[\beta_{s}^{\lambda}-\beta_{v}^{\lambda}\right]_{z}}=\prod_{t=u}^{v} \frac{\left[\beta_{s}^{\lambda}-\beta_{t}^{\lambda}-1\right]_{z}}{\left[\beta_{s}^{\lambda}-\beta_{t}^{\lambda}\right]_{z}} .
$$

The addable nodes $\left(j, L\left(\lambda^{(j)}\right)+1,1\right) \in \operatorname{Add}_{m}(\mathfrak{t})$ at the bottom of $\lambda^{(j)}$, for $l \leq j \leq \ell$, have not yet been included in this calculation. Via $\mathfrak{t}$, the contribution of such nodes to $\operatorname{det} G_{\mathbf{i}}^{\lambda}$ is:

$$
\prod_{j=l}^{\ell}\left[c_{m}(\mathfrak{t})-\kappa_{j}+L\left(\lambda^{(j)}\right)\right]=\prod_{j=l+1}^{\ell}\left[\beta_{s}^{\lambda}-\beta_{j n}^{\lambda}\right]_{z} \prod_{t=(j-1) n+L\left(\lambda^{(j)}\right)+1}^{j n} \frac{\left[\beta_{s}^{\lambda}-\beta_{t}^{\lambda}-1\right]_{z}}{\left[\beta_{s}^{\lambda}-\beta_{t}^{\lambda}\right]_{z}} .
$$

For each $j$, all but one of the terms in the right hand product cancels out because $\beta_{t}^{\lambda}=\kappa_{j}-t$, for $(j-i) L\left(\lambda^{(j)}\right)<t \leq j n$. The point of adding the extra terms to the last two displayed equations is that this allows us to give a generic formula for these determinants.

If $\mathfrak{s}, \mathfrak{t} \in \operatorname{Std}_{\mathbf{i}}(\lambda)$ and $\boldsymbol{v}=\operatorname{Shape}\left(\mathfrak{s}_{\downarrow}(m-1)\right)=\operatorname{Shape}\left(\mathfrak{t}_{\downarrow(m-1)}\right)$ then $\operatorname{Add}_{m}(\mathfrak{s})=\operatorname{Add}_{m}(\mathfrak{t})$ and $\operatorname{Rem}_{m}(\mathfrak{s})=\operatorname{Rem}_{m}(\mathfrak{t})$, so such tableaux contribute the same factor to $\operatorname{det} G_{\mathbf{i}}^{\lambda}$. The number 
of these tableaux is $\operatorname{dim} S_{\mathbf{i}^{\prime}}^{\boldsymbol{v}}=d_{\mathbf{i}^{\prime}}\left(\beta_{1}^{\lambda}, \ldots, \beta_{s}^{\lambda}-1, \ldots, \beta_{\ell n}^{\lambda}\right)$, since $\mathfrak{t}^{-1}(m)=\left(l_{s}, r_{s}, a\right)$. Therefore, combining all of contributions to $G_{\mathbf{i}}^{\lambda}$ from all $\mathfrak{t} \in \operatorname{Std}_{\mathbf{i}}(\lambda)$ shows that

$$
\operatorname{det} G_{\mathbf{i}}^{\lambda}=z^{h_{\mathbf{i}}^{\lambda}} \prod_{\substack{i_{m} \\ \longrightarrow \lambda}} G_{\mathbf{i}^{\prime}}^{\boldsymbol{v}} \cdot \prod_{\substack{t=1 \\ \beta_{t}^{\lambda} \equiv i_{m}}}^{\ell n}\left(\frac{\prod_{t=s+1}^{\ell n}\left[\beta_{s}^{\lambda}-\beta_{t}^{\lambda}-1\right]_{z}}{\prod_{\substack{t=s+1 \\ n \nmid t}}^{\ell n}\left[\beta_{s}^{\lambda}-\beta_{t}^{\lambda}\right]_{z}}\right)^{d_{\mathbf{i}^{\prime}}\left(\beta_{1}^{\lambda}, \ldots, \beta_{s}^{\lambda}-1, \ldots, \beta_{t}^{\lambda}, \ldots, \beta_{\ell n}^{\lambda}\right),}
$$

In fact, in addition to the terms already identified, the right hand product above introduces extra terms when $\beta_{t}^{\lambda} \not \equiv i_{m}$ or when the node corresponding to $\beta_{t}^{\lambda}$ is not removable, in which case the exponent $d_{\mathbf{i}^{\prime}}\left(\beta_{1}^{\lambda}, \ldots, \beta_{s}^{\lambda}-1, \ldots, \beta_{\ell n}^{\lambda}\right)=0$. Hence, all of these "extra terms" either cancel out or are equal to 1 , so adding them to the product does not change the determinant. In addition, the condition $n \nmid t$ in the denominator can be omitted because all of the extra terms that this introduces to the denominator are equal to 1 since they all have exponent zero.

Write the right hand product as $A_{1}^{\lambda} / A_{2}^{\lambda}$. Setting $h=\beta_{s}^{\lambda}-\beta_{t}^{\lambda}-1$, the exponent of $[h]_{z}$ in $A_{1}^{\lambda}$ is

$$
\begin{aligned}
d_{\mathbf{i}^{\prime}}\left(\beta_{1}^{\lambda}, \ldots, \beta_{s}^{\lambda}-1, \ldots, \beta_{t}^{\lambda}, \ldots, \beta_{\ell n}^{\lambda}\right) & =d_{\mathbf{i}^{\prime}}\left(\beta_{1}^{\lambda}, \ldots, \beta_{t}^{\lambda}+h, \ldots, \beta_{s}^{\lambda}-h-1, \ldots, \beta_{\ell n}^{\lambda}\right) \\
& =-d_{\mathbf{i}^{\prime}}\left(\beta_{1}^{\lambda}, \ldots, \beta_{s}^{\lambda}-h-1, \ldots, \beta_{t}^{\lambda}+h, \ldots, \beta_{\ell n}^{\lambda}\right) .
\end{aligned}
$$

Similarly, setting $h=\beta_{s}^{\lambda}-\beta_{t}^{\lambda}$ in $A_{2}^{\lambda}$, the exponent $[h]_{z}$ in $A_{2}^{\lambda}$ is

$$
\begin{aligned}
& -d_{\mathbf{i}^{\prime}}\left(\beta_{1}^{\lambda}, \ldots, \beta_{t}^{\lambda}+h-1, \ldots, \beta_{s}^{\lambda}-h, \ldots, \beta_{\ell n}^{\lambda}\right)=d_{\mathbf{i}^{\prime}}\left(\beta_{1}^{\lambda}, \ldots, \beta_{s}^{\lambda}-h, \ldots, \beta_{t}^{\lambda}\right. \\
& \left.\quad+h-1, \ldots, \beta_{\ell n}^{\lambda}\right) .
\end{aligned}
$$

Combining the terms in the numerator and denominator in this way completes the proof.

Armed with the inductive formula of Lemma 5.13 we can now give our first closed formula for the Gram determinant $G_{\mathbf{i}}^{\lambda}$. This result should be compared with [19, Theorem 3.35].

Recall the definition of the sequence $\boldsymbol{\beta}_{s t}^{\lambda}(h)$ (5.10). Given $1 \leq s, t \leq \ell n$ define $\delta_{l_{s} l_{t}}(n)=$ $\left(1-\delta_{l_{s} l_{t}}\right) n$. That is, $\delta_{l_{s} l_{t}}(n)=0$ if $l_{s}=l_{t}$ and $\delta_{l_{s} l_{t}}(n)=n$ otherwise. Notice that the product formula in the next result does not require $s<t$ although, implicitly, it does require $l_{s} \leq l_{t}$.

Proposition 5.14 Let $\mathbf{i} \in I^{m}, \lambda \in \mathcal{P}_{m}^{\Lambda}$ and $n \geq m$. Then there exists $h_{\mathbf{i}}^{\lambda} \in \mathbb{Z}$ such that $t$

$$
\operatorname{det} G_{\mathbf{i}}^{\lambda}=z^{h_{\mathbf{i}}^{\lambda}} \prod_{h \geq 1} \prod_{\substack{1 \leq s, t \leq \ell n \\ \delta_{l_{s} l_{t}}(n)<h \leq \beta_{s}^{\lambda}-\beta_{t}^{\lambda}}}[h]_{z}^{d_{\mathbf{i}}\left(\boldsymbol{\beta}_{s t}^{\lambda}(h)\right)} .
$$

Proof We argue by induction on $m$. If $m=0$ then $\lambda=(0|\ldots| 0)$ and

$$
\boldsymbol{\beta}^{\lambda}=\left(\kappa_{1}-1, \kappa_{1}-2, \ldots, \kappa_{1}-n, \kappa_{2}-1, \ldots, \kappa_{2}-n, \ldots, \ldots, \kappa_{\ell}-n\right) .
$$

By convention empty products are 1 , so det $G_{\mathbf{i}}^{\lambda}=1$. Similarly, since $\delta_{l_{s} l_{t}}(n)=0$ for all $(s, t)$ the right hand product is equal to 1 in view of Proposition 5.11. Hence, the proposition holds when $m=0$, giving us the base case of our induction. 
Now suppose that $m>0$ and, as in Lemma 5.13, let $\mathbf{i}^{\prime}=\mathbf{i}_{\downarrow(m-1)}$. Recalling the notation and statement of Lemma 5.13 and applying induction,

$$
\begin{aligned}
& z^{-h_{\mathbf{i}}^{\lambda} \operatorname{det} G_{\mathbf{i}}^{\lambda}}=A_{1}^{\lambda} A_{2}^{\lambda} \prod \operatorname{det} G_{\mathbf{i}^{\prime}}^{v} \\
& \nu \stackrel{i_{m}}{\rightarrow} \lambda \\
& =A_{1}^{\lambda} A_{2}^{\lambda} \prod_{\nu} \prod_{\substack{i_{m} \\
\longrightarrow}} \prod_{\substack{1 \leq s, t \leq l n \\
\delta_{l_{s} l_{t}}(n)<h<\beta_{s}^{v}-\beta_{t}^{v}}}[h]_{z}^{d_{\mathbf{i}^{\prime}}\left(\beta_{1}^{v}, \ldots, \beta_{s}^{v}-h, \ldots, \beta_{t}^{v}+h, \ldots \beta_{\ell n}^{v}\right)} \\
& =\prod_{\substack{1 \leq r \leq \ell n \\
\beta_{r}^{\lambda} \equiv i_{m}}} \prod_{h \geq 1}\left\{\prod_{\substack{1 \leq s, t \leq \ell n, r \neq s, t \\
\delta_{l_{s} l_{t}}(n)<h<\beta_{s}^{\lambda}-\beta_{t}^{\lambda}}}[h]_{z}^{d_{\mathrm{i}^{\prime}}\left(\beta_{1}^{\lambda}, \ldots \ldots, \beta_{s}^{\lambda}-h, \ldots, \beta_{r}^{\lambda}-1, \ldots, \beta_{t}^{\lambda}+h, \ldots \beta_{\ell n}^{\lambda}\right)}\right. \\
& \times A_{1}^{\lambda} \prod_{\substack{1 \leq t \leq \ell n \\
\delta_{l_{s} l_{t}}(n)<h<\beta_{r}^{\lambda}-1-\beta_{t}^{\lambda}}}[h]_{z}^{d_{\mathrm{i}^{\prime}}\left(\beta_{1}^{\lambda}, \ldots, \beta_{r}^{\lambda}-1-h, \ldots, \beta_{t}^{\lambda}+h, \ldots \beta_{\ell n}^{\lambda}\right)} \\
& \left.\times A_{2}^{\lambda} \quad \prod_{1 \leq s \leq \ell n}[h]_{z}^{d_{\mathrm{i}^{\prime}}\left(\beta_{1}^{\lambda}, \ldots, \beta_{s}^{\lambda}-h, \ldots, \beta_{r}^{\lambda}-1+h, \ldots \beta_{\ell n}^{\lambda}\right)}\right\} \\
& \delta_{l_{s} l_{t}}(n)<h<\beta_{s}^{\lambda}-\beta_{r}^{\lambda}+1 \\
& =\prod_{\substack{1 \leq r \leq \ell n \\
\beta_{r}^{\lambda} \equiv i_{m}}} \prod_{h \geq 1}\left\{\prod_{\substack{1 \leq s, t \leq \ell n, r \neq s, t \\
\delta_{l_{s} l_{t}}(n)<h<\beta_{s}^{\lambda}-\beta_{t}^{\lambda}}}[h]_{z}^{d_{\mathrm{i}^{\prime}}\left(\beta_{1}^{\lambda}, \ldots \ldots, \beta_{s}^{\lambda}-h, \ldots, \beta_{r}^{\lambda}-1, \ldots, \beta_{t}^{\lambda}+h, \ldots \beta_{\ell n}^{\lambda}\right)}\right. \\
& \prod_{\substack{1 \leq t \leq \ell n \\
\delta_{l_{s} l_{t}}(n)<h<\beta_{r}^{\lambda}-1-\beta_{t}^{\lambda}}}[h]_{z}^{d_{\mathrm{i}^{\prime}}\left(\beta_{1}^{\lambda}, \ldots, \beta_{r}^{\lambda}-1-h, \ldots, \beta_{t}^{\lambda}+h, \ldots \beta_{\ell n}^{\lambda}\right)} \\
& \left.\prod_{\substack{1 \leq s \leq \ell n \\
\delta_{l_{s} l_{t}}(n)<h<\beta_{s}^{\lambda}-\beta_{r}^{\lambda}}}[h]_{z}^{d_{\mathbf{i}^{\prime}}\left(\beta_{1}^{\lambda}, \ldots, \beta_{s}^{\lambda}-h, \ldots, \beta_{r}^{\lambda}-1+h, \ldots \beta_{\ell n}^{\lambda}\right)}\right\},
\end{aligned}
$$

where the last equality follows using the formulas for $A_{1}^{\lambda}$ and $A_{2}^{\lambda}$ from Lemma 5.13. Applying Corollary 5.6 completes the proof.

Remark 5.15 The proof of Proposition 5.14, and the notation that it uses, is different from the results in $[18,19]$ because our definition of charged beta numbers corresponds to using first column hook lengths whereas these references use first row hook lengths. In the mathematics, this difference manifests itself through subtle sign changes. For example, [18] requires that $h>\beta_{s}^{\lambda}-\beta_{t}^{\lambda}$ whereas we require that $h<\beta_{s}^{\lambda}-\beta_{t}^{\lambda}$. We use column hook lengths because of their direct connection with beta numbers.

Example 5.16 Let $e=3, \Lambda=\Lambda_{0}+\Lambda_{2}$ and $\lambda=(3,2 \mid 1)$. As in Example 5.3 and Example 5.9 , take $n=6$ and $\kappa=(20,6)$ so that $\beta^{\lambda}=(23,21,18,17,16,15,8,6,5,4,3,2)$. The following table gives the different contributions to $\operatorname{det} G_{\mathbf{i}}^{\lambda}$, for $\mathbf{i} \in I^{n}$, given by Proposition 5.14. The entries in each row are completely determined by $\boldsymbol{\beta}^{\lambda}$ and the integers $s, t$ and $h$. In particular, $h^{\prime}=\beta_{s}^{\lambda}-\beta_{t}^{\lambda}-h$ and the $\ell$-partition $\boldsymbol{v}$ and the sign $\varepsilon= \pm 1$ are determined by $d_{\mathbf{i}}\left(\beta_{1}^{\lambda}, \ldots, \beta_{s}^{\lambda}-h, \ldots, \beta_{t}^{\lambda}+h, \ldots \beta_{\ell n}^{\lambda}\right)=\varepsilon d_{\mathbf{i}}(\boldsymbol{v})$. 


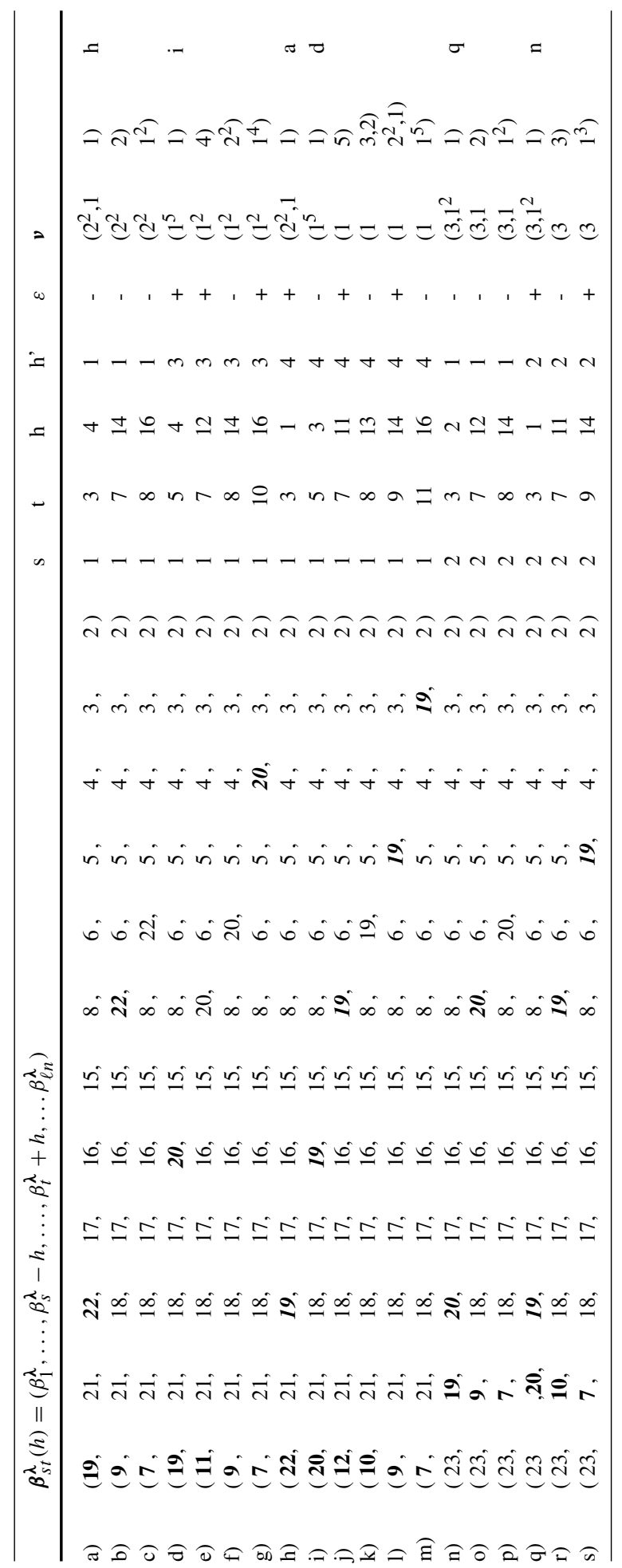


(The only purpose of the bold numbers is to highlight which beta numbers change. The row labels in the left hand column are to facilitate comparison with Example 5.9.) Hence, by Proposition 5.14,

$$
\begin{aligned}
& G_{\mathbf{i}}^{\lambda}=\left(\frac{[1]_{z}}{[4]_{z}}\right)^{d_{\mathbf{i}}\left(2^{2}, 1 \mid 1\right)}[14]_{z}^{d_{\mathbf{i}}\left(2^{2} \mid 2\right)}[16]_{z}^{d_{\mathbf{i}}\left(2^{2} \mid 1^{2}\right)}\left(\frac{[4]_{z}}{[3]_{z}}\right)^{d_{\mathbf{i}}\left(1^{5} \mid 1\right)} \\
& {[12]_{z}^{-d_{\mathbf{i}}\left(1^{2} \mid 4\right)}[14]_{z}^{d_{\mathbf{i}}\left(1^{2} \mid 2^{2}\right)}[16]_{z}^{-d_{\mathbf{i}}\left(1^{2} \mid 1^{4}\right)}[11]_{z}^{-d_{\mathbf{i}}(1 \mid 5)}} \\
& \times[13]_{z}^{d_{\mathbf{i}}(1 \mid 3,2)}[14]_{z}^{d_{\mathbf{i}}\left(1 \mid 2^{2}, 1\right)}[16]_{z}^{d_{\mathbf{i}}\left(1 \mid 1^{5}\right)}\left(\frac{[1]_{z}}{[2]_{z}}\right)^{d_{\mathbf{i}}\left(3,1^{2} \mid 1\right)} \\
& {[12]_{z}^{d_{\mathbf{i}}(3,1 \mid 2)}[14]_{z}^{-d_{\mathbf{i}}\left(3,1 \mid 1^{2}\right)}[11]_{z}^{d_{\mathbf{i}}(3 \mid 3)}[14]_{z}^{-d_{\mathbf{i}}\left(3 \mid 1^{3}\right)} .}
\end{aligned}
$$

By construction, $\operatorname{det} G_{\mathbf{i}}^{\lambda} \in \mathcal{O}$. By Lemma $4.6,[h]_{z}$ is a unit in $\mathcal{O}$ whenever $h \not \equiv 0(\bmod e)$. Therefore,

$$
G_{\mathbf{i}}^{\lambda}=u[3]_{z}^{-d_{\mathbf{i}}\left(1^{5} \mid 1\right)}[12]_{z}^{d_{\mathbf{i}}(3,1 \mid 2)-d_{\mathbf{i}}\left(1^{2} \mid 4\right)}=u[3]_{z}^{d_{\mathbf{i}}(3,1 \mid 2)-d_{\mathbf{i}}\left(1^{2} \mid 4\right)-d_{\mathbf{i}}\left(1^{5} \mid 1\right)}[4]_{z^{3}}^{d_{\mathbf{i}}(3,1 \mid 2)-d_{\mathbf{i}}\left(1^{2} \mid 4\right)},
$$

for some unit $u \in \mathcal{O}$.

Recall from Sect. 5.3 that $r_{\alpha}$ is the row index of the foot of the $\alpha$-hook $R_{\alpha}^{\lambda}$.

Corollary 5.17 Suppose that $\lambda \in \mathcal{P}_{n}^{\Lambda}$ and $\mathbf{i} \in I^{n}$. Then $\operatorname{det} G_{\mathbf{i}}^{\lambda}=\prod_{\alpha \in \lambda} \prod_{t>r_{\alpha}}\left[h_{\alpha}^{\prime}\right]_{z}^{\varepsilon_{\alpha, t} d_{\mathbf{i}}\left(\lambda_{\alpha, t}\right)}$.

Proof By Proposition 5.14, $G_{\mathbf{i}}^{\lambda}=\prod_{h, s, t}[h]_{z}^{d_{\mathbf{i}}\left(\beta_{s t}^{\lambda}(h)\right)}$ where, in the product, $\delta_{l_{s} l_{t}}(n)<h \leq$ $\beta_{s}^{\lambda}-\beta_{t}^{\lambda}$. By parts (b) and (c) of Proposition 5.11, if $\delta_{l_{s} l_{t}}(n)<h \leq \beta_{s}^{\lambda}-\beta_{t}^{\lambda}$ then there exists a node $\alpha \in \lambda$ such that $h=h_{\alpha}^{\prime}, t>r_{\alpha}$ and $d_{\mathbf{i}}\left(\boldsymbol{\beta}_{s t}^{\lambda}(h)\right)=\varepsilon_{\alpha, t} d_{\mathbf{i}}\left(\lambda_{\alpha, t}\right)$. Hence, the result follows.

We can now give the promised "classical" higher level Jantzen character formula.

Theorem 5.18 Suppose that $\lambda \in \mathcal{P}_{n}^{\Lambda}$. Then $\operatorname{ch} J_{F}^{\lambda}=\sum_{\alpha \in \lambda} \sum_{t>r_{\alpha}} \varepsilon_{\alpha, t} v_{x}\left(\left[h_{\alpha}^{\prime}\right]_{z}\right) \operatorname{ch} S_{F}^{\lambda_{\alpha, t}}$.

Proof Applying Proposition 4.3 and Corollary 5.17,

$$
\begin{aligned}
\operatorname{ch} J_{F}^{\lambda} & =\sum_{\mathbf{i} \in I^{n}} v_{x}\left(\operatorname{det} G_{\mathbf{i}}^{\lambda}\right) \mathbf{i}=\sum_{\mathbf{i} \in I^{n}}\left(\sum_{\alpha \in \lambda} \sum_{t>r_{\alpha}} \varepsilon_{\alpha, t} v_{x}\left(\left[h_{\alpha}^{\prime}\right]_{z}\right) d_{\mathbf{i}}\left(\lambda_{\alpha, t}\right)\right) \mathbf{i} \\
& =\sum_{\alpha \in \lambda} \sum_{t>r_{\alpha}} \varepsilon_{\alpha, t} v_{x}\left(\left[h_{\alpha}^{\prime}\right]_{z}\right) \operatorname{ch} S_{F}^{\lambda_{\alpha, t}},
\end{aligned}
$$

where the last equality follows by swapping the order of summation since, by definition, $\operatorname{ch} S_{F}^{\lambda_{\alpha}, t}=\sum_{\mathbf{i} \in I^{n}} d_{\mathbf{i}}\left(\lambda_{\alpha, t}\right) \mathbf{i}$. This completes the proof.

Remark 5.19 Using Lemma 4.6, if $h^{\prime} \in \mathbb{N}$ then we can explicitly compute $v_{x}\left(\left[h^{\prime}\right]_{z}\right)=$ $\sum_{f \mid h^{\prime}} v_{x}\left(\Phi_{f}(z)\right)$. In particular, ch $S_{F}^{\lambda_{\alpha, t}}$ appears in ch $J_{F}^{\lambda}$ only if $e$ divides $h_{\alpha}^{\prime}$. Further, in view of Proposition 5.11(d), if $1 \leq h_{\alpha}, h_{\alpha}^{\prime} \leq n$ then $\operatorname{ch} S_{F}^{\lambda_{\alpha, t}}$ appears in ch $J_{F}^{\lambda}$ if and only if $v_{x}\left(\left[h_{\alpha}\right]_{z}\right) \neq v_{x}\left(\left[h_{\alpha}^{\prime}\right]_{z}\right)$.

Acknowledgements We thank Jun Hu for useful discussions about the results of this paper and for his feedback. We thank the referee for their detailed comments. This research was supported, in part, by the Australian Research Council.

Funding Open Access funding enabled and organized by CAUL and its Member Institutions. 
Open Access This article is licensed under a Creative Commons Attribution 4.0 International License, which permits use, sharing, adaptation, distribution and reproduction in any medium or format, as long as you give appropriate credit to the original author(s) and the source, provide a link to the Creative Commons licence, and indicate if changes were made. The images or other third party material in this article are included in the article's Creative Commons licence, unless indicated otherwise in a credit line to the material. If material is not included in the article's Creative Commons licence and your intended use is not permitted by statutory regulation or exceeds the permitted use, you will need to obtain permission directly from the copyright holder. To view a copy of this licence, visit http://creativecommons.org/licenses/by/4.0/.

\section{References}

1. Ariki, S., Koike, K.: A Hecke algebra of $(\mathbf{Z} / r \mathbb{Z})$ ? $\mathfrak{S}_{n}$ and construction of its irreducible representations. Adv. Math. 106, 216-243 (1994)

2. Ariki, S., Mathas, A., Rui, H.: Cyclotomic Nazarov-Wenzl algebras, Nagoya Math. J., 182, 47-134 (2006). (Special issue in honour of George Lusztig), arXiv:math/0506467

3. Belinson, A., Bernstein, J.: A proof of Jantzen conjectures. In: I. M. Gel'fand Seminar, Adv. Soviet Math., 16, Amer. Math. Soc., Providence, RI, pp. 1-50 (1993)

4. Brundan, J.: Centers of degenerate cyclotomic Hecke algebras and parabolic category $\mathcal{O}$. Represent. Theory 12, 236-259 (2008)

5. Brundan, J., Kleshchev, A.: Graded decomposition numbers for cyclotomic Hecke algebras. Adv. Math. 222, 1883-1942 (2009)

6. Brundan, J., Kleshchev, A., Wang, W.: Graded Specht modules. J. Reine Angew. Math. 655, 61-87 (2011). arXiv:0901.0218

7. Brylinski, J.-L., Kashiwara, M.: Kazhdan-Lusztig conjecture and holonomic systems. Invent. Math. 64, 387-410 (1981)

8. Curtis, C.W., Reiner, I.: Methods of Representation Theory. Vol. II, Pure and Applied Mathematics (New York). With Applications to Finite Groups and Orders, A Wiley-Interscience Publication. Wiley, New York (1987) York). With Applications to Finite Groups and Orders, A Wiley-Interscience Publication. Wiley, New York (1987)

9. de Boeck, M., Evseev, A., Lyle, S., Speyer, L.: On bases of some simple modules of symmetric groups and Hecke algebras. Transform. Groups 23, 631-669 (2018)

10. Dipper, R., James, G., Mathas, A.: Cyclotomic $q$-Schur algebras. Math. Z. 229, 385-416 (1998)

11. Dipper, R., Mathas, A.: Morita equivalences of Ariki-Koike algebras. Math. Z. 240, 579-610 (2002)

12. Gabber, O., Joseph, A.: Towards the Kazhdan-Lusztig conjecture. Ann. Sci. École Norm. Sup. 14(4), 261-302 (1981)

13. Graham, J.J., Lehrer, G.I.: Cellular algebras. Invent. Math. 123, 1-34 (1996)

14. Hu, J., Mathas, A.: Graded cellular bases for the cyclotomic Khovanov-Lauda-Rouquier algebras of type A. Adv. Math. 225, 598-642 (2010). arXiv:0907.2985

15. Hu, J., Mathas, A.: Graded induction for Specht modules. Int. Math. Res. Not. IMRN 2012, 1230-1263 (2012). arXiv:1008.1462

16. Hu, J., Mathas, A.: Seminormal forms and cyclotomic quiver Hecke algebras of type A. Math. Ann. 364, 1189-1254 (2016). arXiv:1304.0906

17. James, G.: On the decomposition matrices of the symmetric groups. II. J. Algebra 43, 45-54 (1976)

18. James, G., Mathas, A.: A $q$-analogue of the Jantzen-Schaper theorem. Proc. Lond. Math. Soc. 74(3), 241-274 (1997)

19. James, G., Mathas, A.: The Jantzen sum formula for cyclotomic $q$-Schur algebras. Trans. Am. Math. Soc. 352, 5381-5404 (2000)

20. Jantzen, J.C.: Darstellungen halbeinfacher algebraischer Gruppen und zugeordnete kontravariante Formen. Bonn. Math. Schr. 67, v+124 (1973)

21. Jantzen, J.C.: Moduln mit einem höchsten Gewicht. Lecture Notes in Mathematics, vol. 750. Springer, Berlin (1979)

22. Jantzen, J.C.: Representations of Algebraic Groups, Mathematical Surveys and Monographs, vol. 107, 2nd edn. American Mathematical Society, Providence (2003)

23. Kang, S.-J., Kashiwara, M.: Categorification of highest weight modules via Khovanov-Lauda-Rouquier algebras. Invent. Math. 190, 699-742 (2012)

24. Kazhdan, D., Lusztig, G.: Representations of Coxeter groups and Hecke algebras. Invent. Math. 53, 165-184 (1979)

25. Kazhdan, D., Lusztig, G.: Affine Lie algebras and quantum groups. Int. Math. Res. Notices, 21-29 (1991) 
26. Khovanov, M., Lauda, A.D.: A diagrammatic approach to categorification of quantum groups. I. Represent. Theory 13, 309-347 (2009)

27. Kleshchev, A.: Linear and Projective Representations of Symmetric Groups, Cambridge Tracts in Mathematics, 163. Cambridge University Press, Cambridge (2005)

28. Lang, S.: Algebra, Graduate Texts in Mathematics, vol. 211, 3rd edn. Springer, New York (2002)

29. Lascoux, A., Leclerc, B., Thibon, J.-Y.: Hecke algebras at roots of unity and crystal bases of quantum affine algebras. Commun. Math. Phys. 181, 205-263 (1996)

30. Li, G.: Integral basis theorem of cyclotomic Khovanov-Lauda-Rouquier algebras of type A. J. Algebra 482, 1-101 (2017). arXiv:1412.3747

31. Littlewood, D.E.: Modular representations of symmetric groups. Proc. R. Soc. Lond. Ser. A. 209, 333-353 (1951)

32. Lusztig, G.: On quantum groups. J. Algebra 131, 466-475 (1990)

33. Mathas, A.: Iwahori-Hecke Algebras and Schur Algebras of the Symmetric Group, University Lecture Series, 15. American Mathematical Society, Providence (1999)

34. Mathas, A.: Matrix units and generic degrees for the Ariki-Koike algebras. J. Algebra 281, 695-730 (2004). arXiv:math/0108164

35. Mathas, A.: Seminormal forms and Gram determinants for cellular algebras. J. Reine Angew. Math., 619, 141-173 (2008). With an appendix by Marcos Soriano, arXiv:math/0604108

36. Mathas, A.: Cyclotomic quiver Hecke algebras of type A. In: G. W. Teck, K. M. Tan (eds) Modular Representation Theory of Finite and $p$-adic Groups. National University of Singapore Lecture Notes Series, 30, World Scientific, 2015, ch. 5, 165-266. arXiv:1310.2142

37. Mathas, A.: Restricting Specht modules of cyclotomic Hecke algebras. Sci. China Math., 61, 299-310 (2018). Special Issue on Representation Theory, arXiv:1610.09729

38. Rouquier, R.: Quiver Hecke algebras and 2-Lie algebras. Algebra Colloq. 19, 359-410 (2012)

39. Ryom-Hansen, S.: The Schaper formula and the Lascoux, Leclerc and Thibon algorithm. Lett. Math. Phys. 64, 213-219 (2003)

40. Schaper, K.-D.: Charakterformeln für Weyl-Moduln und Specht-Moduln in Primcharacteristik, diplomarbeit, Bonn (1981)

41. Shan, P.: Graded decomposition matrices of $v$-Schur algebras via Jantzen filtration. Represent. Theory 16, 212-269 (2012)

42. Soergel, W.: Andersen filtration and hard Lefschetz. Geom. Funct. Anal. 17, 2066-2089 (2008)

43. Williamson, G.: Local Hodge theory of Soergel bimodules. Acta Math. 217, 341-404 (2016)

44. Williamson, G.: Schubert calculus and torsion explosion. J. Am. Math. Soc. 30:1023-1046. With a joint appendix with Alex Kontorovich and Peter J, McNamara (2017). arXiv:1309.5055

45. Young, A.: On quantitative substitutional analysis I. Proc. Lond. Math. Soc. 33, 97-145 (1900)

46. Yvonne, X.: A conjecture for $q$-decomposition matrices of cyclotomic $v$-Schur algebras. J. Algebra 304, 419-456 (2006)

Publisher's Note Springer Nature remains neutral with regard to jurisdictional claims in published maps and institutional affiliations. 